\title{
Optimal interior and boundary regularity for almost minimizers to elliptic variational integrals
}

\author{
Frank DuzaAR* Klaus STefFen *
}

\begin{abstract}
We give a new proof of the small excess regularity theorems for integer multiplicity rectifiable currents of arbitrary dimension and codimension minimizing an elliptic parametric variational integral. This proof does not use indirect blow-up arguments, it covers interior and boundary regularity, it applies to almost minimizing currents, and it gives an explicit and often optimal modulus of continuity for the derivative, i.e. for the tangent plane field of the almost minimizing currents.
\end{abstract}

\section{Introduction}

The regularity theory for integer multiplicity rectifiable currents minimizing a parametric elliptic variational integral was initiated in the pioneering work of F.J. Almgren [Alm1], where the regularity is proved at interior support points with small cylindrical excess. Almgren's results are presented in F. Federer's monograph [Fe, Chap. 5]. E. Bombieri has given a somewhat simpler proof which also applies to approximately minimizing currents. This is important in order to be abble to include problems with side conditions, such as volume constraints or obstacle conditions, in the regularity theory (see Section 1). In [Alm2] Almgren also treated the more general situation of almost minimizing currents and sets. A new and elegant proof of Almgren's original regularity theorem was presented by R. Schoen and L. Simon [SS]. This proof avoids the indirect blow-up arguments of the previous authors and it uses a direct P.D.E. argument instead. The interior regularity theory for (almost) minimizing currents has been thus quite well developed for some years.

With regard to boundary regularity the situation is different. One only has the work of R. Hardt [Ha1] which showed how to extend the reasoning from [Fe, Chap. 5.3] to the boundary situation. Apart from [Ha1], we are not aware of any published treatment of boundary regularity for (almost) minimizing currents and general elliptic integrands. (This problem is addressed, however, in the unpublished thesis [Li] of F.H. Lin.)

Our present work comes from an attempt to understand the question of boundary regularity better and to give a simpler proof of Hardt's boundary regularity result, and if possible to extend it to the case of almost minimizing currents. We first tried to carry over the simple method of [SS] to the boundary situation. This failed, however, for reasons explained below. Instead we have combined arguments from [Bo] with the idea of harmonic approximation as presented in [Si1, 21.1] and we have added some new estimates related to the integrands one obtains from a given smooth integrand by transforming it with diffeomorphisms that flatten the boundary. As a result, we have obtained small excess regularity theorems for almost minimizing currents which give an optimal modulus of continuity for the tangent plane field in many situations. It seems that even in the interior situation such

${ }^{*}$ We are grateful to the Volkswagen-Stiftung and to the Oberwolfach Mathematical Research Institute whose support through the RiP collaborative research program made the commencement of this work possible. We also acknowledge the support of the Sonderforschungsbereich 288 Differential Geometry and Quantum Physics, Berlin. 
optimal regularity theorems are new and cannot be deduced from [Alm2], [Alm1], [Fe], [Bo], [SS] by simply giving quantitative versions of all the arguments involved.

We present our main result in Theorem 6.1 (the interior $\epsilon$-regularity theorem), and Theorem 6.4 (the optimal boundary $\epsilon$-regularity theorem). The precise technical assumptions are collected in Section 1 where we also explain the notion of almost minimizing currents. As an example for the boundary regularity statements one can deduce from Theorem 6.4 we state the following theorem:

0.1 Theorem (optimal boundary regularity). Suppose $F$ is a positive elliptic integrand of degree $n$ and of class $C^{2}$ on the open subset $D$ of $\mathbb{R}^{n+k}$ and $0<\beta<1$. Then there exists $\vartheta>0$ such that if $T$ is an integer multiplicity rectifiable $n$-current which is almost minimizing for $(F, \omega)$ in $D$ with modulus $\omega(r)=$ const $r^{2 \beta}$, if $a \in D \cap \operatorname{spt}(\partial T)$ and spt $(\partial T)$ locally at $a$ is an $(n-1)$-dimensional submanifold of class $C^{1, \beta}$ in $D$, and if the lower density of $\|T\|$ at a satisfies

$$
\Theta_{*}^{n}(\|T\|, a) \leq \frac{1}{2}+\vartheta
$$

then spt $T$ is locally at a an $n$-dimensional submanifold of class $C^{1, \beta}$ up to the boundary.

It can be seen from the proof in Section 7 that the constant $\vartheta>0$ depends only on the exponent $\beta$, the ellipticity constant of $F$, a bound for $F(x, \xi)$ and its derivatives up to second order with respect to the tangent $n$-plane variable $\xi$, and a modulus of continuity for the second derivative of $F$ with respect to $\xi$. Moreover, a version of Theorem 0.1 holds for boundaries $B$ satisfying a $C^{1, \kappa(\cdot)}$ Dini condition with modulus $\kappa(\cdot)$ such that $\mathrm{K}(r)=\int_{0}^{1} \varrho^{-1} \kappa(\varrho) d \varrho<\infty$, and for moduli of almost minimality $\omega(\cdot)$ such that $\Omega(r)=$ $\left[\int_{0}^{1} \varrho^{-1} \sqrt{\omega(\varrho)} d \varrho\right]^{2}<\infty$. Then spt $T$ is locally at $a$ an $n$-dimensional submanifold of Dini class $C^{1, \gamma(\cdot)}$ up to the boundary with modulus $\gamma(r)=$ const $\left[r^{2 \beta}+\mathrm{K}(r)^{2}+\Omega(r)\right]^{1 / 2}$ where $0<\beta<1$ can be prescribed. (The precise technical conditions on $\kappa(\cdot)$ and $\omega(\cdot)$ are described in Section 1.)

We should note that, in general, one cannot verify the density assumption for $\|T\|$ in Theorem 0.1 at least for some boundary points. In other words, it is not clear that the small excess boundary regularity theorem can be applied at all to minimizers of a given geometric variational problem. This unsatisfactory situation is in contrast to the interior theory where the $\epsilon$-regularity theorem can always be applied at a dense set of points in the support and, hence, the regular set of an almost minimizing current is open and dense in its support.

Of course, if one makes additional assumptions concerning the prescribed boundary or the integrand, then one can say more. For example, in [Ha1, Sec. 4] it is proved that one has boundary regularity of codimension one currents near boundary points satisfying an appropriate barrier condition. For the area integrand, R. Hardt and L. Simon [HS] have even proved full boundary regularity for minimizing currents of codimension one. (This has been extend to minimizing currents with prescribed mean curvature in [DS2].) The interior singular set of area minimizing codimension one currents is known to be of codimension at least 7, by Federer's result [Fe1] (see [Ma], [MM], [DS1] for currents of prescribed mean curvature). With regard to the area integrand we also mention [HaL], Allard's work [All1], [All2] on regularity and boundary regularity of minimizers in the varifold setting, and Almgren's monumental theory [Alm3] which gives interior regularity up to codimension 
two for area minimizing currents of arbitrary codimension. Finally, for codimension one minimizers of elliptic variational integrals we know that the singular set has codimension at least two, by the result of Schoen, Simon and Almgren [SSA]. In the present work, however, we are interested in general elliptic integrands and currents of arbitrary codimension, and we want to prove, starting from some weak initial assumptions (smallness of excess and a density condition) the best possible regularity for almost minimizing currents.

We now outline our proof of the regularity theorems. We begin in Section 2 with mass and height estimates for the rectifiable $n$-current $T$ in $\mathbb{R}^{n+k}$ which is almost minimizing with respect to an elliptic integrand $F$ and has small excess over the $n$-plane $\mathbb{R}^{n} \times\{0\}^{k}$. These estimates are standard and extended to the boundary situation, where $\partial T$ has support in $\mathbb{R}^{n-1} \times\{0\}^{k+1}$, without difficulties. The next step is to approximate $T$ by the graph of a Lipschitz function $g$. The Lipschitz approximation is also well-known in the interior situation and easily established in the boundary case. We present in Section 3 a suitable version. Since $T$ is almost minimizing for $F$ it can be shown, along the lines of [Bo], that $g$ is an approximate solution (in a certain sense) to the homogeneous system of P.D.E. associated with the constant coefficient elliptic quadratic form $A$ that is obtained from $F$ by freezing the coefficients at a point. We will call exact solutions of this system $A$-harmonic functions in what follows.

It is known from Almgren's work [Alm1] that the crucial step in the regularity proof is excess improvement, i.e. one must find a new $n$-plane $\tilde{S}$, obtained from $S=\mathbb{R}^{n} \times\{0\}^{k}$ by an isometry close to the identity, such that the excess of $T$ over this tilted $n$-plane $\tilde{S}$ is substantially smaller than the excess of $T$ over $S$. In Section 5 we establish such an excess improvement (Lemma 5.2) and derive from it a growth condition for the cylindrical excess as a function of the radius of the cylinder (Lemma 5.4). From this excess growth condition the $\epsilon$-regularity theorems of Section 6 then follow in a well-known manner, e.g. as in [Ha1], but we give full proofs in order to obtain an explicit modulus of continuity for the tangent plane field of $T$.

The essential point in which our proof differs from the previous ones, is the method of finding the tilted $n$-plane $\tilde{S}$ with improved excess. In Almgren's and Bombieri's work [Alm1], [Alm2], [Bo], [Fe, 5.3] this is done using blow-up by a sequence of certain scaling transformations $\sigma_{\nu}$ for which the scaled Lipschitz approximations $g_{\nu}$ converge to an $A$ harmonic function $u$. The plane $\tilde{S}$ is then obtained from the tangent plane to graph $u$ (at the appropriate point) by the inverse scaling $\sigma_{\nu}^{-1}$ with $\nu \gg 1$. This proof of the excess improvement lemma is indirect and, hence, does not give an explicit constant in the estimate.

The idea of Schoen and Simon [SS] is to choose $u$ as a solution of the Dirichlet problem associated with $A$ on a ball, taking the values of the Lipschitz approximation $g$ as boundary values. The plane $\tilde{S}$ is then chosen as the tangent plane to graph $u$ at the center of the ball. However, one needs a $C^{1, \delta}$ estimate for $u$ up to the boundary of the ball in order to prove that the excess of $T$ over $\tilde{S}$ is substantially smaller, and therefore $g$ has to be replaced by a smoothing $g_{\delta}$ of $g$ with appropriately chosen smoothing radius (depending on the size of the excess over the original plane $S$ ). It is this point which creates problems when treating the boundary regularity problem. There one would have to consider half balls, and we could not find a way to smooth the half balls and the Lipschitz function $g$ in such a way that the crucial estimates could be obtained along the lines of [SS]. Furthermore, as Schoen and Simon employ the Euler equation for the minimizing current $T$, it is not immediately clear 
how to treat almost minimizing currents.

Our method here is to replace the Lipschitz function $g$, which is already known to be approximatively $A$-harmonic, by a nearest $A$-harmonic function, in a sense. This is made possible by a simple lemma (Lemma 3.3) analogous to [Si2, 21.1]. In order to show that the tangent plane $\tilde{S}$ to the graph of this function $u$ gives the desired excess improvement we need a reverse Poincaré inequality (Caccioppoli inequality). We establish this in Lemma 4.1 in a weak form, which can be proved in the boundary situation as in [Bo], and we use a covering argument from [Si2] to derive from this the required strong version (Lemma 4.2).

Of course, all the ingredients of our proof have already been used previously in regularity theory. In particular, the idea of approximating (almost) minimizing currents by the solution of a constant coefficient elliptic P.D.E. system is by now a standard technique, dating back to DeGiorgi [DeG]. We believe, however, that the way in which we have combined the ingredients in our proof is new and has definite conceptual advantages: It is the first unified treatment of interior and boundary regularity; it applies to almost minimizing currents; it does not use an indirect blow-up argument and gives constants which are (in principle) explicitely computable in the regularity estimates; it enables one to derive an explicit and frequently optimal modulus of continuity for the derivative (i.e. the tangent plane field); it uses only natural and quite weak assumptions on the elliptic integrand; it is very flexible and can be applied to regularity questions related to (almost) minimizers of variational integrals or to weak solutions of nonlinear elliptic P.D.E. systems in a variety of different situations. This last point is perhaps the most important one. In fact, variants of the method used in this paper play a role in [DGr], [DGa], and [DGG] to obtain new and sometimes optimal regularity results.

Finally, we want to point out that the fact that our proof only requires minimal assumptions on the integrand $F$ is not merely an irrelevant technicality, but essential for optimal boundary regularity. Namely, if we study boundary regularity of an (almost) minimizing current $T$ for a smooth elliptic integrand $F$, and we have small excess and a prescribed boundary of class $C^{1, \beta}$, say, then an almost unavoidable procedure is to flatten the boundary by transforming it into an affine subspace with the same dimension using suitable $C^{1, \beta}$ diffeomorphisms. This will give correspondingly transformed currents $\tilde{T}$ and integrands $\tilde{F}$ such that $\tilde{T}$ is almost minimizing with respect to $\tilde{F}$ and $\tilde{T}$ has a flat boundary. However, $\tilde{F}(x, \xi)$ will only be $C^{1, \beta}$ with respect to the space variable $x$, and therefore we have to prove $C^{1, \beta}$ regularity for minimizers to integrands with this weak regularity in the case of a flat boundary in order to derive the expected $C^{1, \beta}$ regularity of $T$ up to the boundary. In fact, with a straightforward adaptation of the interior regularity theory to the case of a flat boundary, with the given $C^{1, \beta}$ smoothness of $\tilde{F}$, we could only establish $C^{1, \beta /(2-\beta)}$ regularity of $\tilde{T}$ losing something in the Hölder exponent. (We do not know whether this loss, which also occurs in a variety of other situations, is merely a technical problem or is indeed unavoidable.) In our estimates therefore we needed to keep distinct the continuity modulus of $x \mapsto \tilde{F}(x, \xi)$ from the continuity modulus of the same function in directions perpendicular to the flat boundary. For $\tilde{F}$ as above, this second modulus is substantially smaller than the first one. Carefully tracking how the different moduli enter the various estimates (which is possible in our regularity proof), we were able to prove in the end $C^{1, \beta}$ regularity of $T$ up to the boundary without any loss in the Hölder exponent. This way of proceeding is a new technique introduced in the present paper that may also be of interest with regard to other boundary regularity problems. 


\section{Rectifiable currents, parametric integrands, and almost mini- mizers}

By an $n$-dimensional integer multiplicity rectifiable current $T$ on $\mathbb{R}^{n+k}$ we mean a generalized oriented surface which can be represented by integration as a linear functional on the space of smooth $n$-forms $\alpha$ with compact support in $\mathbb{R}^{n+k}$ as follows

$$
\langle T, \alpha\rangle=\int_{M_{T}}\langle\alpha, \vec{T}\rangle \vartheta_{T} d \mathcal{H}^{n}=\int_{\mathbb{R}^{n+k}}\langle\alpha, \vec{T}\rangle d\|T\|
$$

Here $\mathcal{H}^{n}$ denotes the $n$-dimensional Hausdorff measure in $\mathbb{R}^{n+k}$, the supporting set $M_{T} \subset$ $\mathbb{R}^{n+k}$ is countably $\left(\mathcal{H}^{n}, n\right)$ rectfiable (i.e. it can be covered by countably many $n$-submanifolds of class $C^{1}$ in $\mathbb{R}^{n+k}$ and a set of zero $\mathcal{H}^{n}$ measure), $\vartheta_{T}$ is a positive integer valued $\mathcal{H}^{n}$ summable multiplicity function, and $\vec{T}$ is an $n$-measurable orientation $\boldsymbol{n}$-vectorfield for $M_{T}$ (i.e. $\vec{T}(x)$ equals, at $\mathcal{H}^{n}$ almost every point $x \in M_{T}$, the exterior product of some orthonormal basis in the $n$-dimensional approximate tangent space $\operatorname{Tan}^{n}\left(\mathcal{H}^{n}\left\llcorner M_{T}, x\right)\right)$. As usual, we have written $\|T\|$ for the Radon measure $\mathcal{H}^{n}\left\llcorner\vartheta_{T}\right.$ associated with $T$, understanding that $\vartheta_{T}$ is extended by the value zero on $\mathbb{R}^{n+k} \backslash M_{T}$, and we note that summability of $\vartheta_{T}$ is equivalent to finiteness of the mass (or $n$-area)

$$
\mathbf{M}(T)=\|T\|\left(\mathbb{R}^{n+k}\right)=\int_{M_{T}} \vartheta_{T} d \mathcal{H}^{n}
$$

We refer to [Fe] and [Si1] for the basic notions and notations related to currents. Notations not explained in this paper are taken from [Fe]. Our usage is consistent with this reference, except that what we have called an integer multiplicity rectifiable current above would be a locally rectifiable current with finite mass in the language of [Fe]. The abelian group of these $n$-dimensional currents on $\mathbb{R}^{n+k}$ is denoted by $\mathcal{R}_{n}\left(\mathbb{R}^{n+k}\right)$, its elements will also be called rectifiable $n$-currents or simply $n$-currents. We will always assume dimension $n \geq 2$ and codimension $k \geq 1$.

A parametric integrand of degree $n$ on an open set $D$ in $\mathbb{R}^{n+k}$ is a continuous map

$$
F: D \times \bigwedge_{n} \mathbb{R}^{n+k} \rightarrow \mathbb{R}
$$

which is positively homogeneous of degree one in the second variable, i.e. $F(x, r \xi)=r F(x, \xi)$ for $r>0$. The integral of $F$ over an $n$-current as above (with $\vartheta_{T} \equiv 0$ outside $D$ ) is then

$$
\mathbf{F}(T)=\int_{M_{T}} F(x, \vec{T}(x)) \vartheta_{T} d \mathcal{H}^{n} x=\int_{D} F(x, \vec{T}(x)) d\|T\| x
$$

provided the integral exists and is finite. This is the case, for example, if $|F|$ is bounded by the area integrand, i.e. $|F(x, \xi)| \leq \Lambda|\xi|$ holds for all $(x, \xi)$ with a finite constant $\Lambda$. For the purpose of regularity theory it is no restriction to assume $D=\mathbb{R}^{n+k}$.

We now list the assumptions on the integrand which we shall use in our regularity theorems. The first set of assumptions requires bounds for $F(x, \xi)$ and its derivatives $D_{(2)} F$, $D_{(2)}^{2} F$ with respect to the tangent plane variable $\xi \in \bigwedge_{n} \mathbb{R}^{n+k}$ as follws:

$$
|\xi| \leq F(x, \xi) \leq \Lambda|\xi|
$$




$$
\left\|D_{(2)} F(x, \xi)\right\| \leq \Lambda
$$

$$
\left\|D_{(2)}^{2} F(x, \xi)\right\| \leq \Lambda|\xi|^{-1}
$$

for all $x \in \mathbb{R}^{n+k}$ and $0 \neq \xi \in \bigwedge_{n} \mathbb{R}^{n+k}$, where $\Lambda \geq 1$ is a finite constant. (1.1) means, in particular, that $F$ is estimated from above and from below by the area integrand $M(x, \xi)=|\xi|$. (It is convenient to assume the constant 1 in the estimate from below; this is, of course, no essential restriction.) We also need a uniform modulus of continuity for $\xi \mapsto D_{(2)}^{2} F(x, \xi)$,

$$
\left\|D_{(2)}^{2} F(x, \xi)-D_{(2)}^{2} F(x, \tilde{\xi})\right\| \leq \nu(|\xi-\tilde{\xi}|)
$$

for all $x \in \mathbb{R}^{n+k}$ and $\xi, \tilde{\xi} \in \bigwedge_{n} \mathbb{R}^{n+k}$ with $|\xi|=1=|\tilde{\xi}|$, where $\nu(0+)=0=\nu(0)$. We assume continuity of $F$ and $D_{(2)} F$ with respect to the space variable $x$ as follows:

$$
\begin{aligned}
& |F(x, \xi)-F(\tilde{x}, \xi)| \leq \kappa(|x-\tilde{x}|)|\xi| \\
& \left\|D_{(2)} F(x, \xi)-D_{(2)} F(\tilde{x}, \xi)\right\| \leq \kappa(|x-\tilde{x}|)
\end{aligned}
$$

for all $x, \tilde{x}, \xi$, where $\kappa:[0, \infty[\rightarrow[0, \infty]$ is nondecreasing with $\kappa(0+)=0=\kappa(0)$ and ] $0, \infty\left[\ni r \mapsto \frac{1}{r} \kappa(r)\right.$ nonincreasing. We may also assume that $\kappa(1)$ is as small as we like; this can be achieved by scaling with homotheties. In the proof of the regularity theorem we need a restriction on the decay of $\kappa(r)$ as $r \downarrow 0$, cf. (1.15). This condition is satisfied when $\kappa(r) \leq r^{\beta}$ holds for some $\beta>0$ and all sufficiently small $r>0$. We also have to require that $r \mapsto r^{-\alpha} \kappa(r)$ is nonincreasing for some exponent $0<\alpha<1$.

In (1.1)-(1.6) we assume implicitely that all the appearing derivatives of $F$ depend continuously on $(x, \xi), \xi \neq 0$. With regard to the notation of norms we use $|\cdot|$ to denote natural Euclidean norms and $\|\cdot\|$ for operator type norms (largest Euclidean norm of values obtaind from evaluating on unit vectors).

For the treatement of boundary regularity we also need the continuity modulus $\mu(\bullet)$ of $x \mapsto F(x, \xi)$ in directions perpendicular to the boundary tangent plane $\mathbb{R}^{n-1} \times\{0\}$, i.e.

$$
\begin{aligned}
& |F(x, \xi)-F(\tilde{x}, \xi)| \leq \mu(|x-\tilde{x}|)|\xi|, \quad \text { if } x_{i}=\tilde{x}_{i} \text { for } i=1, \ldots, n-1, \\
& \left\|D_{(2)} F(x, \xi)-D_{(2)} F(\tilde{x}, \xi)\right\| \leq \mu(|x-\tilde{x}|), \quad \text { if } x_{i}=\tilde{x}_{i} \text { for } i=1 \ldots, n-1,
\end{aligned}
$$

where $0 \leq \mu(r) \leq \kappa(r)$ is a nondecreasing function of $r \geq 0$ with $] 0, \infty\left[\ni r \mapsto \frac{1}{r} \mu(r)\right.$ nondecreasing and a further condition stated in (1.15) below. The reason for introducing the modulus $\mu(\cdot)$ is the following: If $F$ is obtained from a smooth integrand by transformation with a diffeomorphism $\Phi$ that maps a given $C^{1}$ submanifold, of $\mathbb{R}^{n+k}$ to its tangent plane $\mathbb{R}^{n-1} \times\{0\} \subset \mathbb{R}^{n+k}$ at the origin and has the form $\Phi(x)=x+\Psi\left(x_{1}, \ldots, x_{n-1}\right)$, then the modulus $\kappa(\cdot)$ of $F$ is essentially the continuity modulus of the tangent plane distribution of , while $\mu(r) \leq$ const $r$ is a Lipschitz modul even when, is not of class $C^{1,1}$. We shall exploit this fact to obtain optimal moduli of continuity in the boundary regularity theorem. 
With $\kappa(\cdot)$ and $\mu(\cdot)$ we associate the following functions $\widehat{\kappa}(\cdot)$ and $\widehat{\mu}(\cdot)$ which enter the regularity estimates:

$$
\widehat{\kappa}(r)=\sup _{0 \leq 2 s \leq 1}\left[\kappa(s)-\left(\frac{s}{r}\right)^{2}\right] \quad \text { and } \quad \widehat{\mu}(r)=\sup _{0 \leq 2 s \leq 1}\left[\mu(s)-\left(\frac{s}{r}\right)^{2}\right] \quad \text { for } r>0 .
$$

One readily verifies that $\widehat{\mu}(\cdot)$ is nondecreasing with $\widehat{\mu}(0+)=0$ and $\widehat{\mu}\left(\frac{1}{2}\right) \leq \mu\left(\frac{1}{2}\right) \leq \frac{1}{4}+\widehat{\mu}(1)$. Furthermore, from the fact that $\mu\left(\vartheta^{2} r\right) \geq \vartheta^{2} \mu(r)$ holds for $0<\vartheta \leq 1$ we deduce $\widehat{\mu}(\vartheta r) \geq$ $\vartheta^{2} \widehat{\mu}(r)$ and, hence, that $r \mapsto \frac{1}{r} \sqrt{\widehat{\mu}(r)}$ is nonincreasing. We also note that $\frac{1}{4} \mu(r)^{2} \leq \widehat{\mu}(r) \leq$ $\mu(r)$ holds for $0<r \leq r_{0}$ whenever $0<r_{0} \leq \frac{1}{2}$ and $\mu\left(r_{0}\right) \leq 1$. The first inequality follows by taking $s=\frac{1}{\sqrt{2}} r \mu\left(\frac{1}{\sqrt{2}} r\right)$ in (1.9) and using $\mu(s) \geq \frac{1}{\sqrt{2}} \mu\left(\frac{1}{\sqrt{2}} r\right)^{2} \geq \frac{1}{2} \mu(r)^{2}$, by the nonincreasing property of $r \mapsto \frac{1}{r} \mu(r)$. For the second inequality one observes that $\mu(s)-\left(\frac{s}{r}\right)^{2}$ is nonpositive for $r \leq s \leq \frac{1}{2}$ and uses again (1.9) together with $\mu(s) \leq \frac{s}{r} \mu(r)$ for $0<s \leq r$. As an example, we have $\widehat{\mu}(r)=$ const $c^{\frac{2}{2-\beta}} r^{\frac{2 \beta}{2-\beta}}$ for $0<r \leq 1$, if $\mu(s)=c s^{\beta}$ with $0<\beta \leq 1$ and $0 \leq c \leq 2^{\beta-2}$. The same statements hold for $\kappa$ and $\widehat{\kappa}$, of course.

When dealing with the nonparametric situation where the $n$-currents are represented by graphs in $\mathbb{R}^{n} \times \mathbb{R}^{k}$ we will have to consider the associated nonparametric integrand

$$
F^{\S}: \mathbb{R}^{n} \times \mathbb{R}^{k} \times \operatorname{Hom}\left(\mathbb{R}^{n}, \mathbb{R}^{k}\right) \rightarrow \mathbb{R}
$$

which is characterized by

$$
\mathbf{F}\left(T_{g}\right)=\int_{\Omega} F^{\S}(y, g(y), D g(y)) d \mathcal{L}^{n} y
$$

whenever $g: \Omega \rightarrow \mathbb{R}^{k}$ is Lipschitz on a bounded domain $\Omega$ in $\mathbb{R}^{n}$ and $T_{g}$ is the $n$-current representing the graph of $g$ with natural orientation and multiplicity one. We have

$$
F^{\S}(y, z, p)=F\left((y, z), \bigwedge_{n}(\mathrm{id}, p) e_{1} \wedge \ldots \wedge e_{n}\right)
$$

where $e_{1}, \ldots, e_{n}$ is the canonical basis in $\mathbb{R}^{n}$ and $(\mathrm{id}, p)(y)=(y, p y)$ for $y \in \mathbb{R}^{n}, p \in$ $\operatorname{Hom}\left(\mathbb{R}^{n}, \mathbb{R}^{k}\right)$. It is convenient to assume conditions analogous to (1.3), (1.4) also for the total second order derivative $D_{(3)}^{2} F^{\S}$ of $F^{\S}$ with respect to $p$, i.e.

$$
\begin{gathered}
\left\|D_{(3)}^{2} F^{\S}(y, z, p)\right\| \leq \Lambda \text { for all } y, z, p \text { with }|p| \leq 1 \\
\left\|D_{(3)}^{2} F^{\S}(y, z, p)-D_{(3)}^{2} F^{\S}(y, z, \tilde{p})\right\| \leq \nu(|p-\tilde{p}|) \\
\quad \text { for all } y, z, p, \tilde{p} \text { with }|p| \leq 2,|\tilde{p}| \leq 2
\end{gathered}
$$

Actually these conditions follow from (1.1)-(1.4) with suitably modified constant $\Lambda$ and modulus $\nu$. For $\nu$ we will assume, as we may, that it is a nondecreasing function with $\nu(0+)=0=\nu(0)$.

We next come to the assumption of ellipticity for $F$. This is a condition on the constant coefficient integrands $F_{a}(x, \xi)=F(a, \xi)$ obtained from $F$ by freezing the coefficients at $a \in \mathbb{R}^{n+k}$. Following [Fe, 5.1.2] we call $F$ elliptic at $a$, if there exists a positive constant $\lambda$ such that the inequality

$$
\mathbf{F}_{a}(T)-\mathbf{F}_{a}(S) \geq \lambda[\mathbf{M}(T)-\mathbf{M}(S)]
$$


holds whenever $S, T$ are (compactly supported) rectifiable $n$-currents on $\mathbb{R}^{n+k}$ with the same boundary $\partial S=\partial T$ and $S$ is represented by an $\mathcal{H}^{n}$ measurable subset of some $n$-dimensional affine subspace in $\mathbb{R}^{n+k}$ with constant orientation $n$-vectorfield and $\mathcal{H}^{n}$ summable positive integer valued multiplicity. (This is the ellipticity condition convenient for us. In Almgren's original definition [Alm1] only flat $n$-disks were considered. The ellipticity definition in [Bo] is an adaptation of this taking multiplicities into account; it assumes (1.12) only when $S$ is an integer multiple of a flat $n$-disc with constant orientation. It can be shown that this is equivalent to the definition above.) We will suppose that $F$ is uniformly elliptic in the sense that (1.12) holds with the same constant $\lambda$ for all points $a \in \mathbb{R}^{n+k}$. This is no restriction as our considerations are of local nature. For the ellipticity constant we assume $0<\lambda \leq 1$.

If $F$ is elliptic at $a=(b, c) \in \mathbb{R}^{n} \times \mathbb{R}^{k}$, then the associated nonparametric integrand satisfies the Legendre-Hadamard condition in the sense that the Hessean of $F^{\S}(b, c, p)$ with respect to the variable $p=\left(p_{\alpha}^{i}\right) \in \operatorname{Hom}\left(\mathbb{R}^{n}, \mathbb{R}^{k}\right)$ is rank one elliptic. In particular, if (1.12) holds, then with the same constant $\lambda$ we have ([Fe, 5.1.10])

$$
\sum_{\alpha, \beta=1}^{n} \sum_{i, j=1}^{k} \frac{\partial^{2} F^{\S}}{\partial p_{\alpha}^{i} \partial p_{\beta}^{j}}(b, c, 0) \eta_{\alpha} \zeta^{i} \eta_{\beta} \zeta^{j} \geq \lambda|\eta|^{2}|\zeta|^{2}
$$

for all $\zeta$ in $\mathbb{R}^{k}$ and $\eta$ in (the dual space of) $\mathbb{R}^{n}$. The nonparametric Legendre-condition does not imply, however, ellipticity (1.12) if $n \geq 2, k \geq 2$ (see [Sv2]). On the other hand, the parametric Legendre condition [Fe, 5.1.3], which was adopted in [SS] as the definition of ellipticity, is presumably more restrictive than (1.12) if $n \geq 2, k \geq 2$. This is suggested by the analogous result for nonparametric variational integrals (e.g. [Sv1]).

Recalling [Fe, 5.1.1] for the transformation of integrands $F$ by diffeomorphisms $\Phi$ and [Fe, 5.1.4] for the preservation of ellipticity under such transformations one readily verifies that the integrand $\tilde{F}$, obtained from the transformed integrand $\Phi^{\#} F$ by multiplying with a suitable positive factor and scaling with a suitable homothety of $\mathbb{R}^{n+k}$, satisfies the same set of conditions (1.1)-(1.13) with appropriate constants $\tilde{\Lambda}, \tilde{\lambda}$ and moduli $\tilde{\kappa}$, $\tilde{\nu}$, provided $\Phi$ is a biLipschitz diffeomorphism of $\mathbb{R}^{n+k}$ with $D^{2} \Phi$ bounded or $D \Phi$ Lipschitz, at least. In this sense our assumptions on the integrand are invariant with respect to biLipschitz diffeomorphisms of class $C^{1,1}$.

A rectifiable $n$-current $T$ is called $F$ minimizing in an open subset $D$ of $\mathbb{R}^{n+k}$ if $\mathbf{F}(T) \leq$ $\mathbf{F}(T+X)$ for all closed $n$-currents $X$ with compact support in $D$. Following Almgren [Alm2] and Bombieri [Bo] we consider a relaxed minimizing condition which allows the existence of modifications $T+X$ of $T$ with $\mathbf{F}(T+X)$ smaller than $\mathbf{F}(T)$, but only by a factor $1+\omega(r)$ close to 1 if $X$ has its support in a ball of small radius $r$. We adopt the definition from [Bo] which is as follows: For a given function $\omega:] 0, \infty[\rightarrow[0, \infty]$ one says that $T$ is almost minimizing for $(F, \omega)$ in $D$, or $(F, \omega)$ minimizing for short, if

$$
\mathbf{F}(T) \leq \mathbf{F}(T+X)+\omega(r) \mathbf{M}(T\llcorner K+X)
$$

holds for all closed $n$-currents $X$ with support in a compact set $K \subset D$ which is contained in a ball of radius $r$ in $\mathbb{R}^{n+k}$.

With regard to $\omega$ we will always assume that it is a nondecreasing function and finite for the values of $r$ appearing in the following. For the regularity theory we need (1.14) only for small radii $r$, of course, i.e. we can admit $\omega(r)=\infty$ for $r \geq \delta$ and some $\delta>0$. 
Furthermore, it would be sufficient to know (1.14) for all $X$ satisfying an additional mass bound $\mathbf{M}(X) \leq \gamma$ with some constant $0<\gamma<\infty$. Additional assumptions on $\omega$ and on the moduli $\kappa, \widehat{\kappa}, \mu, \widehat{\mu}$ defined previously are needed for the iteration argument in the proof of the excess decay, Lemma 5.4. There we assume that $r^{-\alpha} \kappa(r), r^{-\alpha} \mu(r), r^{-2 \alpha} \omega(r)$ are nonincreasing functions of $r>0$ for some exponent $0<\alpha<1$ (this is not an essential restriction, but it excludes Lipschitz modules $\kappa(r)=$ const $r$; as before, it follows that $r \mapsto r^{-2 \alpha} \widehat{\kappa}(r)$ and $r \mapsto r^{-2 \alpha} \widehat{\mu}(r)$ are also nonincreasing) and we require that

$$
\begin{array}{cc}
\mathrm{K}(r)=\int_{0}^{r} \frac{1}{\varrho} \kappa(\varrho) d \varrho<\infty, & \widehat{\mathrm{K}}(r)=\left[\int_{0}^{r} \frac{1}{\varrho} \sqrt{\widehat{\kappa}(\varrho)} d \varrho\right]^{2}<\infty, \\
\widehat{\mathrm{M}}(r)=\left[\int_{0}^{r} \frac{1}{\varrho} \sqrt{\widehat{\mu}(\varrho)} d \varrho\right]^{2}<\infty, & \Omega(r)=\left[\int_{0}^{r} \frac{1}{\varrho} \sqrt{\omega(\varrho)} d \varrho\right]^{2}<\infty
\end{array}
$$

for small $r>0$. This growth condition for $\omega(r)$ as $r \downarrow 0$ appears already in [Alm2], [Bo], [Ta1] and it is, in a sense, necessary for $C^{1}$ regularity of $(F, \omega)$ minimizers as shown by examples in [Ta1] (for the area integrand with codimension $k=1$ and a somewhat different notion of almost minimality). Note that $\omega(r)=r^{2 \beta}$ and $\kappa(r)=r^{\beta}$ with $0<\beta<1$ satisfies all the assumptions above with $\Omega(r)=\beta^{-2} r^{2 \beta}$ and $\mathrm{K}(r)=\beta^{-1} r^{\beta}$. In view of $\widehat{\mu}(r) \leq \mu(r)$ for sufficiently small $r>0$ the condition $\widehat{\mathrm{M}}(r)<\infty$ is satisfied for small $r>0$ whenever $\int_{0}^{r} \frac{1}{\varrho} \sqrt{\mu(\varrho)} d \varrho<\infty$ holds, so that $\mu(r)=r^{\beta}$ with $0<\beta<1$ is again admissible here.

We now discuss some situations were $(F, \omega)$ minimizing currents arise. The first examples are minimizers for functionals of mean curvature type. Here we assume that the $n$-current $T$ is minimizing for $\mathbf{F}+\mathbf{V}$ on $\mathbb{R}^{n+k}$ where $\mathbf{V}$ is some kind of additive volume functional. More precisely, suppose $|\mathbf{V}(X)| \leq \gamma \mathbf{M}(Q)$ whenever $X$ is a closed rectifiable $n$-current and $Q$ an $(n+1)$-current of least mass with $\partial Q=X$. Then we have

$$
\mathbf{F}(T) \leq \mathbf{F}(T+X)+\mathbf{V}(X) \leq \mathbf{F}(T+X)+\gamma \mathbf{M}(Q)
$$

and if spt $X \subset K$, where $K$ is contained in a ball of radius $r$, we can estimate $\mathbf{M}(Q)$ by the mass of the cone over $X$, to obtain

$$
\mathbf{M}(Q) \leq \frac{r}{n+1} \mathbf{M}(X) \leq \frac{r}{n+1}[\mathbf{M}(T\llcorner K+X)+\mathbf{M}(T\llcorner K)]
$$

With the help of (1.1) we readily deduce from (1.16), (1.17) that $T$ is $(F, \omega)$ minimizing with a function $\omega(r)=O(r)$ as $r \downarrow 0$. Functionals of the type $\mathbf{M}+\mathbf{V}$ occur as energies in the variational approach to hypersurfaces with prescribed mean curvature or surfaces with prescribed mean curvature vector (e.g. [Du1], [DF1], [DF2], [DS3], [Ma]). In [DS1] currents with the almost minimizing property (1.16) for the area integrand $F=M$ were systematically studied.

A similar situation occurs when $T$ minimizes $\mathbf{F}$ subject to a volume constraint $\mathbf{V}(T)=$ const. If the first variation of $\mathbf{V}$ does not vanish and $X, K, r$ are as above with $r$ sufficiently small and $\mathbf{M}(X)$ bounded by a finite constant, then we can correct the volume of $T+X$ by a deformation $h_{t}$ which is the identity on $K$ to achieve $\mathbf{V}\left(h_{t \#} T+X\right)=\mathbf{V}(T)$ and $\left|\mathbf{F}\left(h_{t \#} T+X\right)-\mathbf{F}(T+X)\right| \leq \mathrm{const}|\mathbf{V}(X)|$. Since $h_{t \#} T+X$ satisfies the volume constraint we have $\mathbf{F}(T) \leq \mathbf{F}\left(h_{t \#} T+X\right)$, and with $|\mathbf{V}(X)| \leq \gamma \mathbf{M}(Q)$ and (1.17) we see as before that $T$ is $(F, \omega)$ minimizing (for the unconstrained problem) with $\omega(r)=O(r)$ as $r \downarrow 0$. 
See [Du2], [GMT], [Ta3] for a more thorough discussion in the case $F=M$ and $k=1$ and [Alm2] for general $F$ and $k=1$.

If $T$ minimizes $F$ subject to an obstacle condition spt $T \subset A$, where $A \subset \mathbb{R}^{n+k}$ is closed and $\mathbb{R}^{n+k} \backslash A$ is the "obstacle", then one can derive $(F, \omega)$ minimality of $T$ (for the unconstrained problem), provided $A$ is sufficiently regular at the boundary and the integrand $F$ is compatible with the obstacle. By the latter condition we mean that for $0<r$ sufficiently small and each point $a \in \partial A$ there is a Lipschitz retraction $\pi_{a}$ of the ball $\mathbf{U}(a, r)$ in $\mathbb{R}^{n+k}$ onto $A \cap \mathbf{U}(a, r)$ such that $\mathbf{F}_{a}\left(\pi_{a \#} S\right) \leq \mathbf{F}_{a}(S)$ holds for $n$-currents $S$ with spt $S \subset \mathbf{U}(a, r)$ and spt $\partial S \subset A \cap \mathbf{U}(a, r)$. If $X$ is closed with support in $\mathbf{U}(a, r)$, then we can use $\pi_{a \#}(T\llcorner\mathbf{U}(a, r)+X)$ as a comparison current for $T\llcorner\mathbf{U}(a, r)$, and with the help of (1.5) we deduce that $T$ is $(F, \omega)$ minimizing with $\omega(r)=O(r)$ as $r \downarrow 0$ (assuming that the conditions on the obstacle hold uniformly for all $a \in \partial A$ ). More generally we assume that $\pi_{a}$ retracts $\mathbf{U}(a, r)$ without decreasing $\mathbf{F}_{a}$ onto a subset of $\mathbf{U}(a, r)$ which is diffeomorphic to $A \cap \mathbf{U}(a, r)$ by a $C^{1}$ diffeomorphism $\varphi$ of $\mathbf{U}(a, r)$ that deviates in the $C^{1}$ norm from the identity by at most const $r^{\beta}, 0<\beta \leq 1$. Using then $\left(\varphi \circ \pi_{a} \circ \varphi^{-1}\right)_{\#}(T+X)\llcorner\mathbf{U}(a, r)$ as comparison current for $T\llcorner\mathbf{U}(a, r)$ in the obstacle problem we obtain the $(F, \omega)$-minimizing property for $T$ with $\omega(r)=O\left(r^{\beta}\right)$ as $r \downarrow 0$. This can be applied, for example, if $F=M$ is the area integrand and $A$ is the closure of a (uniform) $C^{1, \beta}$ domain with $\pi_{a}$ the nearest point retraction onto the affine tangent half space to $A$ at $a$. (Actually $\omega(r)=O\left(r^{2 \beta}\right)$ in this case by the argument below.) More generally we may take $A$ locally uniformly $C^{1, \beta}$ equivalent, in a suitable sense, to a convex set. For general elliptic integrands $F$, codimension $k=1$, and $A$ the closure of a uniform $C^{1, \beta}$ domain we can again use the nearest point retraction $\pi_{a}$ onto the tangent half space of $A$ at $a$, as the $\mathbf{F}_{a}$ nonincreasing property of $\pi_{a \#}$ is then a consequence of ellipticity. Thus, solutions of obstacle problems will be $(F, \omega)$ minimizing with $\omega(r)=O\left(r^{\beta}\right)$. We refer to [Alm2], [Li], and (for the area integrand with codimension 1 and multiplicity 1$)$ to [Ta1], [Mi].

Finally, we want to indicate that every oriented uniform $C^{1, \beta}$ submanifold $\mathcal{T}$ of dimension $n$ in $\mathbb{R}^{n+k}$ is $(M, \omega)$ minimizing with $M$ the area integrand and $\omega(r)=O\left(r^{2 \beta}\right)$. More precisely, the $n$-current $T$ defined by integration over $\mathcal{T}$ (with multiplicity 1 ) has this property. Considering $a \in \mathcal{T}$ and a closed $n$-current $X$ with support in a compact set $K \subset \mathbf{U}(a, r)$ and letting $\pi_{a}$ be the orthonogal projection of $\mathbb{R}^{n+k}$ onto $\operatorname{Tan}_{a} \mathcal{T}$ we observe

$$
\mathbf{M}\left(\pi_{a \#}(T\llcorner K))=\mathbf{M}\left(\pi_{a \#}(T\llcorner K+X)) \leq \mathbf{M}(T\llcorner K+X),\right.\right.
$$

and hence it suffices to prove

$$
\mathbf{M}\left(T\llcorner K) \leq(1+\omega(r)) \mathbf{M}\left(\pi_{a \#}(T\llcorner K))\right.\right.
$$

with $\omega(r)$ as asserted. This is easily done by writing $\mathbf{U}(a, r) \cap \mathcal{T}=\mathbf{U}(a, r) \cap$ (graph $u)$ as a graph over the tangent plane $\operatorname{Tan}_{a} \mathcal{T}$, if $r$ is small enough, and then using the estimate $|D u(y)| \leq$ const $|y|^{\beta}$ in the area integral of the corresponding function $u$. For further discussions of the $(F, \omega)$ minimizing property of solutions to parametric variational problems we refer to [Alm2] and (for the area integrand with codimension 1 and multiplicity one, and with a somewhat different definition of almost minimality) to [Ta2].

We will frequently use the following scaling property: If the rectifiable $n$-current $T$ is $(F, \omega)$ minimizing in $D \subset \mathbb{R}^{n+k}, a \in \mathbb{R}^{n+k}, \vartheta>0, \boldsymbol{\tau}_{a}(x)=x+a$ denotes the translation in $\mathbb{R}^{n+k}, \boldsymbol{\mu}_{\vartheta}(x)=\vartheta x$ denotes the homothety in $\mathbb{R}^{n+k}, \tilde{T}=\boldsymbol{\mu}_{\vartheta \#}^{-1} \boldsymbol{\tau}_{a \#}^{-1} T$, and $\tilde{F}(\tilde{x}, \xi)=$ 
$F(a+\vartheta \tilde{x}, \xi)$ for $\tilde{x} \in \tilde{D}=\boldsymbol{\mu}_{\vartheta}^{-1} \boldsymbol{\tau}_{a}^{-1} D$, then $\tilde{T}$ is $(\tilde{F}, \tilde{\omega})$ minimizing in $\tilde{D}$ with $\tilde{\omega}(\tilde{r})=\omega(\vartheta \tilde{r})$. Here we have taken $\tilde{F}$ instead of the transformed integrand $\tilde{F}=\left(\boldsymbol{\tau}_{a} \circ \boldsymbol{\mu}_{\vartheta}\right)^{\#} F=\vartheta^{n} \tilde{F}$, because then $\tilde{F}$ has the same structure constants $\tilde{\Lambda}=\Lambda, \tilde{\lambda}=\lambda$ and modulus $\tilde{\nu}=\nu$ as $F$ in (1.1)-(1.4). For the moduli appearing in (1.5)-(1.8) we then have $\tilde{\kappa}(r)=\kappa(\vartheta r)$, $\tilde{\mu}(r)=\mu(\vartheta r)$. Furthermore, $\widehat{\tilde{\mu}}(r) \leq \widehat{\mu}(\vartheta r)$ can be easily derived from the definition (1.9) if $0<\vartheta \leq 1$. We also note, for biLipschitz maps $G$ with biLipschitz constant $L$, that $G_{\#}^{-1} T$ is $\left(G^{\#} F, \tilde{\omega}\right)$ minimizing with $\tilde{\omega}(\tilde{r})=L^{n} \omega(L \tilde{r})$.

In the $\epsilon$-regularity theorem (Theorem 6.1) one considers a situation where the $(F, \omega)$ minimizing $n$-current has special projection properties and small cylindrical excess with respect to an $n$-plane in $\mathbb{R}^{n+k}$. We fix this plane to be $\mathbb{R}^{n} \times\{0\}^{k} \subset \mathbb{R}^{n} \times \mathbb{R}^{k}$ and introduce the following notations to be used throughout this paper: $\mathbf{p :} \mathbb{R}^{n+k} \rightarrow \mathbb{R}^{n}$, q: $\mathbb{R}^{n+k} \rightarrow \mathbb{R}^{k}$ are the projections, i.e. $\mathbf{p}(x)=y, \mathbf{q}(x)=z$ for $x=(y, z) \in \mathbb{R}^{n} \times \mathbb{R}^{k}$. Open balls in $\mathbb{R}^{n}$ are denoted by $\mathbf{U}_{\varrho}(y)$, the center $y$ is omitted if $y=0$, the radius $\varrho$ is omitted if $\varrho=1$, so that $\mathbf{U}_{\varrho}$ and $\mathbf{U}$ are open balls in $\mathbb{R}^{n}$ with radius $\varrho$ and 1 respectively and centered at the origin. Closed balls are denoted similarly with $\mathbf{U}$ replaced by B. Cylinders in $\mathbb{R}^{n+k}$ over open balls in $\mathbb{R}^{n}$ will be written $\mathbf{C}_{\varrho}(y)=\mathbf{U}_{\varrho}(y) \times \mathbb{R}^{k}=\mathbf{p}^{-1}\left(\mathbf{U}_{\varrho}(y)\right)$ with the same conventions for the suppressing of $y$ or $\varrho$, in particular we will usually work in the unit cylinder $\mathbf{C}=\mathbf{U} \times \mathbb{R}^{k}$. Closed cylinders are denoted using $\overline{\mathbf{C}}$ instead of $\mathbf{C}$. The superscript or the subscript "+" at open balls in $\mathbb{R}^{n}$ indicates intersection with the half space $\left.\mathbb{R}^{n-1} \times\right] 0, \infty\left[\right.$, so that e.g. $\mathbf{U}_{+}$ is the open unit half ball in $\mathbb{R}^{n}$. Similarly, $\left.\mathbf{C}_{\varrho}^{+}=\mathbf{C}_{\varrho}(y) \cap \mathbb{R}^{n-1} \times\right] 0, \infty\left[\times \mathbb{R}^{k}\right.$. The closure of $\mathbf{U}_{\varrho}^{+}(y)$ and $\mathbf{C}_{\varrho}^{+}(y)$ is written $\mathbf{B}_{\varrho}^{+}(y)$ and $\overline{\mathbf{C}}_{\varrho}^{+}(y)$ respectively, in particular $\mathbf{B}_{+}$is the closed unit half ball in $\mathbb{R}^{n}$ and $\overline{\mathbf{C}}_{+}=\mathbf{B}_{+} \times \mathbb{R}^{k}$. Superscripts or subscripts "-" have analogous meaning with $]-\infty, 0[$ replacing $] 0, \infty\left[\right.$. Balls in a Euclidean space $\mathbb{R}^{l}$ of arbitrary dimension we denote $\mathbf{U}_{r}^{l}(a), \mathbf{B}_{r}^{l}(y)$ or $\mathbf{U}^{l}(a, r), \mathbf{B}^{l}(a, r)$, indicating the dimension. However, in the case $l=n+k$ we omit the dimension writing $\mathbf{U}(x, r)$, $\mathbf{B}(x, r)$; no confusion should arise from this, as it can seen from the context that $x \in \mathbb{R}^{n+k}$ in this case.

For the interior $\epsilon$-regularity theorem we consider the class of admissible currents in the interior situation

$$
\mathcal{T}_{i}(n, k, m)
$$

which consists of the $n$-dimensional currents $T$ on $\mathbb{R}^{n+k}$ with the following properties:

$$
\begin{aligned}
& T \in \mathcal{R}_{n}\left(\mathbb{R}^{n+k}\right), \quad T=T\llcorner\mathbf{C}, \\
& \partial T\llcorner\mathbf{C}=0, \quad 0 \in \operatorname{spt} T, \\
& \mathbf{p}_{\#} T=m\left(\mathbf{E}^{n}\llcorner\mathbf{U}) .\right.
\end{aligned}
$$

The first condition means that $T$ is rectifiable and vanishes outside $\mathbf{C}$, by the second condition $T$ has zero boundary in $\mathbf{C}$ and does not vanish on a neighborhood of the origin, the third condition tells us that the projection of $T$ onto $\mathbb{R}^{n}$ is represented by the unit disc with standard orientation and multiplicity $m$. Here $m$ is a given positive integer, and $\mathbf{E}^{n}$ denotes the $n$-current on $\mathbb{R}^{n}$ defined by integration of $n$-forms (with respect to the standard orientation of $\mathbb{R}^{n}$ ).

For the boundary regularity theorem we consider an analogous class of currents $T$ with $\partial T\llcorner\mathbf{C}$ prescribed (and nonzero). Thinking of a boundary condition $\partial T=B$ where $B$ 
is represented by an oriented submanifold $\mathcal{B}$ of class $C^{1,1}$ in $\mathbb{R}^{n+k}$ it is no restriction, by the invariance of the structure conditions for the integrand $F$ and the $(F, \omega)$ minimizing property of $T$ with respect to transformations by biLipschitz diffeomorphisms of class $C^{1,1}$, to assume that $\mathcal{B}$ is an $(n-1)$-dimensional affine subspace which we take as $\mathbb{R}^{n-1} \times\{0\}^{k+1} \subset$ $\mathbb{R}^{n+k}$ with the boundary orientation induced from the half space $\left.\mathbb{R}^{n-1} \times\right] 0, \infty\left[\right.$ (this is $(-1)^{n}$ times the standard orientation of $\mathbb{R}^{n-1}$ ). We therefore replace (1.19) by

$$
\partial T\left\llcorner\mathbf{C}=B \quad \text { with } \quad B=(-1)^{n-1}\left(\mathbf{E}^{n-1} \times \boldsymbol{\delta}_{0}^{k+1}\right)\llcorner\mathbf{C}\right.
$$

and (1.20) by

$$
\mathbf{p}_{\#} T=m\left(\mathbf{E}^{n}\left\llcorner\mathbf{U}_{+}\right)+(m-1)\left(\mathbf{E}^{n}\left\llcorner\mathbf{U}_{-}\right),\right.\right.
$$

that is $\mathbf{p}_{\#} T$ is represented by the two half disks $\mathbf{U}_{+}, \mathbf{U}_{-}$with standard orientation and multiplicities $m, m-1$ respectively, and

$$
\mathbf{p}_{\#} B=\partial\left(\mathbf{E}^{n}\left\llcorner\mathbf{U}_{+}\right)\llcorner\mathbf{U}\right.
$$

is the oriented boundary of the half disc $\mathbf{U}_{+}$in $\mathbf{U}$. The class of admissible currents in the boundary situation

$$
\mathcal{T}_{b}(n, k, m)
$$

is then defined as the set of all currents $T$ satisfying (1.18), (1.21) and (1.22). We use the notation

$$
\mathcal{T}_{*}(n, k, m)=\mathcal{T}_{i}(n, k, m) \cup \mathcal{T}_{b}(n, k, m)
$$

so that statements made about $T \in \mathcal{T}_{*}(n, k, m)$ are valid in the interior situation and in the boundary situation as well. We notice that $\left(\boldsymbol{\mu}_{\varrho \#}^{-1} \boldsymbol{\tau}_{a \#}^{-1} T\right)\left\llcorner\mathbf{C} \in \mathcal{T}_{i}(n, k, m)\right.$ if $T \in \mathcal{T}_{i}(n, k, m)$, $a \in \mathbf{C} \cap \operatorname{spt} T$, and $0<\varrho \leq 1-|\mathbf{p} a|$. The analogous assertion holds for $\mathcal{T}_{b}$ instead of $\mathcal{T}_{i}$, provided $a \in \mathbf{C} \cap \mathbb{R}^{n-1} \times\{0\}^{k+1}$.

A crucial role in the regularity theory for rectifiable $n$-currents $T$ on $\mathbb{R}^{n+k}$ is played by the cylindrical excess which is defined, for $r>0$, by

$$
\mathbf{E}(T, r)=r^{-n}\left[\mathbf { M } \left(T\left\llcorner\mathbf{C}_{r}\right)-\mathbf{M}\left(\mathbf{p}_{\#}\left(T\left\llcorner\mathbf{C}_{r}\right)\right)\right] \geq 0,\right.\right.
$$

and, for $y \in \mathbb{R}^{n}$,

$$
\mathbf{E}(T, y, r)=\mathbf{E}\left(\boldsymbol{\tau}_{(y, 0) \#}^{-1} T, r\right) .
$$

If $\mathbf{p}_{\#}\left(T\left\llcorner\mathbf{C}_{r}\right)=\mathbf{E}^{n}\llcorner\vartheta\right.$ holds with a nonnegative multiplicity function $\vartheta$, then one can write

$$
\mathbf{E}(T, r)=r^{-n} \int_{\mathbf{C}_{r}}(1-\vec{T} \cdot \overrightarrow{\mathbf{e}}) d\|T\|=\frac{1}{2} r^{-n} \int_{\mathbf{C}_{r}}|\vec{T}-\overrightarrow{\mathbf{e}}|^{2} d\|T\|,
$$

where $\overrightarrow{\mathbf{e}}$ is the orientation vector of $\mathbf{E}^{n} \times \delta_{0}^{k}$ (i.e. the exterior product $\mathbf{e}_{1} \wedge \ldots \wedge \mathbf{e}_{n}$ of the first $n$ canonical basis vectors of $\left.\mathbb{R}^{n+k}\right)$. In particular, we may use $(1.24)$ for $T \in \mathcal{T}_{*}(n, k, m)$, and we have

$$
\mathbf{E}(T, r)=r^{-n} \mathbf{M}\left(T\left\llcorner\mathbf{C}_{r}\right)-m \boldsymbol{\alpha}(n) \quad \text { if } T \in \mathcal{T}_{i}(n, k, m),\right.
$$




$$
\mathbf{E}(T, r)=r^{-n} \mathbf{M}\left(T\left\llcorner\mathbf{C}_{r}\right)-\frac{1}{2}(2 m-1) \boldsymbol{\alpha}(n) \quad \text { if } T \in \mathcal{T}_{b}(n, k, m),\right.
$$

where $\boldsymbol{\alpha}(n)=\mathcal{L}^{n}(\mathbf{U})$ is the volume of the unit ball in $\mathbb{R}^{n}$. We note the mass bound $\mathbf{M}\left(T\left\llcorner\mathbf{C}_{r}\right) \leq 1+m \boldsymbol{\alpha}(n) r^{n}\right.$ as a consequence, if $\mathbf{E}(T, r) \leq 1$. We also note the monotonicity property $r^{n} \mathbf{E}(T, r) \leq s^{n} \mathbf{E}(T, s)$ for $0<r \leq s$. (The main part in the regularity theory is to prove that also the scaled quantity $\mathbf{E}(T, r)$ is decreasing to zero with $r \downarrow 0$.)

Finally, with $C_{1}, C_{2}, \ldots$ we denote various constants which are "large", i.e. $C_{i} \in[1, \infty[$, while $\delta, \epsilon, \ldots$ are small positive constants with values in $] 0,1\left[\right.$. We usually write $C_{i}=$ const $(n, k, \Lambda, \nu, \ldots)$ or $\delta=\delta(n, k, \Lambda, \nu, \ldots)$ when the constants appear for the first time, in order to indicate their dependence on parameters or moduli $n, k, \Lambda, \nu, \ldots$.

\section{Lower mass estimate and height bound}

In this section we establish a lower bound for the mass in balls and an upper bound for the height of $(F, \omega)$ minimizing currents $T \in \mathcal{T}_{*}(n, k, m)$. The arguments are well known in the interior case (cf. [Fe, 5.1.6], [Fe, 5.3.4], [Bo, Sec.IV], [SS, Appendix]) and easily adapted to the boundary situation, we give proofs here only for the sake of completeness. For the mass estimate (Lemma 2.1) one only needs the structure condition (1.1) on $F$, i.e. $|\xi| \leq F(\cdot, \xi) \leq \Lambda|\xi|$, and the boundedness of $\omega$. The height estimate (Lemma 2.2) then follows assuming smallness of the excess of $T$ and, in the case of multiplicity $m>1$, additional conditions on the density of $\|T\|$ (Remark 2.3).

2.1 Lemma (lower mass estimate). Suppose $T \in \mathcal{T}_{*}(n, k, m)$ is $(F, \omega)$ minimizing in C. Then

$$
C_{1} \mathbf{M}\left(T\llcorner\mathbf{U}(x, r)) \geq[\Lambda+\omega(r)]^{1-n} r^{n}\right.
$$

holds for all $x \in \operatorname{spt} T$ and $0<r<1-|\mathbf{p} x|$, where $C_{1}=\operatorname{const}(n)$.

Proof. We use the Euclidean distance function $d_{x}$ to $x$ to obtain, for almost every $r$ as above, a rectifiable slice $\left\langle T, d_{x}, r\right\rangle=\partial(T\llcorner\mathbf{U}(x, r))-(\partial T)\llcorner\mathbf{U}(x, r)$ with $T\llcorner\mathbf{U}(x, r)=$ $T\left\llcorner\mathrm{~B}(x, r)\right.$ (see [Fe, Sec. 4.3]). We then consider a rectifiable current $S$ of least mass in $\mathbb{R}^{n+k}$ with boundary $\partial S=\partial(T\llcorner\mathbf{U}(x, r))$. From the isoperimetric inequality [Fe, 4.4.2], [Alm4] we have $\mathbf{M}(S) \leq \gamma_{n} \mathbf{M}(\partial S)^{n /(n-1)}$, and the convexity of $\mathbf{U}(x, r)$ implies spt $S \subset \mathbf{B}(x, r)$. We may therefore use the $(F, \omega)$ minimality of $T$ and the structure conditions on $F$ to infer

$$
\begin{aligned}
\mathbf{M}(T\llcorner\mathbf{U}(x, r)) & \leq \mathbf{F}(T\llcorner\mathbf{U}(x, r)) \leq \mathbf{F}(S)+\omega(r) \mathbf{M}(S) \\
& \leq[\Lambda+\omega(r)] \mathbf{M}(S) \leq[\Lambda+\omega(r)] \boldsymbol{\gamma}_{n} \mathbf{M}(\partial S)^{n /(n-1)} \\
& \leq[\Lambda+\omega(r)] \boldsymbol{\gamma}_{n}\left[\mathbf{M}\left(\left\langle T, d_{x}, r\right\rangle\right)+\mathbf{M}((\partial T)\llcorner\mathbf{U}(x, r))]^{n /(n-1)}\right.
\end{aligned}
$$

In the case $(\partial T)\left\llcorner\mathbf{U}(x, r) \neq 0\right.$ we use additionally the projection from $\mathbb{R}^{n+k}$ onto the subspace $\mathbb{R}^{n-1} \times\{0\}^{k+1}$ to see $\mathbf{M}\left((\partial T)\llcorner\mathbf{U}(x, r)) \leq \mathbf{M}\left(\left\langle T, d_{x}, r\right\rangle\right)\right.$, because this projection maps $\left\langle T, d_{x}, r\right\rangle$ onto $-(\partial T)\llcorner\mathbf{U}(x, r)$. In any case

$$
\mathbf{M}\left(T\llcorner\mathbf{U}(x, r)) \leq[\Lambda+\omega(r)] \boldsymbol{\gamma}_{n} 2^{n /(n-1)} \mathbf{M}\left(\left\langle T, d_{x}, r\right\rangle\right)^{n /(n-1)}\right.
$$


is valid.

Introducing $f(r)=\mathbf{M}\left(T\llcorner\mathbf{U}(x, r))\right.$ and recalling $\mathbf{M}\left(\left\langle T, d_{x}, r\right\rangle\right) \leq f^{\prime}(r)$ for almost all $r$ $([\mathrm{Fe}, 4.2 .1])$ we deduce from $(2.2)$

$$
f(r) \leq[\Lambda+\omega(r)] \boldsymbol{\gamma}_{n} 2^{n /(n-1)} f^{\prime}(r)^{n /(n-1)} .
$$

Noting $f(r)>0$ for $r>0$, since $x \in \operatorname{spt} T$, we may rewrite this differential inequality as

$$
\frac{d}{d r}\left[f(r)^{1 / n}\right] \geq[\Lambda+\omega(r)]^{(1-n) / n} \gamma_{n}^{(1-n) / n} 2 n^{-1}
$$

valid for almost all $r$. Finally, we integrate (2.3) using the monotonicity of $f$ and $f(0)=0$ to obtain (2.1).

2.2 Lemma (height bound). Suppose $T \in \mathcal{T}_{*}(n, k, 1)$ is $(F, \omega)$ minimizing in $\mathbf{C}$ with a smallness condition for the excess

$$
C_{2}\left[\Lambda+\omega\left(\frac{1}{2}\right)\right]^{n-1} \mathbf{E}(T, 1)^{1 / 2} \leq 1
$$

where $C_{2}=\operatorname{const}(n)$. Then the estimate

$$
\sup \left\{|\mathbf{q} x|^{n}: x \in \mathbf{C}_{1 / 2} \cap \operatorname{spt} T\right\} \leq C_{3}\left[\Lambda+\omega\left(\frac{1}{2}\right)\right]^{n-1} \mathbf{E}(T, 1)^{1 / 2}
$$

is valid with $C_{3}=\operatorname{const}(n)$.

Proof. We first treat the boundary situation $T \in \mathcal{T}_{b}(n, k, 1)$. Here we have $\mathbf{M}(T)=$ $\mathbf{E}(T, 1)+\frac{1}{2} \boldsymbol{\alpha}(n)$ and, consequently, $\mathbf{M}(T) \leq \frac{3}{4} \boldsymbol{\alpha}(n)$ if $C_{2}$ in (2.4) is chosen to imply $\mathbf{E}(T, 1) \leq \frac{1}{4} \boldsymbol{\alpha}(n)$. Fix a unit vector $u \in\{0\}^{n} \times \mathbb{R}^{k} \subset \mathbb{R}^{n+k}$ and define $H(r)=\{x \in$ $\mathbf{C}: u \cdot x>r\}, T_{r}=T\llcorner H(r)$, and $H(r, s)=\{x \in \mathbf{C}: r<u \cdot x<s\}$ for $r<s$. Since $\mathbf{M}\left(\mathbf{p}_{\#} T_{r}\right) \leq \mathbf{M}(T)$ and thus

$$
\mathbf{M}\left(\mathbf{p}_{\#} T_{r}\right) \leq \frac{3}{4} \boldsymbol{\alpha}(n),
$$

we may apply the relative isoperimetric inequality [Fe, 5.3.2] to obtain

$$
\mathbf{M}\left(\mathbf{p}_{\#} T_{r}\right)^{1-1 / n} \leq \operatorname{const}(n) \mathbf{M}\left(\partial\left(\mathbf{p}_{\#} T_{r}\right)\llcorner\mathbf{U}) \leq \operatorname{const}(n) \mathbf{M}\left(\left(\partial T_{r}\right)\llcorner\mathbf{C}) .\right.\right.
$$

Suppose now $\mathbf{M}\left(T_{s}\right) \geq \sqrt{E}$ for some $s>0$, where $E=\mathbf{E}(T, 1) \leq \frac{1}{4}$ and we may assume $E>0$. Then, for $0<r<s$ we get from $(2.7)$

$$
\begin{aligned}
(\sqrt{E}-E)^{1-1 / n} & \leq\left[\mathbf{M}\left(T_{r}\right)-\mathbf{E}(T, 1)\right]^{1-1 / n} \leq \mathbf{M}\left(\mathbf{p}_{\#} T_{r}\right)^{1-1 / n} \\
& \leq \operatorname{const}(n) \mathbf{M}\left(\left(\partial T_{r}\right)\llcorner\mathbf{C})\right.
\end{aligned}
$$

Integrating this inequality for $r \in] 0, s$ [ and using the identity

$$
\left\langle T, \ell_{u}, r\right\rangle\left\llcorner\mathbf{C}=\partial T\left\llcorner H(r)-\left(\partial T_{r}\right)\left\llcorner\mathbf{C}=-\left(\partial T_{r}\right)\llcorner\mathbf{C}\right.\right.\right.
$$


for the slices of $T$ with respect to the linear function $\ell_{u}(x)=u \cdot x$ (note that $H(r) \cap$ spt $\partial T=\emptyset$ for $r>0$ ) we obtain from the standard slicing estimate [Fe, 4.3.2(2)] and $\mathbf{M}(T) \leq \operatorname{const}(n)$

$$
\begin{aligned}
\left(\frac{1}{2} \sqrt{E}\right)^{1-1 / n} s & \leq(\sqrt{E}-E)^{1-1 / n} s \leq \operatorname{const}(n) \int_{0}^{s} \mathbf{M}\left(\left\langle T, \ell_{u} r\right\rangle\right) d r \\
& \leq \operatorname{const}(n) \int_{H(0, s)} \mid \vec{T}\left\llcorner d \ell_{u} \mid d\|T\|\right. \\
& \leq \operatorname{const}(n)\left[\int_{H(0, s)} \mid \vec{T}\left\llcorner\left. d \ell_{u}\right|^{2} d\|T\|\right]^{1 / 2}\right. \\
& \leq \operatorname{const}(n)\left[\int_{H(0, s)}\left(1-(\vec{T} \cdot \overrightarrow{\mathbf{e}})^{2}\right) d\|T\|\right]^{1 / 2} \\
& \leq \operatorname{const}(n)\left[2 \int_{H(0, s)}(1-\vec{T} \cdot \overrightarrow{\mathbf{e}}) d\|T\|\right]^{1 / 2} \\
& \leq \operatorname{const}(n) \mathbf{E}\left(T\llcorner H(0, s), 1)^{1 / 2} \leq \operatorname{const}(n) \sqrt{E} .\right.
\end{aligned}
$$

This implies

$$
s \leq \operatorname{const}(n) E^{1 / 2 n} \quad \text { when } s>0 \text { and } \mathbf{M}\left(T_{s}\right) \geq \sqrt{E} .
$$

On the other hand, if $s>0$ with $\mathbf{M}\left(T_{s}\right) \leq \sqrt{E}$ and $x \in \overline{\mathbf{C}}_{1 / 2} \cap \operatorname{spt} T$ with $u \cdot x \geq s+\delta$ for some $0<\delta<\frac{1}{2}$, then $\mathbf{U}(x, \delta) \subset H(s)$, and we see from Lemma 2.1

$$
[\Lambda+\omega(\delta)]^{1-n} \delta^{n} \leq C_{1} \mathbf{M}\left(T\llcorner\mathbf{U}(x, \delta)) \leq C_{1} \mathbf{M}\left(T_{s}\right) \leq C_{1} \sqrt{E},\right.
$$

an impossibility if we choose $\delta^{n}=2\left[\Lambda+\omega\left(\frac{1}{2}\right)\right]^{n-1} C_{1} \sqrt{E}$ and we require (2.4) with $C_{2}$ large enough to secure $\delta<\frac{1}{2}$. We deduce

$$
\begin{gathered}
\sup \left\{u \cdot x: x \in \overline{\mathbf{C}}_{1 / 2} \cap \operatorname{spt} T\right\} \leq s+\operatorname{const}(n)\left[\Lambda+\omega\left(\frac{1}{2}\right)\right]^{1-1 / n} E^{1 / 2 n} \\
\text { when } s>0 \text { and } \mathbf{M}\left(T_{s}\right) \leq \sqrt{E}
\end{gathered}
$$

Clearly, with (2.9) and (2.10) we have proved

$$
\sup \left\{u \cdot x: x \in \overline{\mathbf{C}}_{1 / 2} \cap \operatorname{spt} T\right\} \leq \operatorname{const}(n)\left[\Lambda+\omega\left(\frac{1}{2}\right)\right]^{1-1 / n} E^{1 / 2 n},
$$

and (2.5) follows since $u \in\{0\}^{n} \times \mathbb{R}^{k}$ is arbitrary with $|u|=1$.

In the interior situation $T \in \mathcal{T}_{i}(n, k, 1)$ one first chooses a median value $r_{0}$ such that both $\mathbf{M}\left(T\left\llcorner H\left(r_{0}, \infty\right)\right)\right.$ and $\mathbf{M}\left(T\left\llcorner H\left(-\infty, r_{0}\right)\right)\right.$ do not exceed $\frac{1}{2} \mathbf{M}(T)$. Then $\mathbf{M}\left(\mathbf{p}_{\#} T_{r_{0}}\right) \leq$ $\frac{1}{2} \boldsymbol{\alpha}(n)+\frac{1}{2} E$ holds, and (2.6) is valid for $r>r_{0}$ assuming again $E \leq \frac{1}{4} \boldsymbol{\alpha}(n)$. Reasoning as in (2.9), (2.10) one obtains an estimate analogous to (2.11) for the oscillation of $\ell_{u}$ on $\overline{\mathbf{C}}_{1 / 2} \cap \operatorname{spt} T$. The inequality (2.5) then follows from $0 \in \operatorname{spt} T$. (Cf. the proof of Lemma 2 in $[\mathrm{SS}]$. 
2.3 Remark. In the case case of multiplicity $\boldsymbol{m}>\mathbf{1}$ one can prove a height estimate similar to (2.5), provided the additional assumption

$$
\Theta^{n}(\|T\|, x) \geq m \text { for }\|T\| \text { almost all } x \in \mathbf{C}
$$

is satisfied when $T \in \mathcal{T}_{i}(n, k, m)$, and

$$
\begin{array}{ll}
\Theta^{n}(\|T\|, x) \geq m & \text { for }\|T\| \text { almost all } x \in \mathbf{C}_{+}, \\
\Theta^{n}(\|T\|, x) \geq m-1 & \text { for }\|T\| \text { almost all } x \in \mathbf{C}_{-}
\end{array}
$$

when $T \in \mathcal{T}_{b}(n, k, m)$.

For $T \in \mathcal{T}_{i}(n, k, m)$ and the median value $r_{0}$ from the proof above we deduce from (2.12) and $E \leq \frac{m}{4} \boldsymbol{\alpha}(n)$ that $\mathcal{L}^{n}(P(r)) \leq \frac{3}{4} \boldsymbol{\alpha}(n)$ for $r \geq r_{0}$ and the set $P(r) \subset \mathbf{U}$ of points $y$ with $\Theta^{n}\left(\| \mathbf{p}_{\#}(T\llcorner H(r, \infty)) \|, y) \geq 1\right.$. Therefore, the relative isoperimetric inequality (2.7) is again valid, and we may argue as before to obtain (2.5) with $C_{3}=m^{n / 2} \operatorname{const}(n)$.

For $T \in \mathcal{T}_{b}(n, k, m)$ we first choose median values $r_{0}^{+}, r_{0}^{-}$for the half cylinders $\mathbf{C}_{+}, \mathbf{C}_{-}$ analogous to $r_{0}$ for $\mathbf{C}$. We then argue as in the proof of $(2.9)$ to obtain, with $c=\operatorname{const}(n)$ and $H_{ \pm}(\ldots)=\mathbf{C}_{ \pm} \cap H(\ldots)$,

$$
\begin{array}{ll}
\mathbf{M}\left(T\left\llcorner H_{+}(r, \infty)\right) \leq \frac{1}{2} \sqrt{E}\right. & \text { for } r \geq r_{0}^{+}+c E^{1 / 2 n}, \\
\mathbf{M}\left(T\left\llcorner H_{+}(-\infty, r)\right) \leq \frac{1}{2} \sqrt{E}\right. & \text { for } r \leq r_{0}^{+}-c E^{1 / 2 n},
\end{array}
$$

and analogous statements for $T\left\llcorner H_{-}(\ldots)\right.$ and $r_{0}^{-}$.

We claim that $\left|r_{0}^{+}-r_{0}^{-}\right| \leq 2 c E^{1 / 2 n}+\delta$ where $\delta=\left(2 C_{1}\right)^{1 / n}\left[\Lambda+\omega\left(\frac{1}{2}\right)\right]^{1-1 / n} E^{1 / 2 n}<\frac{1}{2}$ is as in the proof of Lemma 2.2 and $E=\mathbf{E}(T, 1)$ is sufficiently small (and positive). With this estimate for $\left|r_{0}^{+}-r_{0}^{-}\right|$we can then derive the height estimate (2.5) with $C_{3}=m^{n / 2} \operatorname{const}(n)$ exactly as in the proof of (2.10).

For $x \in \mathbf{C}_{1-\delta} \cap \operatorname{spt} T$ we first note $\mathbf{M}(T\llcorner\mathbf{U}(x, \delta)) \geq 2 \sqrt{E}$, by Lemma 2.1 and the definition of $\delta$, and we infer

$$
\mathbf{M}\left(T \llcorner ( \mathbf { C } _ { + } \cap \mathbf { U } ( x , \delta ) ) ) > \frac { 1 } { 2 } \sqrt { E } \quad \text { or } \quad \mathbf { M } \left(T\left\llcorner\left(\mathbf{C}_{-} \cap \mathbf{U}(x, \delta)\right)\right)>\frac{1}{2} \sqrt{E},\right.\right.
$$

since $\mathbf{M}\left(T\left\llcorner\left(\mathbf{C} \backslash\left(\mathbf{C}_{+} \cup \mathbf{C}_{-}\right)\right)\right) \leq E<\sqrt{E}\right.$. Assuming $\left|r_{0}^{+}-r_{0}^{-}\right|>2 c E^{1 / 2 n}+2 \delta$ we deduce $u \cdot x \neq s$ for $s=\frac{1}{2}\left(r_{0}^{+}+r_{0}^{-}\right)$, because otherwise $\mathbf{U}(x, \delta)$ would be disjoint from $H_{+}\left(r_{0}^{+}-c E^{1 / 2 n}, r_{0}^{+}+c E^{1 / 2 n}\right)$ and from $H_{-}\left(r_{0}^{-}-c E^{1 / 2 n}, r_{0}^{-}+c E^{1 / 2 n}\right)$ and then (2.15) would contradict (2.14). Since $0 \in \operatorname{spt} \partial T$ and $u \cdot x=0$ for $x \in \mathbf{C} \cap \operatorname{spt} \partial T$ we conclude $s>0$ and $\partial\left(T\llcorner H(s, \infty))\left\llcorner\mathbf{C}_{1-\delta}=0\right.\right.$, or $s<0$ and $\partial\left(T\llcorner H(-\infty, s))\left\llcorner\mathbf{C}_{1-\delta}=0\right.\right.$. The second case being similar, we proceed with the first case where $\left[\partial\left(\mathbf{p}_{\#} T_{s}\right)\right]\left\llcorner\mathbf{U}_{1-\delta}=0\right.$ and $\mathbf{p}_{\#} T_{s}$ is constant on $\mathbf{U}_{1-\delta}$. But it follows from $(2.14),\left|r_{0}^{+}-r_{0}^{-}\right|>2 c E^{1 / 2 n}$, and the choice of $s$ that

$$
\mathbf{M}\left(( \mathbf { p } _ { \# } T _ { s } ) \llcorner \mathbf { U } _ { + } ) \leq \frac { 1 } { 2 } \sqrt { E } \quad \text { and } \quad \mathbf { M } \left(T\left\llcorner H_{-}(-\infty, s)\right) \leq \frac{1}{2} \sqrt{E},\right.\right.
$$

or

$$
\mathbf{M}\left(( \mathbf { p } _ { \# } T _ { s } ) \llcorner \mathbf { U } _ { - } ) \leq \frac { 1 } { 2 } \sqrt { E } \quad \text { and } \quad \mathbf { M } \left(T\left\llcorner H_{+}(-\infty, s)\right) \leq \frac{1}{2} \sqrt{E}\right.\right.
$$


For $\sqrt{E}<2^{-n-1} \boldsymbol{\alpha}(n)$ this contradicts the constancy of $\mathbf{p}_{\#} T_{s}$ on $\mathbf{U}_{1 / 2}$, because $\mathbf{p}_{\#} T$ has

multiplicity $\geq m-1>0$ everywhere on $\mathbf{U}$ and $\left\{x \in \mathbf{C}_{1 / 2} \cap \operatorname{spt} T: u \cdot x=s\right\}$ is empty. The claim is proved.

We note that the bad hypotheses (2.12), (2.13) are needed only for the relative isoperimetric inequality (2.7) and somewhat weaker assumptions would suffice here. For example, it would be enough to know the inequalities $(2.12)$, (2.13) on subcylinders of $\mathbf{C}, \mathbf{C}_{+}, \mathbf{C}_{-}$ with cross sections of positive $\mathcal{L}^{n}$ measure (independent of $T$ ). However, we shall need scaled versions of the height estimate which we can prove, in the case $m \geq 2$, only with extra assumptions similar to (2.12), (2.13) (cf. [Fe, 5.3.17] and the discussion following Lemma 2 in $[\mathrm{SS}])$.

\section{Harmonic approximation}

As a general hypothesis we assume in this section that $T \in \mathcal{T}_{*}(n, k, m)$ is $(F, \omega)$ minimizing in $\mathbf{C}$, where the integrand $F$ satisfies $|\xi| \leq F(\cdot, \xi) \leq \Lambda|\xi|$ and $\omega:] 0,1[\rightarrow[0, \infty[$ is nondecreasing, and that the additional density conditions (2.12), (2.13) hold in the case of multiplicity $m \geq 2$. Using the height estimate Lemma 2.2 we first show that $T$ can be approximated, with error estimates in terms of the excess $\mathbf{E}(T, 1)$, by the current $T_{g}$ associated with the graph of a Lipschitz function $g: \mathbb{R}^{n} \rightarrow \mathbb{R}^{k}$ equipped with appropriately chosen multiplicities and orientation. In the interior case this was done in [SS, Lemma 3] modifying [All1, 8.12] (cf. [Si1, §20]), and it is not difficult to adapt the arguments to the boundary situation treated here. Since the estimates we need are somewhat different from those in [SS] we give complete proofs. We next show that the approximating function $g$ is, again with an error dominated by the excess, an approximate solution to the homogeneous constant coefficient elliptic system determined by the nonparametric elliptic integrand associated with $F$ frozen at the origin (i.e. with $\xi \mapsto F(0, \xi)$ ). This is similar to Lemma 12 and Lemma 13 in [Bo], in the boundary situation $T \in \mathcal{T}_{b}(n, k, m)$, however, we can prove such a statement only on the half ball $\mathbf{U}_{+}$(and also on $\mathbf{U}_{-}$if $m \geq 2$ ). Finally, we state a simple Lemma following [Si1, §21], [Si2, §1.6] which asserts that the approximate solution $g$ is indeed close, in the weak topology of the Sobolev space $W^{1,2}$, to an exact solution.

3.1 Lemma (Lipschitz approximation). Given $0<\varrho \leq \frac{1}{17}$ and $0<\beta \leq 1$ there exists a Lipschitz map $g: \mathbf{U}_{\varrho} \rightarrow \mathbb{R}^{k}$ with $\operatorname{Lip} g \leq \beta$ such that the following assertions are true:

(i) The $n$-current $T_{g}$ represented by graph $g$, with orientation and multiplicities induced from $\left(\mathbf{p}_{\#} T\right)\left\llcorner\mathbf{U}_{\varrho}\right.$ by the projection $\mathbf{p}$, satisfies

$$
\left\|T-T_{g}\right\|\left(\mathbf{C}_{\varrho}\right) \leq C_{4} \beta^{-2 n} \mathbf{E}(T, 1)
$$

with a constant $C_{4}=\operatorname{const}(n, m)\left[\Lambda+\omega\left(\frac{1}{2}\right)\right]^{2 n-2}$.

(ii) Let $A_{g}$ be the set of $y \in \mathbf{U}_{\varrho}$ satisfing $\{y\} \times \mathbb{R}^{k} \cap \operatorname{spt} T \backslash$ graph $g \neq \emptyset$ and in the case $T \in \mathcal{T}_{b}(n, k, m)$ additionally $y \in \mathbf{U}_{+}$; then

$$
\mathcal{L}^{n}\left(A_{g}\right) \leq C_{5} \beta^{-2 n} \mathbf{E}(T, 1)
$$

holds with a constant $C_{5}=\operatorname{const}(n, m)\left[\Lambda+\omega\left(\frac{1}{2}\right)\right]^{2 n-2}$. 
(iii) In the case $T \notin \mathcal{T}_{i}(n, k, m)$

$$
\int_{\mathbf{U}_{\varrho} \backslash A_{g}}|D g|^{2} d \mathcal{L}^{n} \leq 3 \mathbf{E}(T, 1)
$$

holds; in the case $T \in \mathcal{T}_{b}(n, k, m)$ this is valid with $\mathbf{U}_{+} \cap \mathbf{U}_{\varrho} \backslash A_{g}$ as domain of integration.

(iv) For $\gamma>0$ let $A_{g, \gamma}=A_{g} \cup\left\{y \in \mathbf{U}_{\varrho}:|D g(y)| \geq \gamma\right\}$ in the case $T \in \mathcal{T}_{i}(n, k, m)$ and $A_{g, \gamma}=A_{g} \cup\left\{y \in \mathbf{U}_{\varrho} \cap \mathbf{U}_{+}:|D g(y)| \geq \gamma\right\}$ in the case $T \in \mathcal{T}_{b}(n, k, m)$; then

$$
\begin{aligned}
& \mathcal{L}^{n}\left(A_{g, \gamma}\right) \leq\left(1+C_{5} \beta^{-2 n}\right) 3 \gamma^{-2} \mathbf{E}(T, 1) \\
& \|T\|\left(A_{g, \gamma} \times \mathbb{R}^{k}\right) \leq\left[1+m\left(1+C_{5} \beta^{-2 n}\right) 3 \gamma^{-2}\right] \mathbf{E}(T, 1) .
\end{aligned}
$$

(v) $g(0)=0$ and in the boundary case $T \in \mathcal{T}_{b}(n, k, m)$ additionally $g(y)=0$ for all $y \in \mathbf{U}_{\varrho}^{n-1} \times\{0\}$.

(vi) If $C_{2}\left[\Lambda+\omega\left(\frac{1}{2}\right)\right]^{n-1} \mathbf{E}(T, 1)^{1 / 2} \leq 1$ holds, then

$$
\sup _{\mathbf{U}_{\varrho}}|g|^{n} \leq C_{3}\left[\Lambda+\omega\left(\frac{1}{2}\right)\right]^{n-1} \mathbf{E}(T, 1)^{1 / 2}
$$

where $C_{2}, C_{3}$ are the constants from Lemma 2.2.

Proof. We only treat the boundary case $T \in \mathcal{T}_{b}(n, k, m)$ as the proof in the interior situation is similar, but simpler.

For $0<\varrho \leq \frac{1}{4}$ and $\eta$ to be fixed later define the good set

$$
G=\left\{y \in \mathbf{U}_{\varrho}: y_{n} \neq 0 \text { and } \mathbf{E}(T, y, \sigma) \leq \eta \text { for } 0<\sigma<1-\varrho\right\} .
$$

Then for each $y \in \mathbf{U}_{\varrho} \backslash G$ there exists $\left.\sigma(y) \in\right] 0,1-\varrho[$ with

$$
\frac{1}{2} \int_{\mathbf{C}_{\sigma(y)}(y)}|\vec{T}-\overrightarrow{\mathbf{e}}|^{2} d\|T\|=\sigma(y)^{n} \mathbf{E}(T, y, \sigma(y))>\sigma(y)^{n} \eta
$$

By the Besicovitch covering theorem we can find $N=\operatorname{const}(n)$ disjointed systems $\mathcal{B}_{1}, \ldots$, $\mathcal{B}_{N}$ of balls $\mathbf{B}(y, \sigma(y)), y \in \mathbf{U}_{\varrho} \backslash G$, such that $\mathcal{B}_{1} \cup \ldots \cup \mathcal{B}_{N}$ covers $\mathbf{U}_{\varrho} \backslash G$. For $i \in\{1, \ldots, N\}$ chosen suitably we infer the following estimate for the bad set:

$$
\begin{aligned}
\mathcal{L}^{n}\left(\mathbf{U}_{\varrho} \backslash G\right) & \leq N \mathcal{L}^{n}\left(\bigcup_{B \in \mathcal{B}_{i}} B\right) \leq N \boldsymbol{\alpha}(n) \sum_{\mathbf{B}(y, \sigma(y)) \in \mathcal{B}_{i}} \sigma(y)^{n} \\
& \leq N \boldsymbol{\alpha}(n) \frac{1}{2 \eta} \int_{\mathbf{C}}|\vec{T}-\overrightarrow{\mathbf{e}}|^{2} d\|T\|=N \boldsymbol{\alpha}(n) \frac{1}{\eta} \mathbf{E}(T, 1) .
\end{aligned}
$$

We want to show that spt $T$ is a graph over $G$. For this we fix $y, \tilde{y} \in G$ and $x, \tilde{x} \in \operatorname{spt} T$ with $\mathbf{p} x=y, \mathbf{p} \tilde{x}=\tilde{y}$, and we distinguish

Case 1: $\mathbf{B}(y, 2|y-\tilde{y}|) \subset \mathbf{U}_{+} \quad\left(\right.$ or $\left.\subset \mathbf{U}_{-}\right)$;

Case 2: $\mathbf{B}(y, 2|y-\tilde{y}|) \cap \mathbf{U}^{n-1} \times\{0\} \neq \emptyset$. 
In the first case we consider the scaled current $\widetilde{T}=\left(\left(\boldsymbol{\mu}_{2|y-\tilde{y}|}^{-1} \circ \boldsymbol{\tau}_{x}^{-1}\right)_{\#} T\right)\left\llcorner\mathbf{C} \in \mathcal{T}_{i}(n, k, m)\right.$ which is $(\widetilde{F}, \widetilde{\omega})$ minimizing in $\mathbf{C}$, where $\widetilde{F}$ has the same structure constant $\Lambda$ as $F$, and $\widetilde{\omega}(r)=\omega(2|y-\tilde{y}| r) \leq \omega(r)$ (see Section 1 ; in the case $y=\tilde{y}$ we consider $\left(\left(\boldsymbol{\mu}_{t}^{-1} \circ \boldsymbol{\tau}_{x}^{-1}\right)_{\#} T\right)\llcorner\mathbf{C}$ with $t>0$ arbitrary small.) We can therefore apply Lemma 2.2 (and Remark 2.3 in the case $m \geq 2$, as (2.13) for $T$ implies (2.12) for $\widetilde{T}$ ) to obtain

$$
|\mathbf{q} x-\mathbf{q} \tilde{x}| \leq \widetilde{C}_{3} \mathbf{E}(\widetilde{T}, 1)^{1 / 2 n} 2|y-\tilde{y}|
$$

provided $\widetilde{C}_{2} \mathbf{E}(\widetilde{T}, 1)^{1 / 2} \leq 1$. Here we have abbreviated $\widetilde{C}_{2}=C_{2}\left[\Lambda+\omega\left(\frac{1}{2}\right)\right]^{n-1}$ and $\widetilde{C}_{3}=$ $C_{3}^{1 / n}\left[\Lambda+\omega\left(\frac{1}{2}\right)\right]^{1-1 / n}$. From $\varrho \leq \frac{1}{5}$ we have $2|y-\tilde{y}|<1-\varrho$ and hence $\mathbf{E}(\widetilde{T}, 1)=$ $\mathbf{E}(T, y, 2|y-\tilde{y}|) \leq \eta$, by $(3.7)$. Choosing $\eta$ such that $\widetilde{C}_{2} \eta^{1 / 2} \leq 1$ the conclusion is therefore

$$
|\mathbf{q} x-\mathbf{q} \tilde{x}| \leq \widetilde{C}_{3} \eta^{1 / 2 n} 2|y-\tilde{y}| \quad \text { in case } 1 .
$$

If $\mathbf{B}(y, 2|y-\tilde{y}|) \subset \mathbf{U}_{-}$and $m \geq 2$, then $\tilde{T} \in \mathcal{T}_{i}(n, k, m-1)$, and the conclusion is the same.

In the second case we introduce $y^{\prime}=\left(y_{1}, \ldots, y_{n-1}, 0\right)$ in $\mathbb{R}^{n}$ and observe $\left|y^{\prime}-y\right| \leq 2|y-\tilde{y}|$, hence

$$
\mathbf{B}(y,|y-\tilde{y}|) \subset \mathbf{B}\left(y^{\prime}, 3|y-\tilde{y}|\right) \subset \mathbf{B}\left(y^{\prime}, 6|y-\tilde{y}|\right) \subset \mathbf{B}(y, 8|y-\tilde{y}|) \subset \mathbf{U},
$$

since $\varrho \leq \frac{1}{16}$. We now apply the height estimate form Section 2 to the scaled current $\widetilde{T}=\left(\left(\boldsymbol{\mu}_{6|y-\tilde{y}|}^{-1} \circ \boldsymbol{\tau}_{\left(y^{\prime}, 0\right)}^{-1}\right)_{\#} T\right)\left\llcorner\mathbf{C} \in \mathcal{T}_{b}(n, k, m)\right.$ and obtain

$$
\max \{|\mathbf{q} x|,|\mathbf{q} \tilde{x}|\} \leq \widetilde{C}_{3} \mathbf{E}\left(T, y^{\prime}, 6|y-\tilde{y}|\right)^{1 / 2 n} 6|y-\tilde{y}|,
$$

provided $\widetilde{C}_{2} \mathbf{E}\left(T, y^{\prime}, 6|y-\tilde{y}|\right)^{1 / 2} \leq 1$. On account of $\varrho \leq \frac{1}{17}$ we have $8|y-\tilde{y}|<1-\varrho$ and hence, by (3.7), $\mathbf{E}\left(T, y^{\prime}, 6|y-\tilde{y}|\right) \leq\left(\frac{4}{3}\right)^{n} \eta$. Choosing $\eta>0$ such that $\widetilde{C}_{2}\left(\frac{4}{3}\right)^{n / 2} \eta^{1 / 2} \leq 1$ we therefore obtain

$$
|\mathbf{q} x-\mathbf{q} \tilde{x}| \leq 2 \widetilde{C}_{3} \sqrt{\frac{4}{3}} \eta^{1 / 2 n} 6|y-\tilde{y}| \quad \text { in case } 2 .
$$

We also note

$$
|\mathbf{q} x| \leq \widetilde{C}_{3} \sqrt{\frac{3}{2}} \eta^{1 / 2 n} 2\left|y-y^{\prime}\right|
$$

which follws from the same reasoning if we apply it with $y^{\prime}$ instead of $\tilde{y}$ and use $\mathbf{B}\left(y,\left|y-y^{\prime}\right|\right)$ $\subset \mathbf{B}\left(y^{\prime}, 2\left|y-y^{\prime}\right|\right) \subset \mathbf{B}\left(y, 3\left|y-y^{\prime}\right|\right) \subset \mathbf{U}$.

Considering $y=\tilde{y}$ in (3.9), (3.10) we see that a function $g$ is well defined on $G$ in the case $m \geq 2$ and on $G \cap \mathbf{U}_{+}$in the case $m=1$ by letting $g(y)=\mathbf{q} x$ for $x \in \operatorname{spt} T$ with $\mathbf{p} x=y$. From (3.9), (3.10) we also see $\operatorname{Lip} g \leq \beta$ if we choose $\eta=C_{5}^{-1} \beta^{2 n}$ with $C_{5}=\operatorname{const}(n, m)\left[\Lambda+\omega\left(\frac{1}{2}\right)\right]^{2 n-2}$. From (3.11) we further recognize that this remains true if we extend $g$ to $\mathbf{U}_{\varrho}^{n-1} \times\{0\}$ by zero. Finally, we extend $g$ to all of $\mathbf{U}_{\varrho}$ preserving the Lipschitz constant, then $\operatorname{Lip} g \leq \beta \leq 1$ holds on $\mathbf{U}_{\varrho}$ and $(\mathrm{v})$ is valid. 
To prove (i) we note that $T_{g}$ is the $n$-current associated with graph $g$ and with multiplicities $m$ on $\mathbf{C}_{+} \cap \operatorname{graph} g, m-1$ on $\mathbf{C}_{-} \cap \operatorname{graph} g$, oriented such that $\left(\partial T_{g}\right)\left\llcorner\mathbf{C}_{\varrho}=(\partial T)\left\llcorner\mathbf{C}_{\varrho}\right.\right.$. Writing $G_{ \pm}=\mathbf{U}_{ \pm} \cap G$ and $A=\mathbf{U}_{\varrho} \backslash\left(G_{+} \cup G_{-}\right)$we estimate, using also (2.13) if $m>1$,

$$
\begin{aligned}
\left\|T-T_{g}\right\|\left(G_{+} \times \mathbb{R}^{k}\right) & =\int_{\text {graph }\left.g\right|_{G_{+}}}\left[\Theta^{n}(\|T\|, \cdot)-m\right] d \mathcal{H}^{n} \\
& \leq\|T\|\left(G_{+} \times \mathbb{R}^{k}\right)-m \mathcal{L}^{n}\left(G_{+}\right) \\
& =\mathbf{E}\left(T\left\llcorner\left(G_{+} \times \mathbb{R}^{k}\right), 1\right)\right.
\end{aligned}
$$

and simlarly

$$
\left\|T-T_{g}\right\|\left(G_{-} \times \mathbb{R}^{k}\right) \leq \mathbf{E}\left(T\left\llcorner\left(G_{-} \times \mathbb{R}^{k}\right), 1\right) \quad \text { in the case } m \geq 2,\right.
$$

while

$$
\|T\|\left(\mathbf{C} \backslash \mathbf{C}_{+}\right) \leq \mathbf{E}(T, 1) \quad \text { in the case } m=1
$$

because then $\left(\mathbf{p}_{\#} T\right)\left\llcorner\left(\mathbf{U} \backslash \mathbf{U}_{+}\right)=0\right.$. Furthermore,

$$
\begin{aligned}
\left\|T-T_{g}\right\| & \left(A \times \mathbb{R}^{k}\right) \leq\left(\|T\|+\left\|T_{g}\right\|\right)\left(A \times \mathbb{R}^{k}\right) \\
& \leq \mathbf{E}\left(T\left\llcorner\left(A \times \mathbb{R}^{k}\right), 1\right)+m\left[1+\left(1+(\operatorname{Lip} g)^{2}\right)^{n / 2}\right] \mathcal{L}^{n}(A) .\right.
\end{aligned}
$$

Adding (3.13), (3.14), (3.15), taking account of $\operatorname{Lip} g \leq \beta \leq 1$, and using also (3.8) which, by the choice of $\eta$ above, may be rewritten

$$
\mathcal{L}^{n}(A) \leq C_{5} N \boldsymbol{\alpha}(n) \beta^{-2 n} \mathbf{E}(T, 1)
$$

we deduce (3.1) in the case $m \geq 2$ writing $C_{5}$ for $C_{5} N \boldsymbol{\alpha}(n)$. In the case $m=1$ we obtain the same conclusion from (3.12),(3.14), (3.15), and (3.16), noting that $T_{g}\left\llcorner\left(\mathbf{C} \backslash \mathbf{C}_{+}\right)=0\right.$.

Assertion (ii) follows from (3.16) and (3.14), since $A_{g} \subset A \cup \mathbf{p}\left[\operatorname{spt}\left(T\left\llcorner\left(\mathbf{C} \backslash \mathbf{C}_{+}\right)\right)\right]\right.$if $m=1$.

For (iii) we use $|D g| \leq \operatorname{Lip} g \leq 1$, the definition of $A_{g}$ and (2.13) (if $m>1$ ) to infer

$$
\begin{aligned}
\int_{\mathbf{U}_{+} \cap \mathbf{U}_{\varrho} \backslash A_{g}}|D g|^{2} d \mathcal{L}^{n} & \leq 3 \int_{\mathbf{U}_{+} \cap \mathbf{U}_{\varrho} \backslash A_{g}}\left[\sqrt{1+|D g|^{2}}-1\right] d \mathcal{L}^{n} \\
& \leq 3 \int_{\mathbf{U}_{+} \cap \mathbf{U}_{\varrho} \backslash A_{g}}\left[J_{n}(\mathrm{id} \times g)-1\right] d \mathcal{L}^{n} \\
& \leq \frac{3}{m}\|T\|\left(\mathbf{p}^{-1}\left(\mathbf{U}_{+} \cap \mathbf{U}_{\varrho} \backslash A_{g}\right)\right)-3 \mathcal{L}^{n}\left(\mathbf{U}_{+} \cap \mathbf{U}_{\varrho} \backslash A_{g}\right) \\
& \leq \frac{3}{m} \mathbf{E}\left(T\left\llcorner\mathbf{C}_{+}, 1\right),\right.
\end{aligned}
$$

and a similar inequality, with $m$ replaced by $m-1$, holds for the integral on $\mathbf{U}_{-} \cap \mathbf{U}_{\varrho} \backslash A_{g}$ if $m \geq 2$.

With regard to (iv) we note that (iii) implies

$$
\mathcal{L}^{n}\left(A_{g, \gamma}\right) \leq\left(1+\frac{1}{\gamma^{2}}\right) \mathcal{L}^{n}\left(A_{g}\right)+\frac{3}{\gamma^{2}} \mathbf{E}(T, 1)
$$


hence (3.4) follows from (3.2), and (3.5) from

$$
\|T\|\left(\mathbf{p}^{-1} A_{g, \gamma}\right) \leq \mathbf{E}(T, 1)+m \mathcal{L}^{n}\left(A_{g, \gamma}\right) .
$$

Finally, (vi) follows from Lemma 2.2, because $g$ can be chosen to satisfy (3.6) if we secure $\widetilde{C}_{2} \mathbf{E}(T, 1)^{1 / 2} \leq 1$.

For the next lemma we introduce

$$
A=\left(a_{i j}^{\alpha \beta}\right)=\left(\frac{\partial^{2}}{\partial p_{\alpha}^{i} \partial p_{\beta}^{j}} F_{0}^{\S}(0)\right)
$$

for the Hessean matrix of the nonparametric integrand $F_{0}^{\S}(p)$ associated with the frozen integrand $F_{0}=F(0, \cdot)$. We also write

$$
A(\xi, \eta)=\sum_{\alpha, \beta=1}^{n} \sum_{i, j=1}^{k} a_{i j}^{\alpha \beta} \xi_{\alpha}^{i} \eta_{\beta}^{j}
$$

for the quadratic form on $\operatorname{Hom}\left(\mathbb{R}^{n}, \mathbb{R}^{k}\right)$ associated with $A$, i.e. $A=D^{2} F_{0}^{\S}(0)$. The constant $\Lambda$ and the nondecreasing functions $\kappa, \mu, \widehat{\mu}$, and $\nu$ are from the structure conditions (1.1)(1.9) on $F$.

3.2 Lemma (approximate $\boldsymbol{A}$-harmonicity). Suppose $\omega\left(\frac{1}{2}\right)+\mu\left(\frac{1}{2}\right) \leq 1,0<\varrho \leq \frac{1}{17}$, $\mathbf{C}_{\varrho} \cap \operatorname{spt} T \subset \mathbf{U}_{\varrho}^{n} \times \mathbf{B}_{1 / 3}^{k}$, and let $g$ be the Lipschitz approximation associated with $T$, $\varrho$, and $\beta=1$ by Lemma 3.1. Then, with $C_{10}=\operatorname{const}(n, m, \Lambda), E=\mathbf{E}(T, 1)$, and $\chi=$ $\kappa\left(\frac{1}{2}\right)^{2}+\widehat{\mu}\left(\frac{1}{2}\right)+\omega\left(\frac{1}{2}\right)$, the inequality

$$
\begin{aligned}
& \left|\int_{\mathbf{U}_{\varrho}} A(D g, D \zeta) d \mathcal{L}^{n}\right| \\
& \quad \leq C_{10}\left[\gamma^{-2} E+\sqrt{\chi}(1+E)+(\sqrt{E}+\sqrt{\chi}) \nu(\gamma+\sqrt{\chi})\right] \sup |D \zeta|
\end{aligned}
$$

holds for all parameters $0<\gamma \leq 1$ and all functions $\zeta \in C^{1}\left(\mathbf{U}, \mathbb{R}^{k}\right)$ with compact support in $\mathbf{U}_{\varrho}$ and satisfying additionally $\operatorname{spt} \zeta \subset \mathbf{U}_{+}$in the case $T \in \mathcal{T}_{b}(n, k, 1)$, spt $\zeta \subset \mathbf{U} \backslash \mathbf{U}^{n-1} \times\{0\}$ in the case $T \in \mathcal{T}_{b}(n, k, m), m \geq 2$.

The essential point here is that $\chi / \sqrt{E}$ is small in the later application of this lemma so that, with the appropriate choice of $\gamma$, the constant on the right hand side of (3.17) is a small quantity times $\sqrt{E}$. Since $\widehat{\mu}\left(\frac{1}{2}\right) \leq \mu\left(\frac{1}{2}\right)$ we could replace $\widehat{\mu}\left(\frac{1}{2}\right)$ by $\mu\left(\frac{1}{2}\right)$ in the hypothesis. However, replacing $\widehat{\mu}\left(\frac{1}{2}\right)$ by $\mu\left(\frac{1}{2}\right)$ in the conclusion, i.e. in the definition of $\chi$, would yield a weaker conclusion, in general. The hypothesis that $T$ has height $\leq \frac{1}{3}$ in $\mathbf{C}_{\varrho}$ is made only to secure that the $(F, \omega)$ minimizing property of $T$ is applied in the proof with a modification of $T$ inside a ball of radius $\frac{1}{2}$. An appropriate version of the lemma is true without this hypothesis.

Proof. We follow [Bo, Section IV] closely. We may assume sup $|D \zeta| \leq 1$, sup $|\zeta| \leq \varrho$, and we define the vertical deformation

$$
h_{t}(x)=x+t(0, \zeta(\mathbf{p} x)) \text { for }|t| \leq 1
$$


We then decompose

$$
\mathbf{F}\left(\left(h_{t \#} T\right)\left\llcorner\mathbf{C}_{\varrho}\right)=K(t)+L(t)+M(t)\right.
$$

where

$$
\begin{aligned}
& K(t)=\int_{A_{\gamma} \times \mathbb{R}^{k}} F\left(h_{t}(x), \bigwedge_{n} D h_{t}(x) \vec{T}(x)\right) d\|T\| x, \\
& L(t)=\int_{\mathbf{C}_{\varrho} \backslash A_{\gamma} \times \mathbb{R}^{k}} F\left(h_{t}(x), \bigwedge_{n} D h_{t}(x) \vec{S}_{g}(x)\right) d\left\|S_{g}\right\| x, \\
& M(t)=\int_{\mathbf{C}_{\varrho} \backslash A_{\gamma} \times \mathbb{R}^{k}} F\left(h_{t}(x), \bigwedge_{n} D h_{t}(x) \vec{T}_{g}(x)\right) d\left\|T_{g}\right\| x
\end{aligned}
$$

and we have written $A_{\gamma}$ for the set $A_{g, \gamma} \subset \mathbf{U}_{\varrho}$ defined in Lemma 3.1, (iv) and $S_{g}$ for $T-T_{g}$. (Note that $T-T_{g}=T\left\llcorner\vartheta\right.$ in $\mathbf{C}_{\varrho} \backslash A_{\gamma} \times \mathbb{R}^{k}$ with a nonnegative function $\vartheta$, cf. (3.13).)

We estimate $K(s)-K(0)$ using the continuity modulus $\mu(\bullet)$ for $x \mapsto F(x, \xi)$ from (1.7) and the bound $\Lambda$ for the derivative $D_{(2)} F$ from (1.2). We have, for $|s| \leq 1$, and $\|T\|$ almost all $x \in \mathbf{C}_{\varrho}$,

$$
\begin{aligned}
& \left|F\left(h_{s}(x), \bigwedge_{n} D h_{s}(x) \vec{T}(x)\right)-F(x, \vec{T}(x))\right| \\
& \leq\left|F\left(h_{s}(x), \bigwedge_{n} D h_{s}(x) \vec{T}(x)\right)-F\left(x, \bigwedge_{n} D h_{s}(x) \vec{T}(x)\right)\right| \\
& +\left|\int_{0}^{s} D_{(2)} F\left(x, \bigwedge_{n} D h_{t}(x) \vec{T}(x)\right) \frac{d}{d t} \bigwedge_{n} D h_{t}(x) \vec{T}(x) d t\right| \\
& \leq \mu(|s \zeta(\mathbf{p} x)|)\left|\bigwedge_{n} D h_{s}(x) \vec{T}(x)\right|+\Lambda\left|\int_{0}^{s}\right| \frac{d}{d t} \bigwedge_{n} D h_{t}(x) \vec{T}(x)|d t| \\
& \leq \mu(|s| \varrho) 2^{n}+|s| \Lambda n 2^{n-1} .
\end{aligned}
$$

Integrating with respect to $\|T\|\left\llcorner A_{\gamma} \times \mathbb{R}^{k}\right.$ we obtain

$$
|K(s)-K(0)| \leq\left(\mu(|s| \varrho) 2^{n}+|s| \Lambda n 2^{n-1}\right)\|T\|\left(A_{\gamma} \times \mathbb{R}^{k}\right) \leq C_{6}(\mu(|s| \varrho)+|s|) \gamma^{-2} E,
$$

where $C_{6}=\operatorname{const}(n, m, \Lambda)$ and we have used (3.5).

In the same way, using (3.1) instead of (3.5), we derive

$$
|L(s)-L(0)| \leq C_{7}(\mu(|s| \varrho)+|s|) E
$$

with $C_{7}=\operatorname{const}(n, m, \Lambda)$.

Similarly as above we see, using also the continuity modulus $\kappa(\bullet)$ for $x \mapsto D_{(2)} F(x, \xi)$ from (1.6) and again assuming $|s| \leq 1$,

$$
\begin{aligned}
& \left|F\left(h_{s}(x), \bigwedge_{n} D h_{s}(x) \vec{T}_{g}(x)\right)-F\left(x, \vec{T}_{g}(x)\right)-F\left(0, \bigwedge_{n} D h_{s}(x) \vec{T}_{g}(x)\right)+F\left(0, \vec{T}_{g}(x)\right)\right| \\
& \leq\left|F\left(h_{s}(x), \bigwedge_{n} D h_{s}(x) \vec{T}_{g}(x)\right)-F\left(x, \bigwedge_{n} D h_{s}(x) \vec{T}_{g}(x)\right)\right|
\end{aligned}
$$




$$
\begin{aligned}
& +\left|\int_{0}^{s}\left[D_{(2)} F\left(x, \bigwedge_{n} D h_{t}(x) \vec{T}_{g}(x)\right)-D_{(2)} F\left(0, \bigwedge_{n} D h_{t}(x) \vec{T}_{g}(x)\right)\right] \frac{d}{d t} \bigwedge_{n} D h_{t}(x) \vec{T}_{g}(x) d t\right| \\
& \leq \mu(|s| \varrho) 2^{n}+|s| \kappa(|x|) n 2^{n-1}
\end{aligned}
$$

Integrating this with respect to $\left\|T_{g}\right\|\left\llcorner\left(\mathbf{C}_{\varrho} \backslash A_{\gamma} \times \mathbb{R}^{k}\right)\right.$ we get

$$
\begin{aligned}
\mid \int_{\mathbf{C}_{\varrho} \backslash A_{\gamma} \times \mathbb{R}^{k}}[ & \left.F\left(h_{s}(x), \bigwedge_{n} D h_{s}(x) \vec{T}_{g}(x)\right)-F\left(x, \vec{T}_{g}(x)\right)\right] d\left\|T_{g}\right\| x \\
& -\int_{\mathbf{C}_{\varrho} \backslash A_{\gamma} \times \mathbb{R}^{k}}\left[F\left(0, \bigwedge_{n} D h_{s}(x) \vec{T}_{g}(x)\right)-F\left(0, \vec{T}_{g}(x)\right)\right] d\left\|T_{g}\right\| x \mid \\
\leq & \operatorname{const}(n, m)[\mu(|s| \varrho)+|s| \kappa(\sqrt{2} \varrho)],
\end{aligned}
$$

since Lip $g \leq 1$ implies $\left\|T_{g}\right\|\left(\mathbf{C}_{\varrho}\right) \leq m \operatorname{const}(n)$ and $|x| \leq \sqrt{2} \varrho$ for $x \in \mathbf{C}_{\varrho} \cap \operatorname{spt} T_{g}$ (recall $g(0)=0)$.

Defining the multiplicity function $\boldsymbol{\iota}_{m}$ by $\boldsymbol{\iota}_{m} \equiv m$ on $\mathbf{U}$ in the case $T \in \mathcal{T}_{i}(n, k, m)$ and $\iota_{m} \equiv m$ on $\mathbf{U}_{+}, \boldsymbol{\iota}_{m} \equiv m-1$ on $\mathbf{U}_{-}$in the case $T \in \mathcal{T}_{b}(n, k, m)$ we can write

$$
\int_{\mathbf{C}_{\varrho} \backslash A_{\gamma} \times \mathbb{R}^{k}} F\left(0, \bigwedge_{n} D h_{s}(x) \vec{T}_{g}(x)\right) d\left\|T_{g}\right\| x=\int_{\mathbf{U}_{\varrho} \backslash A_{\gamma}} F_{0}^{\S}(D(g+s \zeta)) \iota_{m} d \mathcal{L}^{n}
$$

and

$$
\int_{\mathbf{C}_{\varrho} \backslash A_{\gamma} \times \mathbb{R}^{k}} F\left(0, \vec{T}_{g}(x)\right) d\left\|T_{g}\right\| x=\int_{\mathbf{U}_{\varrho} \backslash A_{\gamma}} F_{0}^{\S}(D g) \iota_{m} d \mathcal{L}^{n} .
$$

Using (1.11) in the Taylor expansion

$$
\left\|D F_{0}^{\S}(p)-D F_{0}^{\S}(0)-D D F_{0}^{\S}(0) p\right\| \leq \nu(|p|)|p|
$$

with $p=D g(y)+t D \zeta(y)$, multiplying with $D \zeta(y)$, and integrating with respect to $(t, y)$ we further obtain

$$
\begin{aligned}
& \mid \int_{\mathbf{U}_{\varrho} \backslash A_{\gamma}}\left[F_{0}^{\S}(D(g+s \zeta))-F_{0}^{\S}(D g)\right] \iota_{m} d \mathcal{L}^{n}-s \int_{\mathbf{U}_{\varrho} \backslash A_{\gamma}} D F_{0}^{\S}(0) D \zeta \iota_{m} d \mathcal{L}^{n} \\
& \quad-s \int_{\mathbf{U}_{\varrho} \backslash A_{\gamma}} D^{2} F_{0}^{\S}(0)(D g, D \zeta) \boldsymbol{\iota}_{m} d \mathcal{L}^{n}-\frac{1}{2} s^{2} \int_{\mathbf{U}_{\varrho} \backslash A_{\gamma}} D^{2} F_{0}^{\S}(0)(D \zeta, D \zeta) \iota_{m} d \mathcal{L}^{n} \mid \\
& \quad \leq\left|\int_{0}^{s} \int_{\mathbf{U}_{\varrho} \backslash A_{\gamma}}(\nu \circ|D g+t D \zeta|)\right| D g+t D \zeta|| D \zeta\left|\iota_{m} d \mathcal{L}^{n} d t\right| \\
& \quad \leq m \operatorname{const}(n)|s| \nu(\gamma+|s|)(\sqrt{E}+|s|)
\end{aligned}
$$

where we have used (3.3), $|D \zeta| \leq 1$ and $|D g| \leq \gamma$ on $\mathbf{U}_{\varrho} \backslash A_{\gamma}$ (on $\mathbf{U}_{+} \cap \mathbf{U}_{\varrho} \backslash A_{\gamma}$ in the case $\left.T \in \mathcal{T}_{b}(n, k, 1)\right)$ in the last step. The second integral appearing in (3.23) can be estimated 
with (3.4), because the integral of $D F_{0}^{\S}(0) D \zeta \iota_{m}$ on $\mathbf{U}_{\varrho}$ vanishes on account of the hypothesis on $\operatorname{spt} \zeta$ :

$$
\left|\int_{\mathbf{U}_{\varrho} \backslash A_{\gamma}} D F_{0}^{\S}(0) D \zeta \iota_{m} d \mathcal{L}^{n}\right|=\left|\int_{A_{\gamma}} D F_{0}^{\S}(0) D \zeta \iota_{m} d \mathcal{L}^{n}\right| \leq \operatorname{const}(n, m, \Lambda) \gamma^{-2} E .
$$

Combining (3.20)-(3.24) and applying (3.4) once more we arrive finally at the following inequality, valid for $|s| \leq 1$ :

$$
\begin{aligned}
\mid M(s)- & M(0)-s \int_{\mathbf{U}_{\varrho}} D^{2} F_{0}^{\S}(0)(D g, D \zeta) \iota_{m} d \mathcal{L}^{n} \mid \\
\leq & C_{8} \mu(|s| \varrho)+C_{8}|s|\left[\kappa(\sqrt{2} \varrho)+\gamma^{-2} E+|s|+(\sqrt{E}+|s|) \nu(\gamma+|s|)\right],
\end{aligned}
$$

where $C_{8}=\operatorname{const}(n, m, \Lambda)$. With $(3.18),(3.19),(3.25)$, and the inequality $\widehat{\mu}\left(\frac{1}{2}\right) \geq \mu(|s| \varrho)-$ $4 s^{2} \varrho^{2}$ which follows from $(1.9)$, we have proved

$$
\begin{gathered}
\mid \mathbf{F}\left(\left(h_{s \#} T\right)\left\llcorner\mathbf{C}_{\varrho}\right)-\mathbf{F}\left(T\left\llcorner\mathbf{C}_{\varrho}\right)-s \int_{\mathbf{U}_{\varrho}} D^{2} F_{0}^{\S}(0)(D g, D \zeta) \iota_{M} d \mathcal{L}^{n} \mid\right.\right. \\
\leq C_{9} \mu(|s| \varrho)+C_{9}|s|\left[\kappa(\sqrt{2} \varrho)+\gamma^{-2} E+|s|+(\sqrt{E}+|s|) \nu(\gamma+|s|)\right] \\
\leq C_{9} \widehat{\mu}\left(\frac{1}{2}\right)+C_{9}|s|\left[\kappa\left(\frac{1}{2}\right)+\gamma^{-2} E+2|s|+(\sqrt{E}+|s|) \nu(\gamma+|s|)\right]
\end{gathered}
$$

provided $|s| \leq 1$ and $\operatorname{spt} \zeta$ is compact in $\mathbf{U}_{\varrho}$ not meeting $\mathbf{U}^{n-1} \times\{0\}$ unless $\boldsymbol{\iota}_{m}$ is constant.

Since, with this condition on $\operatorname{spt} \zeta, h_{s \#} T$ is an admissible comparison current, we can employ the $(F, \omega)$ minimality of $T$ :

$$
\begin{aligned}
\mathbf{F}\left(T\left\llcorner\mathbf{C}_{\varrho}\right)\right. & \leq \mathbf{F}\left(\left(h_{s \#} T\right)\left\llcorner\mathbf{C}_{\varrho}\right)+\omega\left(\frac{1}{2}\right) \mathbf{M}\left(h_{s \#} T\left\llcorner\mathbf{C}_{\varrho}\right)\right.\right. \\
& \leq \mathbf{F}\left(\left(h_{s \#} T\right)\left\llcorner\mathbf{C}_{\varrho}\right)+\omega\left(\frac{1}{2}\right) \operatorname{const}(n)(m+E),\right.
\end{aligned}
$$

because $h_{s \#} T-T$ has support in $\mathbf{B}^{n+k}\left(0, \frac{1}{2}\right)$, by our assumptions on $\mathbf{C}_{\varrho} \cap \operatorname{spt} T, \zeta$ and $\varrho$. Choosing $|s|=\sqrt{\widehat{\mu}\left(\frac{1}{2}\right)+\omega\left(\frac{1}{2}\right)}$ (we may assume that this is positive) and the sign of $s$ carefully (as in [Bo], proof of Lemma 13) we deduce (3.17) from (3.26) and (3.27). The condition $|s| \leq 1$ is satisfied for this choice in view of the hypothesis $\mu\left(\frac{1}{2}\right)+\omega\left(\frac{1}{2}\right) \leq 1$ (and $\left.\widehat{\mu}\left(\frac{1}{2}\right) \leq \mu\left(\frac{1}{2}\right)\right)$.

For $T \in \mathcal{T}_{b}(n, k, m)$ with multiplicity $m \geq 2$ it is reasonable to expect that the condition $\mathbf{U}^{n-1} \times\{0\} \cap \operatorname{spt} \zeta=\emptyset$ in (3.17) is not necessary, i.e. $g$ is approximately $A$-harmonic on $\mathbf{U}_{\varrho}$ and not just on $\mathbf{U}_{+} \cap \mathbf{U}_{\varrho}$ and $\mathbf{U}_{-} \cap \mathbf{U}_{\varrho}$ separately. However, we could not find a proof based on vertical deformations of the type used above, and this is one of the reasons why we can obtain a boundary regularity result only in the case $m=1$. It seems that a different type of variations of $T$ is needed to make progress here, variations which can separate "leaves" of $T$ with zero boundary in $\mathbf{C}$ from the "leaf" with boundary $\partial T$ and multiplicity 1 , speaking intuitively.

So far we have not used ellipticity of the integrand $F$. However, if $A=D^{2} F_{0}^{\S}(0)$ is elliptic in the sense of the Legendre-Hadamard condition (1.13), then we can find an 
$\boldsymbol{A}$-harmonic function, i.e. an exact solution to the homogeneous constant coefficient elliptic system associated with $A$, which is close in the weak topology of the Sobolev space $W^{1,2}$, to the approximatively $A$-harmonic function $g$ exhibited in Lemma 3.2. This can be proved similarly as in [Si1, §21], [Si2, §1]. For the convenience of the reader we formulate in the next lemma the exact statement which will be used later, and we include the simple proof.

3.3 Lemma ( $\boldsymbol{A}$-harmonic approximation). There exists a positive function $\delta(n, k, \lambda$, $\Lambda, \epsilon) \leq 1$ with the following property: Whenever $A \in \bigodot^{2} \operatorname{Hom}\left(\mathbb{R}^{n}, \mathbb{R}^{k}\right)$ is (rank one) elliptic with ellipticity constant $\lambda>0$ and upper bound $\Lambda, \epsilon$ and $\varrho$ are given positive numbers, and $f \in W^{1,2}\left(\mathbf{U}_{\varrho}, \mathbb{R}^{k}\right)$ with $\varrho^{2-n} \int_{\mathbf{U}_{\varrho}}|D f|^{2} d \mathcal{L}^{n} \leq 1$ is approximatively A-harmonic in the sense that

$$
\left|\varrho^{2-n} \int_{\mathbf{U}_{\varrho}} A(D f, D \zeta) d \mathcal{L}^{n}\right| \leq \delta(n, k, \lambda, \Lambda, \epsilon) \varrho \sup |D \zeta|
$$

holds for all $\zeta \in C^{1}\left(\mathbf{U}_{\varrho}, \mathbb{R}^{k}\right)$ with compact support in $\mathbf{U}_{\varrho}$, then there exists an A-harmonic function $u \in W^{1,2}\left(\mathbf{U}_{\varrho}, \mathbb{R}^{k}\right)$ such that

$$
\varrho^{-n} \int_{\mathbf{U}_{\varrho}}|u-f|^{2} d \mathcal{L}^{n} \leq \epsilon \quad \text { and } \quad \varrho^{2-n} \int_{\mathbf{U}_{\varrho}}|D u|^{2} d \mathcal{L}^{n} \leq 1
$$

The same assertion is true if we replace, everywhere in the statement, the $n$-ball $\mathbf{U}_{\varrho}$ by the half ball $\left.\mathbf{U}_{\varrho,+}=\mathbf{U}_{\varrho} \cap \mathbb{R}^{n-1} \times\right] 0, \infty\left[\right.$ and the Sobolev space $W^{1,2}\left(\mathbf{U}_{\varrho}, \mathbb{R}^{k}\right)$ by $W_{(0)}^{1,2}\left(\mathbf{U}_{\varrho,+}, \mathbb{R}^{k}\right)$, the space of $W^{1,2}$ functions on $\mathbf{U}_{\varrho,+}$ with vanishing boundary trace on $\mathbf{U}_{\varrho}^{n-1} \times\{0\}$.

Proof. We may assume $\varrho=1$, the general case follows by scaling $y \mapsto \varrho^{-1} f(\varrho y)$. Supposing the Lemma to be false we have existence of a sequence of quadratic forms $A_{i} \in$ $\bigodot^{2} \operatorname{Hom}\left(\mathbb{R}^{n}, \mathbb{R}^{k}\right)$ with fixed ellipticity constant $\lambda>0$ and bound $\Lambda<\infty$, and a sequence of $f_{i} \in W^{1,2}\left(\mathbf{U}, \mathbb{R}^{k}\right)$ with $\left\|D f_{i}\right\|_{L^{2}} \leq 1$ and

$$
\left|\int_{\mathbf{U}} A_{i}\left(D f_{i}, D \zeta\right) d \mathcal{L}^{n}\right| \leq \frac{1}{i} \sup |D \zeta|
$$

for all $\zeta \in C^{1}\left(\mathbf{U}, \mathbb{R}^{k}\right)$ with compact support in $\mathbf{U}$, such that for some $\epsilon>0$ the inequality

$$
\int_{\mathbf{U}}\left|f_{i}-u_{i}\right|^{2} d \mathcal{L}^{n}>\epsilon
$$

is valid for each $i$ and each $A_{i}$-harmonic function $u_{i} \in W^{1,2}\left(\mathbf{U}, \mathbb{R}^{k}\right)$ with $\left\|D u_{i}\right\|_{L^{2}} \leq 1$.

Clearly, we may assume $\int_{\mathbf{U}} f_{i} d \mathcal{L}^{n}=0$ and apply Poincaré's inequality and Rellich's theorem to obtain, after having passed to a subsequence, $f_{i} \rightarrow f$ strongly in $L^{2}\left(\mathbf{U}, \mathbb{R}^{k}\right)$ and weakly in $W^{1,2}\left(\mathbf{U}, \mathbb{R}^{k}\right)$ and $A_{i} \rightarrow A$ in $\bigodot^{2} \operatorname{Hom}\left(\mathbb{R}^{n}, \mathbb{R}^{k}\right)$. Then $\|D f\|_{L^{2}} \leq 1$, and from

$$
\begin{aligned}
\int_{\mathbf{U}} A(D f, D \zeta) d \mathcal{L}^{n}=\int_{\mathbf{U}} A(D f & \left.-D f_{i}, D \zeta\right) d \mathcal{L}^{n} \\
& +\int_{\mathbf{U}}\left(A-A_{i}\right)\left(D f_{i}, D \zeta\right) d \mathcal{L}^{n}+\int_{\mathbf{U}} A_{i}\left(D f_{i}, D \zeta\right) d \mathcal{L}^{n}
\end{aligned}
$$

and (3.28) we infer that $f$ is $A$-harmonic. 
Now, the ellipticity of $A_{i}$ implies (by Fourier transform and Plancherels theorem, i.e. Gårdings inequality in the case of constant coefficients) the coercivity condition

$$
\int_{\mathbf{U}} A_{i}(D \eta, D \eta) d \mathcal{L}^{n} \geq \lambda \int_{\mathbf{U}}|D \eta|^{2} d \mathcal{L}^{n} \quad \text { for } \eta \in W_{0}^{1,2}\left(\mathbf{U}, \mathbb{R}^{k}\right),
$$

hence we can choose $u_{i}$ as the solution to the Dirichlet problem corresponding to $A_{i}$ and the boundary values of $f$, i.e.

$$
u_{i}-f \in W_{0}^{1,2}\left(\mathbf{U}, \mathbb{R}^{k}\right), \quad \int_{\mathbf{U}} A_{i}\left(D u_{i}, D \zeta\right) d \mathcal{L}^{n}=0 \quad \text { for } \eta \in W_{0}^{1,2}\left(\mathbf{U}, \mathbb{R}^{k}\right)
$$

Then

$$
\begin{aligned}
\lambda \int_{\mathbf{U}}\left|D u_{i}-D f\right|^{2} d \mathcal{L}^{n} & \leq \int_{\mathbf{U}} A_{i}\left(D u_{i}-D f, D u_{i}-D f\right) d \mathcal{L}^{n} \quad \text { by }(3.30), \\
& =-\int_{\mathbf{U}} A_{i}\left(D f, D u_{i}-D f\right) d \mathcal{L}^{n} \quad \text { by }(3.31), \\
& =\int_{\mathbf{U}}\left(A-A_{i}\right)\left(D f, D u_{i}-D f\right) d \mathcal{L}^{n} \quad \text { by } A \text {-harmonicity of } f, \\
& \leq\left|A_{i}-A\right|\left[\int_{\mathbf{U}}\left|D u_{i}-D f\right|^{2} d \mathcal{L}^{n}\right]^{1 / 2} \quad \text { on account of }\|D f\|_{L^{2}} \leq 1,
\end{aligned}
$$

and in view of $A_{i} \rightarrow A$ we have convergence $u_{i} \rightarrow f$ in $W^{1,2}$ norm. But this implies $\left\|u_{i}-f_{i}\right\|_{L^{2}} \leq\left\|u_{i}-f\right\|_{L^{2}}+\left\|f-f_{i}\right\|_{L^{2}} \rightarrow 0$ as $i \rightarrow \infty$ and the same assertion is true for $\tilde{u}_{i}=\min \left(1,\left\|D u_{i}\right\|_{L^{2}}^{-1}\right) u_{i}$ contradicting (3.29).

The proof for $W_{(0)}^{1,2}\left(\mathbf{U}_{+}, \mathbb{R}^{k}\right)$ is the same. We need only to observe that Poincaré's inquality is valid in $W_{(0)}^{1,2}\left(\mathbf{U}_{+}, \mathbb{R}^{k}\right)$, Rellich's compactness theorem holds for the embedding $W_{(0)}^{1,2}\left(\mathbf{U}_{+}, \mathbb{R}^{k}\right) \rightarrow L^{2}\left(\mathbf{U}_{+}, \mathbb{R}^{k}\right)$, and $W_{(0)}^{1,2}\left(\mathbf{U}_{+}, \mathbb{R}^{k}\right)$ is a closed subspace of $W^{1,2}\left(\mathbf{U}_{+}, \mathbb{R}^{k}\right)$.

Although the proof of the previous lemma is by contradiction, this does not mean that our regularity proof is indirect. Our point of view is simply that the lemma guarantees the existence of a positive function $\delta(n, k, \lambda, \Lambda, \epsilon)$ which is, admittedly not in an explicit form, determined by a property of constant coefficient elliptic systems, and we will later use this function in the regularity proof.

\section{A reverse Poincaré inequality}

For solutions $u$ to an elliptic equation by a reverse Poincaré inequality (also called Caccioppoli's inequality) one means an estimate of Dirichlet's integral of $u$ (on a ball) in terms of the integral of $|u|^{2}$ (on a larger ball). The analogue for $(F, \omega)$ minimizing currents $T \in \mathcal{T}_{*}(n, k, m)$ would be an estimate of the excess $\mathbf{E}\left(T, \frac{1}{5}\right)$ of $T$ in $\mathbf{C}_{1 / 5}$, say, by the integral $\int_{\mathbf{C}}|\mathbf{q} x|^{2} d\|T\|$ of the squared height function. Up to some small extra term we prove here such an inequality. In Lemma 4.1 we first establish a weak version which has an additional term $\vartheta \mathbf{E}(T, 1)$ on the right-hand side, with an arbitrarily small parameter $\vartheta>0$, however. The proof is a refinement of [Bo, Sec. 5] which requires some new estimates; this is crucial for the optimality of our regularity results. We then apply a covering argument which we have found in [Si2] to deduce the strong form of the reverse Poincaré inequality in Lemma 4.2. In the following we use the ellipticity constant $\lambda>0$ of the integrand $F$ from (1.12), the bound $\Lambda$ from the structure conditions (1.1)-(1.3) for $F$, and the moduli $\kappa, \mu, \widehat{\mu}$ for $F$ from $(1.5)-(1.9)$. 
4.1 Lemma (weak reverse Poincaré inequality). Suppose $T \in \mathcal{T}_{i}(n, k, m)$ with $m \geq 0$ or $T \in \mathcal{T}_{b}(n, k, m)$ with $m \geq 1$ is $(F, \omega)$ minimizing in $\mathbf{C}$ and $\mathbf{C}_{7 / 8} \cap \operatorname{spt} T$ is contained in the ball $\mathbf{B}^{n+k}(0, R)$. Then, with $\chi(R)=\kappa(R)^{2}+\widehat{\mu}(R)+\omega(R)$ the following inequality holds for all parameters $\vartheta>0$,

$$
\mathbf{E}\left(T, \frac{1}{2}\right) \leq \vartheta \mathbf{E}(T, 1)+\frac{1}{\vartheta} C_{11}\left[\chi(R) \mathbf{M}(T)+(1+\chi(R)) \int_{\mathbf{C}}|\mathbf{q} x|^{2} d\|T\| x\right],
$$

where $C_{11}=\operatorname{const}(n, \lambda, \Lambda)$.

Here $\mathcal{T}_{i}(n, k, 0)$ is defined exactly as $\mathcal{T}_{i}(n, k, m)$ for $m \geq 1$ in Section 1 , whithout the condition $0 \in \operatorname{spt} T$, however. It will be clear from the proof that this latter condition is not used in the case $T \in \mathcal{T}_{i}(n, k, m)$ with $m \geq 1$.

Proof. For $0<r \leq 1$ we write $T_{r}=T\left\llcorner\mathbf{C}_{r}\right.$ and $\langle T, r\rangle=\partial T_{r}-(\partial T)\left\llcorner\mathbf{C}_{r}\right.$ for the cylindrical slices of $T$. Recalling the multiplicity function $\iota_{m} \equiv m$ on $\mathbf{U}$ in the case $T \in$ $\mathcal{T}_{i}(n, k, m)$ and $\boldsymbol{\iota}_{m} \equiv m$ on $\mathbf{U}_{+}, \boldsymbol{\iota}_{m} \equiv m-1$ on $\mathbf{U}_{-}$in the case $T \in \mathcal{T}_{b}(n, k, m)$ we further denote by $P_{r}$ the image of $\left(\mathbf{p}_{\#} T\right)\left\llcorner\mathbf{U}_{r}=\left(\mathbf{E}^{n}\left\llcorner\mathbf{U}_{r}\right)\left\llcorner\iota_{m}\right.\right.\right.$ under the inclusion $\mathbb{R}^{n} \rightarrow \mathbb{R}^{n} \times \mathbb{R}^{k}$. Using, for $0<\vartheta \leq \frac{5}{8}$ and $r>\frac{1}{5}$, the homotopy

$$
h^{\vartheta}(t, x)=x-t(\vartheta \mathbf{p} x, \mathbf{q} x)=(1-t) x+t(1-\vartheta)(\mathbf{p} x, 0)
$$

we define, similarly as in [Bo, Section V],

$$
\Sigma_{r, \vartheta}=P_{(1-\vartheta) r}-h_{\#}^{\vartheta}(\llbracket 0,1 \rrbracket \times\langle T, r\rangle)
$$

and verify

$$
\begin{aligned}
& \Sigma_{r, \vartheta}=\Sigma_{r, \vartheta}\left\llcorner\mathbf{C}_{r}, \quad \Sigma_{r, \vartheta}\left\llcorner\mathbf{C}_{1 / 2}=P_{1 / 2},\right.\right. \\
& \mathbf{p}_{\#} \Sigma_{r, \vartheta}=\mathbf{p}_{\#} T_{r}=\left(\mathbf { E } ^ { n } \llcorner \mathbf { U } _ { r } ) \left\llcorner\iota_{m}, \quad \partial \Sigma_{r, \vartheta}=\partial T_{r},\right.\right.
\end{aligned}
$$

so that $\Sigma_{r, \vartheta}$ may be used as a comparison current for $T_{r}$ if $\langle T, r\rangle$ is rectifiable.

For such $r$ we estimate the excess of $\Sigma_{r, \vartheta}$ with the standard estimate [Fe, 4.1.9] noting $\operatorname{Lip} h^{\vartheta}(t, \cdot) \leq 1-t \vartheta$ :

$$
\begin{aligned}
\mathbf{E}\left(\Sigma_{r, \vartheta}, 1\right) & =\mathbf{M}\left(P_{(1-\vartheta) r}\right)+\mathbf{M}\left(h_{\#}^{\vartheta}(\llbracket 0,1 \rrbracket \times\langle T, r\rangle)\right)-\mathbf{M}\left(P_{r}\right) \\
& \leq\left[(1-\vartheta)^{n}-1\right] \mathbf{M}\left(P_{r}\right)+\int_{0}^{1} \int_{\partial \mathbf{C}_{r}}|(\vartheta \mathbf{p} x, \mathbf{q} x)|(1-t \vartheta)^{n-1} d\|\langle T, r\rangle\| x d t \\
& \leq\left[1-(1-\vartheta)^{n}\right]\left[\frac{1}{n \vartheta} \int_{\partial \mathbf{C}_{r}}\left(\vartheta r+\frac{1}{2 \vartheta r}|\mathbf{q} x|^{2}\right) d\|\langle T, r\rangle\| x-\mathbf{M}\left(P_{r}\right)\right] .
\end{aligned}
$$

On account of (see [Fe, 4.3.2(2)])

$$
\int_{5 / 8}^{7 / 8} \int_{\partial \mathbf{C}_{r}}|\mathbf{q} x|^{2} d\|\langle T, r\rangle\| x d r \leq \int_{\mathbf{C}}|\mathbf{q} x|^{2} d\|T\| x
$$

and

$$
\int_{5 / 8}^{7 / 8} \mathbf{M}(\langle T, r\rangle) d r \leq \mathbf{M}\left(T\left\llcorner\left(\mathbf{C}_{7 / 8} \backslash \mathbf{C}_{5 / 8}\right)\right)\right.
$$


hence also (observe $\mathbf{M}\left(P_{r}\right)=r^{n} \mathbf{M}\left(P_{1}\right)$ )

$$
\begin{aligned}
& \int_{5 / 8}^{7 / 8}\left[\mathbf{M}(\langle T, r\rangle)-\frac{n}{r} \mathbf{M}\left(P_{r}\right)\right] d r \\
& \leq \mathbf{M}\left(T\left\llcorner\left(\mathbf{C}_{7 / 8} \backslash \mathbf{C}_{5 / 8}\right)\right)-\mathbf{M}\left(P_{7 / 8}\right)+\mathbf{M}\left(P_{5 / 8}\right) \leq \mathbf{E}(T, 1),\right.
\end{aligned}
$$

we may select $r \in] \frac{5}{8}, \frac{7}{8}[$ such that

$$
\begin{aligned}
& \int_{\partial \mathbf{C}_{r}}|\mathbf{q} x|^{2} d\|\langle T, r\rangle\| x \leq 12 \int_{\mathbf{C}}|\mathbf{q} x|^{2} d\|T\| x, \\
& \mathbf{M}(\langle T, r\rangle) \leq 12 \mathbf{M}(T), \\
& \mathbf{M}(\langle T, r\rangle) \leq \frac{n}{r} \mathbf{M}\left(P_{r}\right)+12 \mathbf{E}(T, 1),
\end{aligned}
$$

and $\langle T, r\rangle$ is rectifiable (cf. [Fe, 4.3.6]). With this choice of $r,(4.3)$ and $\left[1-(1-\vartheta)^{n}\right] \leq n \vartheta$ give

$$
\mathbf{E}\left(\Sigma_{r, \vartheta}, 1\right) \leq 12 \vartheta r \mathbf{E}(T, 1)+\frac{6}{\vartheta r} \int_{\mathbf{C}}|\mathbf{q} x|^{2} d\|T\| x
$$

Similarly, denoting by $\tilde{P}_{\varrho}$ the image of $\left(\mathbf{p}_{\#} T\right)\left\llcorner\left(\mathbf{U} \backslash \mathbf{U}_{\varrho}\right)=\left(\mathbf{E}^{n}\left\llcorner\left(\mathbf{U} \backslash \mathbf{U}_{\varrho}\right)\left\llcorner\iota_{m}\right.\right.\right.\right.$ under the inclusion $\mathbb{R}^{n} \rightarrow \mathbb{R}^{n} \times \mathbb{R}^{k}$ and using the homotopy

$$
\tilde{h}^{\vartheta}(t, x)=x+t(\vartheta \mathbf{p} x,-\mathbf{q} x)=(1-t) x+t(1+\vartheta)(\mathbf{p} x, 0)
$$

we define, similarly as in [Bo, Sec. 5],

$$
\tilde{\Sigma}_{r, \vartheta}=\tilde{P}_{(1+\vartheta) r}+\tilde{h}_{\#}^{\vartheta}(\llbracket 0,1 \rrbracket \times\langle T, r\rangle) .
$$

For $r$ as above and $0<\vartheta \leq \frac{1}{7}$, we then have

$$
\begin{aligned}
& \tilde{\Sigma}_{r, \vartheta}=\tilde{\Sigma}_{r, \vartheta}\left\llcorner\left(\mathbf{C} \backslash \mathbf{C}_{r}\right), \quad \tilde{\Sigma}_{r, \vartheta}\left\llcorner\left(\mathbf{C} \backslash \mathbf{C}_{(1+\vartheta) r}\right)=P_{1}\left\llcorner\left(\mathbf{C} \backslash \mathbf{C}_{(1+\vartheta) r}\right),\right.\right.\right. \\
& \mathbf{p}_{\#}\left(T_{r}+\tilde{\Sigma}_{r, \vartheta}\right)=\mathbf{p}_{\#} P_{1}=\left(\mathbf { E } ^ { n } \llcorner \mathbf { U } ) \left\llcorner\iota_{m}, \quad \partial\left(T_{r}+\tilde{\Sigma}_{r, \vartheta}\right)=\partial P_{1},\right.\right.
\end{aligned}
$$

and obtain the excess estimate $\left(\right.$ noting $\operatorname{Lip} \tilde{h}^{\vartheta}(t, \cdot) \leq 1+t \vartheta$ and $\left[(1+\vartheta)^{n}-1\right] \leq(1+\vartheta)^{n-1} n \vartheta$ this time)

$$
\mathbf{E}\left(\tilde{\Sigma}_{r, \vartheta}, 1\right) \leq(1+\vartheta)^{n-1}\left[12 \vartheta r \mathbf{E}(T, 1)+\frac{6}{\vartheta r} \int_{\mathbf{C}}|\mathbf{q} x|^{2} d\|T\| x\right] .
$$

On account of (4.2) and the hypothesis spt $T_{7 / 8} \subset \mathbf{B}^{n+k}(0, R)$, which by the construction of $\Sigma_{r, \vartheta}$ implies spt $\left(\Sigma_{r, \vartheta}-T_{r}\right) \subset \mathbf{B}^{n+k}(0, R)$, we can apply the $(F, \omega)$ minimality of $T$ to get

$$
\mathbf{F}\left(T_{r}\right) \leq \mathbf{F}\left(\Sigma_{r, \vartheta}\right)+\omega(R) \mathbf{M}\left(\Sigma_{r, \vartheta}\right) .
$$


On the other hand, in view of (4.5) we can make use of the ellipticity of $F_{0}(x, \xi)=F(0, \xi)$ (we also write $F_{0}(\xi)$ for $F(0, \xi)$ in the sequel) to obtain

$$
\begin{aligned}
\lambda 2^{-n} \mathbf{E}\left(T, \frac{1}{2}\right) & =\lambda\left[\mathbf{M}\left(T_{1 / 2}\right)-\mathbf{M}\left(P_{1 / 2}\right)\right] \\
& \leq \lambda\left[\mathbf{M}\left(T_{r}+\tilde{\Sigma}_{r, \vartheta}\right)-\mathbf{M}\left(P_{1}\right)\right] \leq \mathbf{F}_{0}\left(T_{r}+\tilde{\Sigma}_{r, \vartheta}\right)-\mathbf{F}_{0}\left(P_{1}\right) \\
& =\mathbf{F}\left(T_{r}+\tilde{\Sigma}_{r, \vartheta}\left\llcorner\mathbf{C}_{(1+\vartheta) r}\right)+\left(\mathbf{F}_{0}-\mathbf{F}\right)\left(T_{r}+\tilde{\Sigma}_{r, \vartheta}\left\llcorner\mathbf{C}_{(1+\vartheta) r}\right)-\mathbf{F}_{0}\left(P_{(1+\vartheta) r}\right) .\right.\right.
\end{aligned}
$$

Introducing $S_{r, \vartheta}=\Sigma_{r, \vartheta}+\tilde{\Sigma}_{r, \vartheta}\left\llcorner\mathbf{C}_{(1+\vartheta) r}\right.$ and recalling (4.2), (4.5) we deduce from (4.7) that the last expression does not exceed

$$
\begin{aligned}
& \mathbf{F}\left(S_{r, \vartheta}\right)+\omega(R) \mathbf{M}\left(\Sigma_{r, \vartheta}\right)+\left(\mathbf{F}_{0}-\mathbf{F}\right)\left(T_{r}+\tilde{\Sigma}_{r, \vartheta}\left\llcorner\mathbf{C}_{(1+\vartheta) r}\right)-\mathbf{F}_{0}\left(P_{(1+\vartheta) r}\right)\right. \\
& =\mathbf{F}_{0}\left(S_{r, \vartheta}\right)-\mathbf{F}_{0}\left(P_{(1+\vartheta) r}\right)+\left(\mathbf{F}_{0}-\mathbf{F}\right)\left(T_{r}\right)-\left(\mathbf{F}_{0}-\mathbf{F}\right)\left(\Sigma_{r, \vartheta}\right)+\omega(R) \mathbf{M}\left(\Sigma_{r, \vartheta}\right),
\end{aligned}
$$

hence we arrive at

$$
\begin{aligned}
\lambda 2^{-n} \mathbf{E}(T, & \left.\frac{1}{2}\right) \leq \mathbf{F}_{0}\left(S_{r, \vartheta}\right)-\mathbf{F}_{0}\left(P_{(1+\vartheta) r}\right) \\
& +\omega(R) \mathbf{M}\left(\Sigma_{r, \vartheta}\right)+\left(\mathbf{F}_{0}-\mathbf{F}\right)\left(T_{r}\right)-\left(\mathbf{F}_{0}-\mathbf{F}\right)\left(\Sigma_{r, \vartheta}\right) .
\end{aligned}
$$

Now using Taylor expansion of $F_{0}(\xi)$ at $\xi=\overrightarrow{\mathbf{e}}$ for $|\xi-\overrightarrow{\mathbf{e}}|<\sqrt{2}$ and recalling (1.1)-(1.3) we find

$$
\begin{aligned}
\mathbf{F}_{0}\left(S_{r, \vartheta}\right)- & \mathbf{F}_{0}\left(P_{(1+\vartheta) r}\right) \\
=\int_{\mathbf{C}_{(1+\vartheta) r}} & F_{0}\left(\vec{S}_{r, \vartheta}(x)\right) d\left\|S_{r, \vartheta}\right\| x-\int_{\mathbf{C}_{(1+\vartheta) r}} F_{0}(\overrightarrow{\mathbf{e}}) d\left\|P_{(1+\vartheta) r}\right\| \\
\leq \int_{\mathbf{C}_{(1+\vartheta) r}} & {\left[F_{0}(\overrightarrow{\mathbf{e}})+D F_{0}(\overrightarrow{\mathbf{e}})\left(\vec{S}_{r, \vartheta}-\overrightarrow{\mathbf{e}}\right)\right] d\left\|S_{r, \vartheta}\right\| x } \\
& +\Lambda \int_{\mathbf{C}_{(1+\vartheta) r}}\left|\vec{S}_{r, \vartheta}(x)-\overrightarrow{\mathbf{e}}\right|^{2} d\left\|S_{r, \vartheta}\right\| x-\int_{\mathbf{C}_{(1+\vartheta) r}} F_{0}(\overrightarrow{\mathbf{e}}) d\left\|P_{(1+\vartheta) r}\right\|,
\end{aligned}
$$

where $D F_{0}(\overrightarrow{\mathbf{e}}) \overrightarrow{\mathbf{e}}=F_{0}(\overrightarrow{\mathbf{e}})$, by homogeneity, and

$$
\int_{\mathbf{C}_{(1+\vartheta) r}} D F_{0}(\overrightarrow{\mathbf{e}}) \vec{S}_{r, \vartheta}(x) d\left\|S_{r, \vartheta}\right\| x=\int_{\mathbf{C}_{(1+\vartheta) r}} D F_{0}(\overrightarrow{\mathbf{e}}) \overrightarrow{\mathbf{e}} d\left\|P_{(1+\vartheta) r}\right\|,
$$

because $\partial S_{r, \vartheta}=\partial P_{(1+\vartheta) r}$, by (4.2) and (4.5), and the integrals are just the evaluations of $S_{r, \vartheta}$ and $P_{(1+\vartheta) r}$ on the constant (hence exact) differential $n$-form $D F_{0}(\overrightarrow{\mathbf{e}})$. Consequently, by (4.4), (4.6), and (1.24),

$$
\begin{aligned}
\mathbf{F}_{0}\left(S_{r, \vartheta}\right)-\mathbf{F}_{0}\left(P_{(1+\vartheta) r}\right) & \leq \Lambda \int_{\mathbf{C}_{(1+\vartheta) r}}\left|\vec{S}_{r, \vartheta}-\overrightarrow{\mathbf{e}}\right|^{2} d\left\|S_{r, \vartheta}\right\| \\
& \leq \operatorname{const}(n) \Lambda\left[\vartheta \mathbf{E}(T, 1)+\frac{1}{\vartheta} \int_{\mathbf{C}}|\mathbf{q} x|^{2} d\|T\| x\right] .
\end{aligned}
$$

To estimate the other terms appearing in (4.8) we introduce $f(x)=F(0, \overrightarrow{\mathbf{e}})-F(x, \overrightarrow{\mathbf{e}})$ and observe 


$$
\begin{aligned}
& \left|\left(\mathbf{F}_{0}-\mathbf{F}\right)\left(T_{r}\right)-\left(\mathbf{F}_{0}-\mathbf{F}\right)\left(P_{r}\right)\right| \\
& \leq \iint_{0}^{1}\left\|D_{(2)} F(0,(1-t) \overrightarrow{\mathbf{e}}+t \vec{T}(x))-D_{(2)} F(x,(1-t) \overrightarrow{\mathbf{e}}+t \vec{T}(x))\right\||\vec{T}(x)-\overrightarrow{\mathbf{e}}| d t d\left\|T_{r}\right\| x \\
& \quad+\left|\int f(x) d\left\|T_{r}\right\| x-\int f(x) d\left\|P_{r}\right\| x\right| \\
& \leq \int \kappa(|x|)|\vec{T}(x)-\overrightarrow{\mathbf{e}}| d\left\|T_{r}\right\| x+\int|f(x)-f(\mathbf{p} x, 0)| d\left\|T_{r}\right\| x \\
& \quad+\left|\int f(\mathbf{p} x, 0) d\left\|T_{r}\right\| x-\int f(\mathbf{p} x, 0) d\left\|P_{r}\right\| x\right| \\
& \leq \frac{1}{2} \vartheta \int|\vec{T}(x)-\overrightarrow{\mathbf{e}}|^{2} d\left\|T_{r}\right\| x+\frac{1}{2 \vartheta} \kappa(R)^{2} \mathbf{M}\left(T_{r}\right)+\int \mu(|\mathbf{q} x|) d\left\|T_{r}\right\| x \\
& \quad+\kappa(R)\left[\mathbf{M}\left(T_{r}\right)-\mathbf{M}\left(P_{r}\right)\right] \\
& \leq(\vartheta+\kappa(R)) \mathbf{E}(T, 1)+\frac{1}{2 \vartheta} \kappa(R)^{2} \mathbf{M}(T)+\int \mu(|\mathbf{q} x|) d\left\|T_{r}\right\| .
\end{aligned}
$$

Here we have used (1.6), (1.7), (1.5), i.e. $|f(x)| \leq \kappa(|x|) \leq \kappa(R)$, and the inequality

$$
\left|\int \varphi(\mathbf{p} x) d\left\|T_{r}\right\| x-\int \varphi(\mathbf{p} x) d\left\|P_{r}\right\| x\right| \leq \sup |\varphi| \mathbf{E}(T, 1)
$$

for continuous functions $\varphi$ on $\mathbb{R}^{n}$, an inequality which one readily proves by approximating $\varphi$ with finitely valued functions. Recalling the definition (1.9) of $\widehat{\mu}$ we use the inequalities $\mu(s)-s^{2} \leq \widehat{\mu}\left(\frac{1}{2}\right)$ for $0 \leq s \leq \frac{1}{2}$ and $\mu(s) \leq 2 s \mu\left(\frac{1}{2}\right) \leq s^{2}+\mu\left(\frac{1}{2}\right)^{2} \leq s^{2}+\kappa\left(\frac{1}{2}\right)^{2}$ for $s>\frac{1}{2}$ to estimate

$$
\int \mu(|\mathbf{q} x|) d\left\|T_{r}\right\| x \leq\left(\kappa(R)^{2}+\widehat{\mu}(R)\right) \mathbf{M}(T)+\int_{\mathbf{C}}|\mathbf{q} x|^{2} d\|T\| x .
$$

Collecting the estimates we have proved

$$
\begin{aligned}
\mid\left(\mathbf{F}_{0}-\mathbf{F}\right) & \left(T_{r}\right)-\left(\mathbf{F}_{0}-\mathbf{F}\right)\left(P_{r}\right) \mid \\
& \leq 2 \vartheta \mathbf{E}(T, 1)+\left[\frac{2}{\vartheta} \kappa(R)^{2}+\widehat{\mu}(R)\right] \mathbf{M}(T)+\int_{\mathbf{C}}|\mathbf{q} x|^{2} d\|T\| x .
\end{aligned}
$$

Similarly as above we estimate

$$
\begin{aligned}
& \left|\left(\mathbf{F}_{0}-\mathbf{F}\right)\left(\Sigma_{r, \vartheta}\right)-\left(\mathbf{F}_{0}-\mathbf{F}\right)\left(P_{r}\right)\right| \\
& \quad \leq(1+\kappa(R)) \mathbf{E}\left(\Sigma_{r, \vartheta}, 1\right)+\frac{1}{2} \kappa(R)^{2} \mathbf{M}\left(\Sigma_{r, \vartheta}\right)+\int_{\mathbf{C}} \mu(|\mathbf{q} x|) d\left\|\Sigma_{r, \vartheta}\right\| x .
\end{aligned}
$$

Here we apply (4.4) to estimate $\mathbf{E}\left(\Sigma_{r, \vartheta}, 1\right)$, and the inequality

$$
\mathbf{M}\left(\Sigma_{r, \vartheta}\right)=\mathbf{E}\left(\Sigma_{r, \vartheta}, 1\right)+\mathbf{M}\left(P_{r}\right) \leq \mathbf{E}\left(\Sigma_{r, \vartheta}, 1\right)+\mathbf{M}(T)
$$

to estimate $\mathbf{M}\left(\Sigma_{r, \vartheta}\right)$. We use again $\mu(s) \leq \widehat{\mu}\left(\frac{1}{2}\right)+s^{2}$ for $0<s \leq \frac{1}{2}$ and $\mu(s) \leq 2 s \mu\left(\frac{1}{2}\right)$ for $s>\frac{1}{2}$ to get

$$
\int_{\mathbf{C}} \mu(|\mathbf{q} x|) d\left\|\Sigma_{r, \vartheta}\right\| x \leq \widehat{\mu}\left(\frac{1}{2}\right) \mathbf{M}\left(\Sigma_{r, \vartheta}\right)+\int_{|\mathbf{q} x| \leq \frac{1}{2}}|\mathbf{q} x|^{2} d\left\|\Sigma_{r, \vartheta}\right\| x+2 \mu\left(\frac{1}{2}\right) \int_{|\mathbf{q} x|>\frac{1}{2}}|\mathbf{q} x| d\left\|\Sigma_{r, \vartheta}\right\| x .
$$


Noting

$$
\left|\mathbf{q}\left(h^{\vartheta}\left(t, x^{\prime}\right)\right)\right|^{2}=(1-t)^{2}\left|\mathbf{q} x^{\prime}\right|^{2} \leq \min \left(\left|\mathbf{q} x^{\prime}\right|,\left|\mathbf{q} x^{\prime}\right|^{2}\right)
$$

if $\left|\mathbf{q}\left(h^{\vartheta}\left(t, x^{\prime}\right)\right)\right| \leq \frac{1}{2}$ and $x^{\prime} \in \operatorname{spt}\langle T, r\rangle$, we estimate

$$
\begin{aligned}
& \int_{|\mathbf{q} x| \leq \frac{1}{2}}|\mathbf{q} x|^{2} d\left\|\Sigma_{r, \vartheta}\right\| x \\
& \leq \int_{0}^{1} \int_{\partial \mathbf{C}_{r}} \min \left(\left|\mathbf{q} x^{\prime}\right|,\left|\mathbf{q} x^{\prime}\right|^{2}\right)\left|\left(\vartheta \mathbf{p} x^{\prime}, \mathbf{q} x^{\prime}\right)\right|(1-\vartheta t)^{n-1} d\|\langle T, r\rangle\| x^{\prime} d t \\
& \leq(1+\vartheta r) \int_{\partial \mathbf{C}_{r}}\left|\mathbf{q} x^{\prime}\right|^{2} d\|\langle T, r\rangle\| x^{\prime} \leq 12(1+\vartheta r) \int_{\mathbf{C}}|\mathbf{q} x|^{2} d\|T\| x .
\end{aligned}
$$

Furthermore,

$$
\begin{aligned}
& 2 \mu\left(\frac{1}{2}\right) \int_{|\mathbf{q} x|>\frac{1}{2}}|\mathbf{q} x| d\left\|\Sigma_{r, \vartheta}\right\| x \\
& \leq 2 \mu\left(\frac{1}{2}\right) \int_{0}^{1} \int_{\partial \mathbf{C}_{r}}(1-t)\left|\mathbf{q} x^{\prime}\left\|\left(\vartheta \mathbf{p} x^{\prime}, \mathbf{q} x^{\prime}\right) \mid(1-\vartheta t)^{n-1} d\right\|\langle T, r\rangle \| x^{\prime} d t\right. \\
& \leq \mu\left(\frac{1}{2}\right)^{2} \vartheta^{2} r^{2} \mathbf{M}(\langle T, r\rangle)+\left[1+2 \mu\left(\frac{1}{2}\right)\right] \int_{\partial \mathbf{C}_{r}}\left|\mathbf{q} x^{\prime}\right|^{2} d\|\langle T, r\rangle\| x^{\prime} \\
& \leq \mu\left(\frac{1}{2}\right)^{2} \mathbf{M}(T)+24\left[1+\mu\left(\frac{1}{2}\right)\right] \int_{\mathbf{C}}|\mathbf{q} x|^{2} d\|T\| x .
\end{aligned}
$$

Collecting terms and using the monotonicity of $\kappa, \mu, \widehat{\mu}$ as well as $\mu \leq \kappa$ we arrive at

$$
\begin{aligned}
\left|\left(\mathbf{F}_{0}-\mathbf{F}\right)\left(\Sigma_{r, \vartheta}\right)-\left(\mathbf{F}_{0}-\mathbf{F}\right)\left(P_{r}\right)\right| & \leq 24 \vartheta \mathbf{E}(T, 1)+5\left[\kappa(R)^{2}+\widehat{\mu}(R)\right] \mathbf{M}(T) \\
+ & \frac{80}{\vartheta}\left(1+\kappa(R)^{2}+\widehat{\mu}(R)\right) \int_{\mathbf{C}}|\mathbf{q} x|^{2} d\|T\| x .
\end{aligned}
$$

Combining (4.8)-(4.11) and estimating the term $\omega(R) \mathbf{M}\left(\Sigma_{r, \vartheta}\right)$ similarly as above we finally conclude

$$
\begin{aligned}
\lambda 2^{-n} \mathbf{E}\left(T, \frac{1}{2}\right) \leq & \operatorname{const}(n) \Lambda \vartheta \mathbf{E}(T, 1)+\frac{7}{\vartheta}\left(\kappa^{2}(R)+\widehat{\mu}(R)+\omega(R)\right) \mathbf{M}(T) \\
& +\operatorname{const}(n) \frac{\Lambda}{\vartheta}\left(1+\kappa^{2}(R)+\widehat{\mu}(R)+\omega(R)\right) \int_{\mathbf{C}}|\mathbf{q} x|^{2} d\|T\| .
\end{aligned}
$$

This is true for $0<\vartheta \leq \frac{1}{7}$, but increasing the constants if necessary we obtain the same inequality trivially for all $\vartheta>0$, and (4.1) follows.

4.2 Lemma (reverse Poincaré inequality). Suppose $T \in \mathcal{T}_{*}(n, k, m)$ is $(F, \omega)$ minimizing in $\mathbf{C}$ with support in $\mathbf{B}^{n} \times \mathbf{B}^{k}(0, h), h>0$, and $r=\sqrt{1+h^{2}}$. Then the inequality

$$
\mathbf{E}\left(T, \frac{1}{5}\right) \leq C_{12}\left[\chi(r) \mathbf{M}(T)+(1+\chi(r)) \int_{\mathbf{C}}|\mathbf{q} x|^{2} d\|T\| x\right]
$$

holds with $C_{12}=\operatorname{const}(n, \lambda, \Lambda)$ and $\chi(r)=\kappa(r)^{2}+\widehat{\mu}(r)+\omega(r)$. 
The point here is that $\chi(r)$ is small in later applications so that (4.12) is indeed a reverse Poincaré inequality, up to a small extra term. Multiplicity $m=0$ is admitted in Lemma 4.2 but irrelevant for later applications.

Again we point out that the condition $0 \in \operatorname{spt} T$ from the definition of $\mathcal{T}_{i}(n, k, m)$ is not needed in the proof.

Proof. We consider the boundary situation $T \in \mathcal{T}_{b}(n, k, m)$ and $n$-balls $\mathbf{B}_{\sigma}(y) \subset \mathbf{U}$ such that $y \in \mathbf{U}^{n-1} \times\{0\}$ or $\mathbf{B}_{\sigma}(y) \cap \mathbf{U}^{n-1} \times\{0\}=\emptyset$. We want to apply Lemma 4.1 (with the comment following it) to the scaled currents $T_{\sigma}=\left(\boldsymbol{\mu}_{\sigma \#}^{-1} \boldsymbol{\tau}_{(y, 0) \#}^{-1} T\right)\llcorner\mathbf{C}$. (Note that we may have $T_{\sigma} \in \mathcal{T}_{i}(n, k, 0)$ in the case $T \in \mathcal{T}_{b}(n, k, 1)$; this is the reason for admitting $m=0$ in Lemma 4.1.) Clearly, $T_{\sigma}$ satisfies the hypothesis of Lemma 4.1 with respect to the integrand $F_{\sigma}(x, \xi)=F((y, 0)+\sigma x, \xi)$ and the function $\omega_{\sigma}(\varrho)=\omega(\sigma \varrho)$. For the structure constants and moduli of $F_{\sigma}$ we have $\lambda_{\sigma}=\lambda, \Lambda_{\sigma}=\Lambda, \kappa_{\sigma}(\varrho)=\kappa(\sigma \varrho), \mu_{\sigma}(\varrho)=\mu(\sigma \varrho), \widehat{\mu_{\sigma}}(\varrho) \leq \widehat{\mu}(\sigma \varrho)$, and $T_{\sigma}$ has support in $\mathbf{B}^{n+k}\left(0, \sigma^{-1} r\right)$. Therefore, (4.1) implies

$$
\begin{aligned}
\mathbf{E}\left(T, y, \frac{1}{2} \sigma\right) \leq & \vartheta \mathbf{E}(T, y, \sigma) \\
& +\frac{1}{\vartheta}\left[c \sigma^{-n-2} \int_{\mathbf{C}_{\sigma}(y)}|\mathbf{q} x|^{2} d\|T\| x+c^{\prime} \sigma^{-n} \mathbf{M}\left(T\left\llcorner\mathbf{C}_{\sigma}(y)\right)\right],\right.
\end{aligned}
$$

where we have abbreviated $c=C_{11}(1+\chi(r))$ and $c^{\prime}=C_{11} \chi(r)$. For balls $\mathbf{B}_{\varrho}(y) \subset \mathbf{U}$ which intersect $\mathbf{U}^{n-1} \times\{0\}$ we define $y^{\prime}=\left(y_{1}, \ldots, y_{n-1}, 0\right)$ and observe

$$
\mathbf{B}_{\varrho}(y) \subset \mathbf{B}_{2 \varrho}\left(y^{\prime}\right) \subset \mathbf{B}_{4 \varrho}\left(y^{\prime}\right) \subset \mathbf{B}_{5 \varrho}(y),
$$

hence (4.13) applied to $\mathbf{B}_{2 \varrho}\left(y^{\prime}\right)$ gives, provided $\mathbf{B}_{5 \varrho}(y) \subset \mathbf{U}$,

$$
\begin{aligned}
& 2^{-n} \mathbf{E}(T, y, \varrho) \leq \mathbf{E}\left(T, y^{\prime}, 2 \varrho\right) \\
& \leq \vartheta\left(\frac{4}{5}\right)^{n} \mathbf{E}(T, y, 5 \varrho)+\frac{1}{\vartheta}\left[c(4 \varrho)^{-n-2} \int_{\mathbf{C}_{5 \varrho}(y)}|\mathbf{q} x|^{2} d\|T\| x+c^{\prime}(4 \varrho)^{-n} \mathbf{M}\left(T\left\llcorner\mathbf{C}_{5 \varrho}(y)\right)\right] .\right.
\end{aligned}
$$

Increasing $c$ and $c^{\prime}$ by a factor const $(n)$ we therefore have

$$
\mathbf{E}\left(T, y, \frac{1}{5} \sigma\right) \leq \vartheta \mathbf{E}(T, y, \sigma)+\frac{1}{\vartheta}\left[c \sigma^{-n-2} \int_{\mathbf{C}}|\mathbf{q} x|^{2} d\|T\| x+c^{\prime} \sigma^{-n} \mathbf{M}(T)\right]
$$

for all balls $\mathbf{B}_{\sigma}(y) \subset \mathbf{U}$ and parameters $\vartheta>0$.

With (4.14) we are in a position to apply a covering argument as in [Si2, §2.8]. For this we set

$$
\varphi\left(\mathbf{B}_{\sigma}(y)\right)=\sigma^{n} \mathbf{E}(T, y, \sigma)=\frac{1}{2} \int_{\mathbf{C}_{\sigma}(y)}|\vec{T}(x)-\overrightarrow{\mathbf{e}}|^{2} d\|T\| x
$$

and rewrite (4.14), using also $\sigma^{2}<1$,

$$
\sigma^{2} \varphi\left(\mathbf{B}_{\sigma / 5}(y)\right) \leq \vartheta \sigma^{2} \varphi\left(\mathbf{B}_{\sigma}(y)\right)+\gamma(\vartheta)
$$

with

$$
\gamma(\vartheta)=\frac{1}{\vartheta}\left[c 5^{-n} \int_{\mathbf{C}}|\mathbf{q} x|^{2} d\|T\| x+5^{-n} c^{\prime} \mathbf{M}(T)\right]
$$


We define

$$
Q=\sup \left\{\varrho^{2} \varphi\left(\mathbf{B}_{\varrho}(y)\right): \mathbf{B}_{5 \varrho}(y) \subset \mathbf{U}\right\}
$$

and note $Q \leq 5^{-2} \mathbf{E}(T, 0,1)<\infty$. Covering $\mathbf{B}_{\varrho}(y) \subset \mathbf{U}_{1-\varrho}$ by balls $\mathbf{B}_{\varrho / 25}\left(y_{i}\right)$ with $y_{i} \in$ $\mathbf{B}_{\varrho}(y), i=1, \ldots, M$, and $M=\operatorname{const}(n)$, we deduce from the subadditivity of the functional $\varphi(\cdot)$ on balls in $\mathbf{U}$ and from (4.15)

$$
\begin{aligned}
\varrho^{2} \varphi\left(\mathbf{B}_{\varrho}(y)\right) & \leq \varrho^{2} \sum_{i=1}^{M} \varphi\left(\mathbf{B}_{\varrho / 25}\left(y_{i}\right)\right) \\
& \leq 5^{2} \sum_{i=1}^{M}\left[\vartheta\left(\frac{\varrho}{5}\right)^{2} \varphi\left(\mathbf{B}_{\varrho / 5}\left(y_{i}\right)\right)+\gamma(\vartheta)\right] \\
& \leq 5^{2} \vartheta Q M+5^{2} \gamma(\vartheta) M .
\end{aligned}
$$

Taking the supremum over all balls $\mathbf{B}_{\varrho}(y)$ with $\mathbf{B}_{5 \varrho}(y) \subset \mathbf{U}$ in this inequality we get

$$
Q \leq 5^{2} \vartheta Q M+5^{2} M \gamma(\vartheta)
$$

Fixing now $\vartheta=(50 M)^{-1}$ we see $Q \leq 50 M \gamma(\vartheta)$ from (4.19), and from (4.17) we obtain

$$
\varrho^{2} \varphi\left(\mathbf{B}_{\varrho}(y)\right) \leq 50 M \gamma(\vartheta)
$$

i.e.

$$
\mathbf{E}(T, y, \varrho) \leq \varrho^{-n-2} 50 M \gamma(\vartheta)
$$

Applying this with $y=0, \varrho=\frac{1}{5}$ and recalling (4.16) we have proved (4.12).

The proof in the interior situation $T \in \mathcal{T}_{i}(n, k, m)$ is simpler, as one can use directly (4.13) for all balls $\mathbf{B}_{\sigma}(y) \subset \mathbf{U}$.

\section{Excess decay}

As is well known [Alm1], [Alm2], [Bo], [Fe, §5.3], [Ha1] the crucial step in the regularity proof is excess improvement, an idea introduced by Almgren. If $T$ is $(F, \omega)$ minimizing with small excess $\mathbf{E}(T, 1)$ in the cylinder of radius 1 over the $n$-plane $\mathbb{R}^{n} \times\{0\}^{k}$ in $\mathbb{R}^{n+k}$, then one shows that the excess of $T$ over some $n$-plane $S$ close to $\mathbb{R}^{n} \times\{0\}^{k}$ and in a cylinder with smaller radius is substantially smaller than $\mathbf{E}(T, 1)$. By iteration of this argument one can prove that the excess of $T$ in concentric cylinders of radius $r$ over some $n$-plane decreases to zero with $r$, and from this excess decay one deduces finally the regularity of $T$ in a small cylinder.

In this section we first prove (Lemma 5.1) that the square integral of the height of $T$ over a tilted $n$-plane $S$, which is close to $\mathbb{R}^{n} \times\{0\}^{k}$, can be dominated by a small constant times $\mathbf{E}(T, 1)+\kappa\left(\frac{1}{2}\right)^{2}+\widehat{\mu}\left(\frac{1}{2}\right)+\omega\left(\frac{1}{2}\right)$, provided this quantity is small. This $n$-plane $S$ is found as the graph of the differential at zero of the $A$-harmonic function $u$ which is associated by Lemma 3.3 with the approximatively $A$-harmonic Lipschitz approximation $g$ of $T$ (Lemma 3.1 and Lemma 3.2). Then we use the reverse Poincaré inequality (Lemma 4.2) to replace the square integral of the height of $T$ over the tilted plane $S$ by the excess of $T$ over this plane 
in a cylinder of smaller radius $\vartheta \in] 0,1[$. The result is Lemma 5.2, an estimate of this latter excess by $\vartheta \mathbf{E}(T, 1)$ plus extra terms involving only the (small) quantity $\kappa\left(\frac{1}{2}\right)^{2}+\widehat{\mu}\left(\frac{1}{2}\right)+\omega\left(\frac{1}{2}\right)$. The iteration of Lemma 5.2 then leads to the desired excess decay in Lemma 5.4.

We assume in this section that $F$ is an elliptic integrand with ellipticity constant $\lambda$, bound $\Lambda$, and moduli $\kappa, \mu, \widehat{\mu}, \nu$ as in Section 1. In Lemma 5.4 we need the extra assumption (1.15) on $\kappa, \widehat{\mu}$ and the function $\omega$ which appears in the $(F, \omega)$ minimality condition for the rectifiable $n$-current $T$.

5.1 Lemma (improving the $\boldsymbol{L}^{2}$ height estimate by tilting). Suppose $T \in \mathcal{T}_{b}(n, k, 1)$ or $T \in \mathcal{T}_{i}(n, k, m)$ with (2.12), $T$ is $(F, \omega)$ minimizing in $\mathbf{C}, \chi=\kappa\left(\frac{1}{2}\right)^{2}+\widehat{\mu}\left(\frac{1}{2}\right)+\omega\left(\frac{1}{2}\right) \leq 1$, $E=\mathbf{E}(T, 1), 0<\epsilon \leq 1,0<\sigma \leq 1,0<\gamma \leq 1$ and a smallness condition

$$
C_{17}\left[\gamma^{-2} \sqrt{E}+\sqrt{\sigma}+\nu(\gamma+\sqrt{\chi})\right] \leq \delta(n, k, \lambda, \Lambda, \epsilon)
$$

is satisfied, where $\delta(n, k, \lambda, \Lambda, \epsilon) \in] 0,1]$ is from Lemma 3.3 and $C_{17}=\operatorname{const}(n, m, \lambda, \Lambda)$. Then there exist $L \in \operatorname{Hom}\left(\mathbb{R}^{n}, \mathbb{R}^{k}\right)$ and $v \in \mathbb{R}^{n+k}$ perpendicular to the $n$-plane $S=\operatorname{graph}(L)$ such that for $0<\eta \leq \frac{1}{17}$ the inequality

$$
\int_{\mathbf{C}_{\eta / 2}} \operatorname{dist}(x-v, S)^{2} d\|T\| x \leq C_{18}\left(\gamma^{-2} E^{1 / n}+\eta^{n+4}+\epsilon\right)\left(E+\sigma^{-1} \chi\right)
$$

is valid with a constant $C_{18}=\operatorname{const}(n, m, \lambda, \Lambda)$. Moreover

$$
\|L\| \leq C_{18} \sqrt{E+\sigma^{-1} \chi}
$$

holds, and in the case $T \in \mathcal{T}_{b}(n, k, 1)$ we have $L=0$ on $\mathbb{R}^{n-1} \times\{0\}, v=0$, while in the case $T \in \mathcal{T}_{i}(n, k, m)$

$$
|v| \leq C_{18}\left(\sqrt{E+\sigma^{-1} \chi}+E^{1 / 2 n}\right) .
$$

Proof. We consider the boundary situation $T \in \mathcal{T}_{b}(n, k, 1)$ and indicate only some modifications of the proof for the case $T \in \mathcal{T}_{i}(n, k, m)$ satisfying (2.12). We fix $A=D^{2} F_{0}^{\S}$, choose $\varrho=\frac{1}{17}$ in Lemma 3.2, and let $g$ be the Lipschitz approximation for $T$ corresponding to $\beta=1$. Then we know from Lemma 3.1, (ii) and (iii)

$$
\int_{\mathbf{U}_{+} \cap \mathbf{U}_{\varrho}}|D g|^{2} d \mathcal{L}^{n} \leq C_{13} E,
$$

where $C_{13}=\operatorname{const}(n, m) \Lambda^{2 n-2}$ in view of our assumption $\omega\left(\frac{1}{2}\right) \leq 1$. Scaling

$$
f=\varrho^{n / 2-1} C_{13}^{-1 / 2}\left(E+\sigma^{-1} \chi\right)^{-1 / 2} g
$$

with a parameter $0<\sigma \leq 1$, we have

$$
\varrho^{2-n} \int_{\mathbf{U}_{+} \cap \mathbf{U}_{\varrho}}|D f|^{2} d \mathcal{L}^{n} \leq 1
$$


and obtain from Lemma 3.2

$$
\int_{\mathbf{U}_{\varrho}} A(D f, D \zeta) d \mathcal{L}^{n} \leq \varrho^{n / 2-1} C_{14}\left[\gamma^{-2} \sqrt{E}+\sqrt{\sigma}+\nu(\gamma+\sqrt{\chi})\right] \sup |D \zeta|
$$

for all functions $\zeta \in \mathbb{C}^{1}\left(\mathbf{U}, \mathbb{R}^{k}\right)$ with compact support in $\mathbf{U}_{+} \cap \mathbf{U}_{\varrho}$, where $0<\gamma \leq 1$ can be chosen arbitrarily and $C_{14}=\max \left\{2 C_{13}^{-1 / 2} C_{10}, 1\right\}$. The hypothesis $\mathbf{C}_{\varrho} \cap \operatorname{spt} T \subset \mathbf{U}_{\varrho}^{n} \times \mathbf{B}_{1 / 3}^{k}$ in Lemma 3.2 is satisfied, by Lemma 2.2, if we choose the constant in (5.1) large enough.

We now apply Lemma 3.3 to obtain, for given $\epsilon>0$, an $A$-harmonic function $u \in$ $W_{(0)}^{1,2}\left(\mathbf{U}_{+} \cap \mathbf{U}_{\varrho}, \mathbb{R}^{k}\right)$ such that

$$
\varrho^{-2} \int_{\mathbf{U}_{+} \cap \mathbf{U}_{\varrho}}|u-g|^{2} d \mathcal{L}^{n} \leq \epsilon C_{13}\left[E+\sigma^{-1} \chi\right]
$$

and

$$
\int_{\mathbf{U}_{+} \cap \mathbf{U}_{\varrho}}|D u|^{2} d \mathcal{L}^{n} \leq C_{13}\left[E+\sigma^{-1} \chi\right]
$$

provided

$$
\varrho^{-n / 2} C_{14}\left[\gamma^{-2} \sqrt{E}+\sqrt{\sigma}+\nu(\gamma+\sqrt{\chi})\right] \leq \delta(n, k, \lambda, \Lambda, \epsilon) .
$$

Noting that $u$ is smooth on $\overline{\mathbf{U}}_{+} \cap \mathbf{U}_{\varrho}$ with $u=0$ on $\mathbf{U}_{\varrho}^{n-1} \times\{0\}$ we define $L=D u(0)$ and observe $L y=y_{n} \ell$ for some $\ell \in \mathbb{R}^{k}$ and all $y \in \mathbb{R}^{n}$. From the a-priori-estimate for solutions to homogeneous constant coefficient elliptic systems

$$
\varrho^{2} \sup _{\mathbf{U}_{+} \cap \mathbf{U}_{\varrho / 2}}\|D u\|^{2}+\varrho^{4} \sup _{\mathbf{U}_{+} \cap \mathbf{U}_{\varrho / 2}}\left\|D^{2} u\right\|^{2} \leq \operatorname{const}(n, \lambda, \Lambda) \varrho^{2-n} \int_{\mathbf{U}_{+} \cap \mathbf{U}_{\varrho}}|D u|^{2} d \mathcal{L}^{n}
$$

(which follows from Caccioppoli's inequality for $u$ and its derivatives of any order, Sobolev's inequality, and Poincaré's inequality, see [Ca], [Gi], [Mo]) we deduce by Taylor expansion

$$
\sup _{y \in \mathbf{U}_{+} \cap \mathbf{U}_{\eta / 2}}|u(y)-L y|^{2} \leq C_{15}\left(\frac{\eta}{\varrho}\right)^{4} \varrho^{2-n} \int_{\mathbf{U}_{+} \cap \mathbf{U}_{\varrho}}|D u|^{2} d \mathcal{L}^{n}
$$

for $0<\eta \leq \varrho$, where $C_{15}=\operatorname{const}(n, \lambda, \Lambda)$. (In the interior situation $T \in \mathcal{T}_{i}(n, k, m)$ we have the same estimate for $|u(y)-u(0)-L y|^{2}$ on $\mathbf{U}_{\eta / 2}$.) Combining (5.9) with (5.5) and (5.6) we get

$$
\begin{aligned}
\int_{\mathbf{U}_{+} \cap \mathbf{U}_{\eta / 2}} & |g(y)-L y|^{2} d \mathcal{L}^{n} y \\
& \leq 2 \int_{\mathbf{U}_{+} \cap \mathbf{U}_{\eta / 2}}|g(y)-u(y)|^{2} d \mathcal{L}^{n} y+2 \int_{\mathbf{U}_{+} \cap \mathbf{U}_{\eta / 2}}|u(y)-L y|^{2} d \mathcal{L}^{n} y \\
& \leq C_{16}\left(\epsilon+\eta^{n+4}\right)\left(E+\sigma^{-1} \chi\right)
\end{aligned}
$$

with $C_{16}=\operatorname{const}(n, m, \lambda, \Lambda)$. (In the interior situation we get the same estimate for the integral of $|g(y)-u(0)-L y|^{2}$ on $\left.\mathbf{U}_{\eta / 2} \cdot\right)$ 
Next we estimate with (5.8) and $L=D u(0)$

$$
\sup _{y \in \mathbf{U}_{+} \cap \mathbf{U}_{\varrho / 2}}\|D u(y)-L\|^{2} \leq \operatorname{const}(n, \lambda, \Lambda) \varrho^{-n} \int_{\mathbf{U}_{+} \cap \mathbf{U}_{\varrho}}|D u|^{2} d \mathcal{L}^{n}
$$

and obtain, using (5.6) again,

$$
\begin{aligned}
\|L\| & \leq f_{\mathbf{U}_{+} \cap \mathbf{U}_{\varrho / 2}}\|L-D u(y)\| d \mathcal{L}^{n} y+f_{\mathbf{U}_{+} \cap \mathbf{U}_{\varrho / 2}}\|D u(y)\| d \mathcal{L}^{n} y \\
& \leq \operatorname{const}(n, m, \lambda, \Lambda) \sqrt{E+\sigma^{-1} \chi} .
\end{aligned}
$$

In order to estimate the integral of $\operatorname{dist}(x, S)^{2}$ for the $n$-plane $S=\operatorname{graph}(L)$ we observe

$$
\begin{aligned}
& \int_{\mathbf{C}_{\eta / 2}} \operatorname{dist}(x, S)^{2} d\|T\| x \\
& =\int_{\mathbf{C}_{+} \cap \mathbf{C}_{\eta / 2} \backslash\left(A_{g, \gamma} \times \mathbb{R}^{k}\right)} \ldots d\left\|T_{g}\right\|+\int_{\mathbf{C}_{\eta / 2}} \ldots d\left\|T-T_{g}\right\|+\int_{\mathbf{C}_{\eta / 2} \cap\left(A_{g, \gamma} \times \mathbb{R}^{k}\right)} \ldots d\left\|T_{g}\right\| \\
& =\mathrm{I}+\mathrm{II}+\mathrm{III},
\end{aligned}
$$

where $A_{g, \gamma}$ is the bad set of $g$ corresponding to the parameter $\gamma$ as in Lemma 3.1, (iv). For $x \in \mathbf{C}_{\eta / 2} \cap \operatorname{spt} T$ we have

$$
\begin{aligned}
\operatorname{dist}(x, S) & \leq|\mathbf{q} x-L \mathbf{p} x| \leq|\mathbf{q} x|+|L \mathbf{p} x| \\
& \leq \operatorname{const}(n, m, \lambda, \Lambda)\left[E^{1 / 2 n}+\eta \sqrt{E+\sigma^{-1} \chi}\right]
\end{aligned}
$$

by (5.11) and Lemma 2.2. (We have already noted that (5.7) implies the smallness condition (2.4) necessary to apply Lemma 2.2.) From Lemma 3.1, (i) and (iv) (with $\beta=1$ ) we therefore obtain

$$
|\mathrm{II}|+|\mathrm{III}| \leq \operatorname{const}(n, m, \lambda, \Lambda)\left[E^{1 / n}+\eta^{2}\left(E+\sigma^{-1} \chi\right)\right] \gamma^{-2} E .
$$

Letting $G(y)=(y, g(y))$ we also see from (5.10), taking $\operatorname{dist}(G(y), S) \leq|g(y)-L y|$ and $\operatorname{Lip} g \leq \beta=1$ into account,

$$
\begin{aligned}
|\mathrm{I}| & \leq \int_{\mathbf{U}_{+} \cap \mathbf{U}_{\eta / 2}}|g(y)-L y|^{2} J G(y) d \mathcal{L}^{n} y \\
& \leq \operatorname{const}(n, m, \lambda, \Lambda)\left(\epsilon+\eta^{n+4}\right)\left(E+\sigma^{-1} \chi\right) .
\end{aligned}
$$

The result is

$$
\int_{\mathbf{C}_{\eta / 2}} \operatorname{dist}(x, S)^{2} d\|T\| x \leq \operatorname{const}(n, m, \lambda, \Lambda)\left(\gamma^{-2} E^{1 / n}+\eta^{n+4}+\epsilon\right)\left(E+\sigma^{-1} \chi\right) .
$$

With this inequality and (5.11) we have proved all the assertions of the Lemma in the boundary case under the smallness condition (5.7), i.e. (5.1). In the interior case we obtain the same result and note additionally

$$
\begin{aligned}
\frac{1}{3} \int_{\mathbf{U}_{\varrho / 2}}|u(0)+L y|^{2} d \mathcal{L}^{n} y & \leq \int_{\mathbf{U}_{\varrho / 2}}|u(0)+L y-g(y)|^{2} d \mathcal{L}^{n} y+\int_{\mathbf{U}_{\varrho / 2}}|g|^{2} d \mathcal{L}^{n} \\
& \leq \operatorname{const}(n, m, \lambda, \Lambda)\left[(1+\epsilon)\left(E+\sigma^{-1} \chi\right)+E^{1 / n}\right]
\end{aligned}
$$


by the analogue of (5.10), by (5.5), and by Lemma 3.1, (vi). In view of

$$
|u(0)|=\left|f_{\mathbf{U}_{\varrho / 2}}(u(0)+L y) d \mathcal{L}^{n}\right|
$$

this implies the estimate (5.4) for $v$ which equals the orthogonal projection of $u(0)$ onto $S^{\perp}$.

We note that in the boundary case $T \in \mathcal{T}_{b}(n, k, m)$ with multiplicity $m \geq 2$ and with the additional density condition (2.13) the proof above does not work, because we could only prove in Lemma 3.2 that $g$ is approximatively $A$-harmonic on $\mathbf{U}_{+} \cap \mathbf{U}_{\varrho}$ and on $\mathbf{U}_{-} \cap \mathbf{U}_{\varrho}$ separately. Consequently we can find $A$-harmonic functions $u_{+}$near $g$ on $\mathbf{U}_{+} \cap \mathbf{U}_{\varrho}$ and $u_{-}$ on $\mathbf{U}_{-} \cap \mathbf{U}_{\varrho}$ but we do not know that the differentials $D u_{+}(0), D u_{-}(0)$ coincide and, hence, cannot find, with the method above, a tilted $n$-plane $S$ such that (5.2) holds.

In the following Lemma we use, in the interior situation, the modulus $\widehat{\kappa}$ associated with $\kappa$ by (1.9), and we recall $\frac{1}{4} \kappa\left(\frac{1}{2}\right)^{2} \leq \widehat{\kappa}\left(\frac{1}{2}\right)$ if $\kappa\left(\frac{1}{2}\right) \leq 1$.

5.2 Lemma (excess improvement by tilting). For $0<\vartheta \leq \frac{1}{340}$ there exists $a$ positive constant $\delta_{1}(n, k, m, \lambda, \Lambda, \nu(\cdot), \vartheta) \leq 1$ such that the following is true: Whenever $T \in \mathcal{T}_{b}(n, k, 1)$ or $T \in \mathcal{T}_{i}(n, k, m)$ with (2.12) is $(F, \omega)$ minimizing in $\mathbf{C}$ with spt $T \subset \mathbb{R}^{n} \times \mathbf{B}^{k}$ and the smallness conditions

$$
E=\mathbf{E}(T, 1) \leq \delta_{1}, \quad \chi \leq \delta_{1},
$$

are satisfied, where $\chi=\widehat{\kappa}\left(\frac{1}{2}\right)+\omega\left(\frac{1}{2}\right)$ in the interior case $T \in \mathcal{T}_{i}(n, k, m)$ and $\chi=\kappa\left(\frac{1}{2}\right)^{2}+$ $\widehat{\mu}\left(\frac{1}{2}\right)+\omega\left(\frac{1}{2}\right)$ in the boundary case $T \in \mathcal{T}_{b}(n, k, m)$, then there exists a linear isometry, of $\mathbb{R}^{n+k}$ with

$$
\|,-\mathrm{id}\| \leq C_{21} \sqrt{E+\chi}
$$

and with,$=\mathrm{id}$ on $\left(\mathbb{R}^{n-1} \times\{0\}\right) \times\{0\}^{k}$ in the case $T \in \mathcal{T}_{b}(n, k, 1)$, such that

$$
\mathbf{E}\left(,{ }_{\#} T, \vartheta\right) \leq C_{22} \vartheta^{2} \mathbf{E}(T, 1)+C_{21} \chi
$$

Here $C_{22}=\operatorname{const}(n, m, \lambda, \Lambda)$, and $C_{21}=\operatorname{const}(n, k, m, \lambda, \Lambda, \vartheta)$.

Proof. We assume that (5.1) is satisfied with parameters $\epsilon, \gamma$ to be specified later, $0<\sigma \leq 1, \chi \leq 1$, and $E \leq 1$. Since (5.1) implies the smallness condition (2.1) in the hypotheses of Lemma 2.2 (by the choice of $C_{17}$ ), we can use the height estimate

$$
\sup \left\{|\mathbf{q} x|: x \in \mathbf{C}_{1 / 2} \cap \operatorname{spt} T\right\} \leq \operatorname{const}(n, \Lambda) E^{1 / 2 n}
$$

Letting $S=\operatorname{graph}(L)$ as in Lemma 5.1 we see from (5.3) that there is a linear isometry, of $\mathbb{R}^{n+k}$ satisfying, $S=\mathbb{R}^{n} \times\{0\}^{k},=\mathrm{id}$ on $\left(\mathbb{R}^{n-1} \times\{0\}\right) \times\{0\}^{k}$ in the case $T \in \mathcal{T}_{b}(n, k, m)$, and

$$
\|,-\operatorname{id}\| \leq \operatorname{const}(n, m, \lambda, \Lambda) \sqrt{E+\sigma^{-1} \chi}
$$


Assuming $0<\eta \leq \frac{1}{17}$ and a smallness condition

$$
C_{19}\left(E^{1 / 2 n}+\sqrt{E+\sigma^{-1} \chi}\right) \leq \eta
$$

with a sufficiently large constant $C_{19}=\operatorname{const}(n, m, \lambda, \Lambda)$, we verify by elementary geometric considerations, using the height bound (5.15) in $\mathbf{C}_{1 / 2}$, the height bound 1 in $\mathbf{C} \backslash \mathbf{C}_{1 / 2},(5.16)$, and (5.4), that

$$
\left(,{ }^{-1} \mathbf{C}_{\eta / 4}\right) \cap \operatorname{spt} T \subset \mathbf{C}_{\eta / 2}
$$

and

$$
\mathbf{C}_{\eta / 4} \cap \boldsymbol{\tau}_{w}^{-1}(, \operatorname{spt} T) \subset \mathbf{U}_{\eta / 4}^{n} \times \mathbf{B}^{k}(0, \eta)
$$

where $w=, v$ and $v \in S^{\perp}$ is from Lemma 5.1. The conclusion (5.2) of this Lemma and (5.15) imply

$$
\begin{aligned}
& \int_{\mathbf{C}_{\eta / 4}}|\mathbf{q} x-w|^{2} d\|, \# T\| x=\int_{\Gamma^{-1} \mathbf{C}_{\eta / 4}} \operatorname{dist}(x-v, S)^{2} d\|T\| x \\
& \leq \int_{\mathbf{C}_{\eta / 2}} \operatorname{dist}(x-v, S)^{2} d\|T\| x \leq C_{18}\left(\gamma^{-2} E^{1 / n}+\eta^{n+4}+\epsilon\right)\left(E+\sigma^{-1} \chi\right) .
\end{aligned}
$$

We next apply the reverse Poincaré inequality Lemma 4.2 to the scaled current

$$
\tilde{T}=\boldsymbol{\mu}_{\eta / 4 \#}^{-1} \boldsymbol{\tau}_{w \#}^{-1}\left(, \# T\left\llcorner\mathbf{C}_{\eta / 4}\right)\right.
$$

Since $(\partial \tilde{T})\llcorner\mathbf{C}=(\partial T)\llcorner\mathbf{C}$ it is easy to see, by a homotopy connecting, to the identity, that $\tilde{T}$ satisfies all the requirements from the definition of the class $\mathcal{T}_{b}(n, k, 1)$ or $\mathcal{T}_{i}(n, k, m)$ respectively (including $(2.12)$ ) with the exception $0 \in \operatorname{spt} \tilde{T}$ in the latter case. (This condition, however, is not needed in Lemma 4.2 as we have pointed out there.) $\tilde{T}$ is $(\tilde{F}, \tilde{\omega})$ minimizing in $\mathbf{C}$ with $\tilde{\omega}(r)=\omega\left(\frac{\eta}{4} r\right)$, structure constants $\tilde{\lambda}=\lambda, \tilde{\Lambda}=\Lambda$, and moduli $\tilde{\kappa}(r)=\kappa\left(\frac{\eta}{4} r\right), \tilde{\mu}(r)=\mu\left(\frac{\eta}{4} r\right), \widehat{\tilde{\mu}}(r) \leq \widehat{\mu}\left(\frac{\eta}{4} r\right)$. The height $h$ of $\tilde{T}$ in $\mathbf{C}$ does not exceed 4, by (5.19), hence $\tilde{\omega}\left(\sqrt{1+h^{2}}\right) \leq \omega\left(\frac{1}{2}\right)$ and similarly for $\tilde{\kappa}, \tilde{\mu}, \widehat{\tilde{\mu}}$. The conclusion of Lemma 4.2 therefore gives

$$
\begin{aligned}
\mathbf{E}\left(,{ }_{\#} T, \frac{1}{20} \eta\right) \leq C_{12}\left[\chi ( \frac { \eta } { 4 } ) ^ { - n } \mathbf { M } \left(, \ldots T\left\llcorner\mathbf{C}_{\eta / 4}\right)\right.\right. & \\
& \left.+(1+\chi)\left(\frac{\eta}{4}\right)^{-n-2} \int_{\mathbf{C}_{\eta / 4}}|\mathbf{q} x-w|^{2} d\|, \# T\| x\right],
\end{aligned}
$$

and combining this with (5.20) we get

$$
\mathbf{E}\left({ }_{\#} T, \frac{1}{20} \eta\right) \leq C_{20}\left[\chi \eta^{-n}+\left(\eta^{-n-2} \gamma^{-2} E^{1 / n}+\eta^{2}+\eta^{-n-2} \epsilon\right)\left(E+\sigma^{-1} \chi\right)\right]
$$

where $C_{20}=\operatorname{const}(n, m, \lambda, \Lambda)$ and we have used

$$
\mathbf{M}\left({ }_{\#} T\left\llcorner\mathbf{C}_{\eta / 4}\right) \leq \mathbf{M}\left(T\left\llcorner\mathbf{C}_{\eta / 2}\right) \leq E+m \boldsymbol{\alpha}(n) .\right.\right.
$$


We now specify $\eta=20 \vartheta$ with $0<\vartheta \leq \frac{1}{340}, \epsilon=\eta^{n+2} \vartheta^{2}, \sqrt{\sigma}=\frac{1}{2} C_{17}^{-1} \delta(n, k, \lambda, \Lambda, \epsilon)$, and $\gamma=E^{1 / 4 n}$. If we require

$$
E^{1 / 2 n} \leq \vartheta^{n+4}
$$

then (5.21) gives the desired conclusion (5.14). Moreover, with these specifications of $\epsilon$ and $\gamma$ all the smallness assumptions (5.1), (5.17), and (5.22) are satisfied if we require (5.12) with a sufficiently small constant $\delta_{1}$. (5.13) finally follows from (5.3).

We note that in the boundary case $T \in \mathcal{T}_{b}(n, k, m)$ with $(2.13)$ and multiplicity $m \geq 2$ the proof above does not work, because it is not clear that the tilted current $\tilde{T}$ does again satisfy the density condition (2.13) which we needed for the height estimate. Thus, here is a second difficulty (besides that pointed out after Lemma 5.1) which prevents us from proving boundary regularity in the case of multiplicity $m \geq 2$. It seems that one would need an estimate of the height of $\tilde{T}$ by the distance to spt $\partial T$ to overcome this difficulty.

We now iterate Lemma 5.2. We consider $0<\vartheta \leq \frac{1}{340}$ such that $C_{22} \vartheta^{2}<\frac{1}{2} \vartheta$. With $\delta_{1}$ from (5.12) we choose positive numbers $E_{*} \leq \delta_{1}$ and $\chi_{*} \leq \delta_{1}$. Starting with $T_{0} \in \mathcal{T}_{b}(n, k, 1)$ or $T_{0} \in \mathcal{T}_{i}(n, k, m)$ satisfying (2.12) such that spt $T_{0} \subset \mathbb{R}^{n} \times \mathbf{B}^{k}$ and $T_{0}$ is $\left(F_{0}, \omega_{0}\right)$ minimizing in $\mathbf{C}$ for some integrand $F_{0}$ with structure constants resp. moduli $\lambda_{0}=\lambda, \Lambda_{0}=\Lambda, \nu_{0}=\nu$, $\kappa_{0} \leq \kappa, \mu_{0} \leq \mu$ and for some nondecreasing $\omega_{0} \leq \omega$, where $\kappa_{0}, \kappa, \mu_{0}, \mu, \omega_{0}, \omega$ satisfy the conditions of Section 1, we assume

$$
\mathbf{E}\left(T_{0}, 1\right) \leq E_{*}, \quad \chi_{0}(1) \leq \chi_{*}
$$

Here we define

$$
\chi(r)=\left\{\begin{array}{cl}
\widehat{\kappa}(r)+\omega(r) & \text { in the case } T \in \mathcal{T}_{i}(n, k, m), \\
\kappa(r)^{2}+\widehat{\mu}(r)+\omega(r) & \text { in the case } T_{0} \in \mathcal{T}_{b}(n, k, 1) .
\end{array}\right.
$$

The function $\chi_{0}(r)$ is defined similarly using $\kappa_{0}, \widehat{\kappa_{0}}, \widehat{\mu_{0}}$, and $\omega_{0}$. We may then apply Lemma 5.2 to find a linear isometry, 0 of $\mathbb{R}^{n+k}$, with , $0=$ id on $\left(\mathbb{R}^{n-1} \times\{0\}\right) \times\{0\}^{k}$ in the case $T_{0} \in \mathcal{T}_{b}(n, k, 1)$, such that

$$
\|, 0-\mathrm{id}\| \leq C_{21} \sqrt{\mathbf{E}\left(T_{0}, 1\right)+\chi_{0}(1)}
$$

and

$$
\mathbf{E}\left(T_{1}, 1\right) \leq \eta \mathbf{E}\left(T_{0}, 1\right)+C_{21} \chi_{0}(1)
$$

hold for $T_{1}=\left(\boldsymbol{\mu}_{\vartheta \#}^{-1}, 0 \# T_{0}\right)\left\llcorner\mathbf{C}\right.$ and $\eta=C_{22} \vartheta^{2}<\frac{1}{2} \vartheta$. Moreover, $T_{1}$ is $\left(F_{1}, \omega_{1}\right)$ minimizing in $\mathbf{C}$ for an integrand $F_{1}$ satisfying the same conditions as $F_{0}$ with constants and moduli $\lambda_{1}=\lambda, \Lambda_{1}=\Lambda, \nu_{1}=\nu, \kappa_{1}(r) \leq \kappa(\vartheta r), \widehat{\kappa_{1}}(r) \leq \widehat{\kappa}(\vartheta r), \omega_{1}(r) \leq \omega(\vartheta r)$, and in the boundary case also $\mu_{1}(r) \leq \mu(\vartheta r), \widehat{\mu_{1}}(r) \leq \widehat{\mu}(\vartheta r)$, i.e. $\chi_{1}(r) \leq \chi(\vartheta r)$. Hence, making use of (5.26) we see that (5.23) holds for $T_{1}, \chi_{1}(1)$ provided

$$
C_{21} \chi_{*} \leq(1-\eta) E_{*} .
$$

If we impose an additional smallness condition

$$
C_{23} E_{*} \leq \vartheta^{2 n}
$$


with sufficiently large constant $C_{23}=\operatorname{const}(n, k, m, \lambda, \Lambda)$, then the height estimate Lemma 2.2 and (5.25) imply also the height condition spt $T_{1} \subset \mathbb{R}^{n} \times \mathbf{B}^{k}$ for $T_{1}$.

Thus, $T_{1}$ satisfies the same set of conditions as $T_{0}$ and we can proceed to obtain $T_{i}, \chi_{i}$, ,$i$ such that

$$
\begin{aligned}
& T_{i+1}=\left(\boldsymbol{\mu}_{\vartheta \#}^{-1}, i \# T_{i}\right)\left\llcorner\mathbf{C}, \quad \chi_{i}(r) \leq \chi\left(\vartheta^{i} r\right),\right. \\
& \left\|,{ }_{i}-\mathrm{id}\right\| \leq C_{21} \sqrt{\mathbf{E}\left(T_{i}, 1\right)+\chi_{i}(1)}
\end{aligned}
$$

and

$$
\mathbf{E}\left(T_{i+1}, 1\right) \leq \eta \mathbf{E}\left(T_{i}, 1\right)+C_{21} \chi_{i}(1)
$$

From (5.30) and (5.29) we deduce

$$
\mathbf{E}\left(T_{i}, 1\right) \leq \eta^{i} \mathbf{E}\left(T_{0}, 1\right)+C_{21} \sum_{j=1}^{i} \eta^{j-1} \chi\left(\vartheta^{i-j}\right)
$$

To simplify this we use the assumption that $r^{-2 \alpha} \kappa(r)^{2}, r^{-2 \alpha} \widehat{\kappa}(r), r^{-2 \alpha} \widehat{\mu}(r), r^{-2 \alpha} \omega(r)$, hence also $r^{-2 \alpha} \chi(r)$, are nonincreasing for some $0<\alpha<1$. Therefore $\chi\left(\vartheta^{i-j}\right) \leq \vartheta^{-2 \alpha j} \chi\left(\vartheta^{i}\right)$ in the last sum and, consequently,

$$
\mathbf{E}\left(T_{i}, 1\right) \leq \eta^{i} \mathbf{E}\left(T_{0}, 1\right)+2 C_{21} \vartheta^{-2 \alpha} \chi\left(\vartheta^{i}\right),
$$

provided we have chosen $\eta=C_{22} \vartheta^{2} \leq \frac{1}{2} \vartheta^{2 \alpha}$.

We next want to prove that ${ }_{i}=,{ }_{i},{ }_{i-1} \ldots, 0$ converges to some limit isometry , $\infty$. From (5.29) and (5.31) we have

$$
\left\|\sim_{i}-\sim_{i-1}\right\|=\left\|,{ }_{i}-\mathrm{id}\right\| \leq C_{21}\left[\eta^{i} \mathbf{E}\left(T_{0}, 1\right)+\left(2 C_{21} \vartheta^{-2 \alpha}+1\right) \chi\left(\vartheta^{i}\right)\right]^{1 / 2} .
$$

It follows that $\sum_{i=1}^{\infty}\left\|_{,_{i}}^{\sim} \tilde{\sim}_{i-1}\right\|$ converges, provided $\sum_{i=1}^{\infty} \sqrt{\chi\left(\vartheta^{i}\right)}<\infty$. In view of

$$
\frac{1}{\alpha}\left(1-\vartheta^{\alpha}\right) \sqrt{\chi\left(\vartheta^{i}\right)}=\frac{\sqrt{\chi\left(\vartheta^{i}\right)}}{\vartheta^{\alpha i}} \int_{\vartheta^{i+1}}^{\vartheta^{i}} r^{\alpha-1} d r \leq \int_{\vartheta^{i+1}}^{\vartheta^{i}} \frac{1}{r} \sqrt{\chi(r)} d r
$$

the convergence of $\sum_{i=1}^{\infty} \sqrt{\chi\left(\vartheta^{i}\right)}$ is implied by the condition (cf. (1.15))

$$
\infty>\mathrm{X}(r)=\left\{\begin{array}{cl}
\widehat{\mathrm{K}}(r)+\Omega(r) & \text { in the case } T \in \mathcal{T}_{i}(n, k, m), \\
\mathrm{K}(r)^{2}+\widehat{\mathrm{M}}(r)+\Omega(r) & \text { in the case } T_{0} \in \mathcal{T}_{b}(n, k, 1) .
\end{array}\right.
$$

Assuming (5.33) we obtain a limit, $\infty$ of the ${ }^{\sim}{ }_{i}$ with the estimate (recalling $\eta \leq \frac{1}{2} \vartheta^{2 \alpha} \ll 1$ )

$$
\|, \infty-\stackrel{\sim}{i-1}_{i-1} \leq 2 C_{21}\left[\sqrt{\eta^{i} \mathbf{E}\left(T_{0}, 1\right)}+\frac{\alpha}{1-\vartheta^{\alpha}} \sqrt{2 C_{21} \vartheta^{-2 \alpha}+1} \sqrt{\mathrm{X}\left(\vartheta^{i}\right)}\right]
$$


where $i=0$ gives an estimate for $\|, \infty-\mathrm{id}\|$. Imposing a further smallness condition

$$
C_{24}\left(\sqrt{E_{*}}+\sqrt{\mathrm{X}_{*}}\right) \leq 1
$$

with a sufficiently large constant $C_{24}=\operatorname{const}(n, k, m, \lambda, \Lambda, \vartheta)$ and assuming $\mathbf{X}(1) \leq \mathrm{X}_{*}$, we see from (5.34) and spt $T_{i} \subset \mathbb{R}^{n} \times \mathrm{B}^{k}$ that

$$
\left(, \infty \# \stackrel{\sim}{\sim} \underset{i \#}{-1} T_{i+1}\right)\left\llcorner\mathbf{C}_{\vartheta}=\left(\boldsymbol{\mu}_{\vartheta}^{-1}, \infty \# \stackrel{\sim}{-1}{ }_{i-1 \#} T_{i}\right)\left\llcorner\mathbf{C}_{\vartheta}\right.\right.
$$

This implies, by induction,

$$
\mathbf{E}\left(,{ }_{\infty \#,}^{\sim}{ }_{i-1 \#}^{-1} T_{i}, \vartheta\right)=\mathbf{E}\left(, \infty \# T_{0}, \vartheta^{i+1}\right) .
$$

We now invoke the following simple Lemma:

5.3 Lemma (excess comparison for tilting). Suppose $T \in \mathcal{T}_{*}(n, k, m)$ with spt $T \subset$ $\mathbb{R}^{n} \times \mathbf{B}^{k}$ and, is a linear isometry of $\mathbb{R}^{n+k}$ with, $=\mathrm{id}$ on $\left(\mathbb{R}^{n} \times\{0\}\right) \times\{0\}^{k}$ in the case $T \in \mathcal{T}_{b}(n, k, m)$. If $\|,-\mathrm{id}\| \leq \frac{1}{5}$, then

$$
\varrho^{n} \mathbf{E}(, \# T, \varrho) \leq 2 \mathbf{E}(T, 1)+n^{2}\|,-\mathrm{id}\|^{2} \mathbf{M}(T)
$$

holds for $0<\varrho \leq \frac{2}{3}$.

Proof. The hypotheses imply $\left(,{ }^{-1} \mathbf{C}_{\varrho}\right) \cap \operatorname{spt} T \cap \partial \mathbf{C}=\emptyset$, and with a homotopy argument one sees that $\mathbf{p}_{\#}\left(\right.$, \#T $T\left\llcorner\mathbf{C}_{\varrho}\right)=\left(\mathbf{E}^{n}\left\llcorner\mathbf{U}_{\varrho}\right)\left\llcorner\iota_{m}\right.\right.$ with the same nonnegative multiplicity function $\iota_{m}$ as in $\mathbf{p}_{\#} T=\left(\mathbf{E}^{n}\llcorner\mathbf{U})\left\llcorner\iota_{m}\right.\right.$. Therefore

$$
\begin{aligned}
\varrho^{n} \mathbf{E}\left(,{ }_{\#} T, \varrho\right) & =\frac{1}{2} \int_{\mathbf{C}_{\varrho}}\left|\overrightarrow{{ }_{\#} T}-\overrightarrow{\mathbf{e}}\right|^{2} d\left\|,{ }_{\#} T\right\| \leq \frac{1}{2} \int_{\mathbf{C}}\left|\left(\bigwedge_{n},\right) \vec{T}-\overrightarrow{\mathbf{e}}\right|^{2} d\|T\| \\
& \leq \int_{\mathbf{C}}|\vec{T}-\overrightarrow{\mathbf{e}}|^{2} d\|T\|+\int_{\mathbf{C}}\left|\left(\bigwedge_{n},\right) \vec{T}-\vec{T}\right|^{2} d\|T\| \\
& \leq 2 \mathbf{E}(T, 1)+n^{2}\|,-\mathrm{id}\|^{2} \mathbf{M}(T) .
\end{aligned}
$$

Applying this with $=, \sim_{i-1}^{-1}$ and $T=T_{i}$, and noting that (5.34), (5.35) imply $\|,-\mathrm{id}\| \leq \frac{1}{5}$ if $C_{24}$ is chosen large enough, we deduce

$$
\mathbf{E}\left(, \infty \# T_{0}, \vartheta^{i+1}\right) \leq 2 \vartheta^{-n} \mathbf{E}\left(T_{i}, 1\right)+\operatorname{const}(n, m)\left\|, \infty-\sim_{i-1}\right\|^{2},
$$

and with (5.31), (5.36), (5.34) we obtain, noting that $\chi\left(\vartheta^{i}\right) \leq \operatorname{const}(\vartheta) \times\left(\vartheta^{i}\right)$ by $(5.32)$,

$$
\mathbf{E}\left(,{ }_{\infty \#} T_{0}, \vartheta^{i+1}\right) \leq C_{25}\left[\eta^{i} \mathbf{E}\left(T_{0}, 1\right)+\mathbf{X}\left(\vartheta^{i}\right)\right]
$$

This is the discrete version of the excess decay for $T_{0}$ over the tilted $n$-plane,${ }_{\infty}^{-1}\left(\mathbb{R}^{n} \times\{0\}^{k}\right)$. The following Lemma is an immediate consequence. We recall the definition of $\mathbf{X}$ in (5.33) and the assumed existence of $0<\alpha<1$ such that $r^{-\alpha} \kappa(r), r^{-\alpha} \mu(r), r^{-2 \alpha} \omega(r)$, hence also $r^{-2 \alpha} \widehat{\kappa}(r), r^{-2 \alpha} \widehat{\mu}(r)$ and $r^{-2 \alpha} \chi(r)$, are nonincreasing. 
5.4 Lemma (excess decay). For $\alpha \leq \beta<1$ there exists a positive constant $\epsilon_{0}=$ $\epsilon_{0}(n, k, m, \lambda, \Lambda, \nu(\cdot), \beta)$ such that the following is true: Whenever $T \in \mathcal{T}_{b}(n, k, 1)$ or $T \in$ $\mathcal{T}_{i}(n, k, m)$ with (2.12) is $(F, \omega)$ minimizing in $\mathbf{C}$ with

$$
\mathbf{E}(T, 1)+\mathrm{X}(1) \leq \epsilon_{0}
$$

then there exists a linear isometry, of $\mathbb{R}^{n+k}$ with, $=\mathrm{id}$ on $\left(\mathbb{R}^{n-1} \times\{0\}\right) \times\{0\}^{k}$ in the case $T \in \mathcal{T}_{b}(n, k, 1)$ such that

$$
\|,-\mathrm{id}\|^{2} \leq C_{26}[\mathbf{E}(T, 1)+\mathrm{X}(1)]
$$

and

$$
\begin{aligned}
& \mathbf{E}\left(, \#\left(T\left\llcorner\mathbf{C}_{1 / 2}\right), r\right) \leq C_{26}\left[r^{2 \beta} \mathbf{E}(T, 1)+\mathbf{X}(r)\right] \text { for } 0<r \leq \frac{1}{3},\right. \\
& \mathbf{E}(T, r) \leq C_{26}[\mathbf{E}(T, 1)+\mathbf{X}(1)] \text { for } 0<r \leq 1,
\end{aligned}
$$

where $C_{26}=\operatorname{const}(n, k, m, \lambda, \Lambda, \beta)$.

Proof. We define $T_{0}=\boldsymbol{\mu}_{2 \#}\left(T\left\llcorner\mathbf{C}_{1 / 2}\right)\right.$ and observe that $T_{0}$ is $\left(F_{0}, \omega_{0}\right)$ minimizing with $\lambda_{0}=\lambda, \Lambda_{0}=\Lambda, \nu_{0}=\nu, \chi_{0} \leq \chi\left(\frac{1}{2} r\right)$, for $\chi$ defined in (5.24) and $\chi_{0}$ analogously. Clearly, we may replace $\alpha$ by any larger exponent in ]0,1[ and hence assume $\alpha=\beta$. We define $\vartheta$ depending on $n, m, \lambda, \Lambda$, and $\alpha$ by $\vartheta=\min \left\{\left(2 C_{22}\right)^{-1 /(2-2 \alpha)}, \frac{1}{340}\right\}$ so that $C_{22} \leq \frac{1}{2} \vartheta^{2 \alpha-2}$ and $\eta=C_{22} \vartheta^{2} \leq \frac{1}{2} \vartheta^{2 \alpha}$. Choosing $\epsilon_{0}$ small enough we see that all our previous smallness conditions (5.23), (5.27), (5.28), and (5.35) are satisfied, and spt $T_{0} \subset \mathbb{R}^{n} \times \mathrm{B}^{k}$ holds by Lemma 2.2. We let, be the limit isometry, $\infty$ produced above. Then (5.39) follows from (5.34) and $\mathbf{E}\left(T_{0}, 1\right) \leq 2^{n} \mathbf{E}(T, 1)$.

For $\vartheta^{i+2}<r \leq \vartheta^{i+1}$ with $i \geq 0$ we obtain from (5.37)

$$
\begin{aligned}
\mathbf{E}\left(,_{\#} T_{0}, r\right) & \leq \vartheta^{-n} \mathbf{E}\left(,{ }_{\#}, \vartheta^{i+1}\right) \leq \vartheta^{-n} C_{25}\left[\eta^{i} \mathbf{E}\left(T_{0}, 1\right)+\mathrm{X}\left(\vartheta^{i}\right)\right] \\
& \leq \vartheta^{-n} C_{25}\left[\vartheta^{-4 \alpha} r^{2 \alpha} \mathbf{E}\left(T_{0}, 1\right)+\mathrm{X}\left(\vartheta^{-2} r\right)\right] .
\end{aligned}
$$

This implies (5.40) for $0<r \leq \frac{1}{2} \vartheta$, because we have $\mathbf{X}(t \varrho) \leq t^{2 \alpha} \mathbf{X}(\varrho)$ for $t \geq 1$ and $\mathbf{E}\left({ }_{\#}\left(T\left\llcorner\mathbf{C}_{1 / 2}\right), \frac{1}{2} r\right)=\mathbf{E}\left(,_{\#} T_{0}, r\right)\right.$. For $\frac{1}{2} \vartheta<r \leq \frac{1}{3}$ we deduce (5.40) from (5.39) with Lemma 5.3, decreasing $\epsilon_{0}$ if necessary.

With regard to (5.41) we note that by Lemma 2.2, (5.38), (5.39), and (5.40) we have the following, provided $\epsilon_{0}$ is chosen sufficiently small:

$$
\begin{aligned}
& \widetilde{T}_{r}=\left[\boldsymbol{\mu}_{r \#}^{-1}, \#\left(T\left\llcorner\mathbf{C}_{1 / 2}\right)\right]\left\llcorner\mathbf{C} \in \mathcal{T}_{*}(n, k, m),\right.\right. \\
& \sup \left\{|\mathbf{q} x|: x \in \operatorname{spt} \widetilde{T}_{r}\right\} \leq 1, \\
& \|,-\operatorname{id}\|^{2} \leq C_{26}[\mathbf{E}(T, 1)+\mathbf{X}(1)] \leq \frac{1}{25}, \\
& \mathbf{E}\left(\widetilde{T}_{r}, 1\right) \leq C_{26}[\mathbf{E}(T, 1)+\mathbf{X}(1)], \\
& \left(,{ }_{\#}^{-1} \widetilde{T}_{r}\right)\left\llcorner\mathbf{C}_{1 / 2}=\left(\boldsymbol{\mu}_{r \#}^{-1} T\right)\left\llcorner\mathbf{C}_{1 / 2},\right.\right.
\end{aligned}
$$


for $0<r \leq \frac{1}{8}$. With Lemma 5.3 and $\mathbf{M}\left(\widetilde{T}_{r}\right) \leq \mathbf{E}\left(\widetilde{T}_{r}, 1\right)+m \boldsymbol{\alpha}(n)$ we conclude

$$
\begin{aligned}
\mathbf{E}\left(T, \frac{r}{2}\right) & =\mathbf{E}\left(\boldsymbol{\mu}_{r \#}^{-1} T, \frac{1}{2}\right)=\mathbf{E}\left(, \underset{\#}{-1} \widetilde{T}_{r}, \frac{1}{2}\right) \\
& \leq \operatorname{const}(n, m)\left[\mathbf{E}\left(\widetilde{T}_{r}, 1\right)+\left\|,{ }^{-1}-\mathrm{id}\right\|^{2}\right] \\
& \leq 2 \operatorname{const}(n, m) C_{26}[\mathbf{E}(T, 1)+\mathbf{X}(1)] .
\end{aligned}
$$

Increasing $C_{26}$ suitably we have therefore proved (5.41) for $0<r \leq \frac{1}{16}$, and for $\frac{1}{16} \leq r \leq 1$ it holds trivially, by the scaling property of the excess, provided $C_{26} \geq 16^{n}$.

\section{The $\epsilon$-regularity theorem}

In this section we use the excess decay (Lemma 5.4) to prove the $\epsilon$-regularity theorem: An $(F, \omega)$ minimizing current $T$ with sufficiently small excess in arbitrarily small balls centered at $a \in \operatorname{spt} T$ is, locally at $a$, represented by a $C^{1}$ submanifold (with boundary spt $\partial T$ if $\partial T$ is, locally at $a$, represented by a smooth submanifold). It is well known how to deduce this result from the excess decay (e.g. [All2], [Ha1]), but we give a complete proof, in order to controll the dependence of constants and to derive an explicit modulus of continuity for the first oder derivatives.

We assume here all the structure conditions from Section 1 on the integrand $F$ and the function $\omega$ in the condition of $(F, \omega)$ minimality. In particular, $\lambda>0$ is the ellipticity constant of $F, \Lambda$ is a bound for $F(x, \xi)$ and its derivatives up to order 2 with respect to the tangent plane variable $\xi, \nu(\cdot)$ is a modulus of continuity for this second derivative, $\kappa, \mu$ are moduli for $F(x, \xi)$ and $D_{(2)} F(x, \xi)$ with respect to the space variable $x$ as in $(1.4)-(1.8), \widehat{\kappa}$, $\widehat{\mu}$ are associated with $\kappa, \mu$ by (1.9), and $\mathrm{K}, \widehat{\mathrm{K}}, \widehat{\mathrm{M}}, \Omega$ are defined in (1.15). The exponent $0<\alpha<1$ is such that $r^{-\alpha} \kappa(r), r^{-\alpha} \mu(r), r^{-2 \alpha} \omega(r)$ are nonincreasing functions of $r>0$. Recall also the density condition (2.12), i.e. $\Theta^{n}(\|T\|, x) \geq m$ for $\|T\|$ almost all $x$, in the case $T \in \mathcal{T}_{i}(n, k, m)$ with $m \geq 2$. In the boundary situation $T \in \mathcal{T}_{b}(n, k, m)$ we must assume $m=1$ for reasons explained in section 5 . $\mathbf{E}(T, 1)$ is the cylindrical excess of $T$ in the cylinder $\mathbf{C}=\mathbf{U}^{n} \times \mathbb{R}^{k} \subset \mathbb{R}^{n+k}$.

6.1 Theorem (interior and boundary $\epsilon$-regularity theorem). Corresponding to $\alpha \leq \beta<1$ there exists a positive constant $\epsilon(n, k, m, \lambda, \Lambda, \nu(\cdot), \beta)$ such that the following is true: If $T \in \mathcal{T}_{i}(n, k, m)$ with (2.12) or $T \in \mathcal{T}_{b}(n, k, m)$ with $m=1$ is $(F, \omega)$ minimizing in C with

$$
\mathbf{E}(T, 1)+\widehat{\mathrm{K}}(1)+\Omega(1) \leq \epsilon,
$$

then $T\left\llcorner\mathbf{C}_{1 / 34}\right.$ is represented by the graph, taken with multiplicity $m$, of a continuously differentiable function $g$ which is defined over the $n$-disk $\mathbf{U}_{1 / 34}$ in the case $T \in \mathcal{T}_{i}(n, k, m)$ and over the half $n$-disk $\mathbf{B}_{+} \cap \mathbf{U}_{1 / 34}$ in the case $T \in \mathcal{T}_{b}(n, k, m), m=1$. Moreover, the derivative $\mathrm{Dg}$ has the following modulus of continuity:

$$
|D g(a)-D g(b)| \leq C_{33}\left[|a-b|^{2 \beta} \mathbf{E}(T, 1)+\widehat{\mathrm{K}}(|a-b|)+\Omega(|a-b|)\right]^{1 / 2}
$$

for $a, b$ in the domain of $g$, where $C_{33}=\operatorname{const}(n, k, m, \lambda, \Lambda, \beta)$. 
Since one can always flatten the boundary by a diffeomorphism and achieve smallness of $\widehat{\mathrm{K}}(1)$ and $\Omega(1)$ by scaling, Theorem 6.1 can be applied to $(F, \omega)$ minimizing rectifiable $n$ currents $T$ in $\mathbb{R}^{n+k}$, with $F$ sufficiently regular, at all points $a \in \operatorname{spt} T$ where $\mathbf{E}\left(T, a, \varrho_{i}\right)<\frac{1}{2} \epsilon$ for a sequence $\varrho_{i} \downarrow 0$ and where, in the case $a \in \operatorname{spt} \partial T$, $\partial T$ is represented locally by an oriented $C^{1,1}$ submanifold of dimension $n-1$ in $\mathbb{R}^{n+k}$ an $\mathbf{p}_{\#} T$ has multiplicity 0 and 1 on the two sides of the projection of spt $\partial T$ near $a$. In this situation the theorem gives interior and boundary $C^{1, \beta}$ regularity of $T$ locally at 0 for all $0<\beta<1$ when $\Omega \equiv 0$ (i.e. $T$ is $F$ minimizing), and regularity with continuity modulus const $\Omega$ for the derivative when $\Omega \not \equiv 0$ (which is optimal in the case $\omega(r)=$ const $r^{\beta}$, cf. Section 1 ). The radius $\frac{1}{17}$ of the domain of $g$ can, of course, be replaced by any radius $0<r<1$ (if one admits dependence of $\epsilon$ and $C_{33}$ on $r$ ). This is easily seen from the scaling properties of the excess and the functions $\widehat{\mathrm{K}}(r), \Omega(r)$.

Proof. We require $\epsilon<\epsilon_{0}$ with $\epsilon_{0}(n, k, m, \lambda, \Lambda, \nu(\cdot), \beta)$ from Lemma 5.4, and we will impose further smallness conditions on $\epsilon$ in the course of the proof.

Step 1. We prove that the excess of $T$ is uniformly small in all cylinders $\mathbf{C}_{r}(y) \subset \mathbf{C}$ with $y \in \mathbf{B}_{1 / 2}$ and in the boundary case additionally $y \in \mathbf{B}_{+}$.

In the interior situation $T \in \mathcal{T}_{i}(n, k, m)$ we observe $\mathbf{E}\left(T, y, r_{0}\right) \leq 2^{n} \mathbf{E}(T, 0,1) \leq 2^{n} \epsilon$ for $r_{0}=1-|y|$, hence choosing $\epsilon \leq 2^{-n-1} \epsilon_{0}$ we see that the scaled current $\left(\boldsymbol{\mu}_{r_{0} \#}^{-1} \boldsymbol{\tau}_{x \#}^{-1} T\right)\llcorner\mathbf{C}$ with $x \in \mathbf{p}^{-1}\{y\} \cap \operatorname{spt} T$ satisfies the hypotheses of Lemma 5.4. From (5.41) we therefore get a bound const $\cdot \epsilon$ on $\mathbf{E}(T, y, r)$ for all $0<r \leq r_{0}$, and decreasing $\epsilon$ we achieve

$$
\mathbf{E}(T, y, r)+\widehat{\mathrm{K}}(r)+\Omega(r) \leq \operatorname{const}(n, k, m, \lambda, \Lambda, \beta) \epsilon \leq \epsilon_{0}
$$

for all $y \in \mathbf{B}_{1 / 2}$ and $0<r \leq 1-|y|$.

In the boundary situation we apply Lemma 5.4 similarly, but estimate $\mathrm{K}(r)^{2}+\widehat{\mathrm{M}}(r) \leq$ $5 \widehat{\mathrm{K}}(r)$ for $0<r \leq \frac{1}{2}$, which follows from $\frac{1}{4} \kappa(\varrho)^{2} \leq \widehat{\kappa}(\varrho)$ and $\widehat{\mu}(\varrho) \leq \widehat{\kappa}(\varrho)$ for $0<\varrho \leq \frac{1}{2}$. We then obtain (6.3) for $T \in \mathcal{T}_{b}(n, k, 1)$ and centers $y \in \mathbf{B}_{1 / 2}^{n-1} \times\{0\}$. If $y \in \mathbf{U}_{+} \cap \mathbf{B}_{1 / 2}$ then we consider $y^{\prime}=\left(y_{1}, \ldots, y_{n-1}, 0\right) \in \mathbf{B}_{1 / 2}^{n-1} \times\{0\}$ and $\left.\left.r_{0}=y_{n} \in\right] 0, \frac{1}{2}\right]$. In the case $r_{0} \geq \frac{1}{4}$ we deduce (6.3) for $0<r \leq 1-|y|$ as above. In the case $r_{0}<\frac{1}{4}$ we have $\mathbf{U}_{r}(y) \subset \mathbf{U}_{2 r}\left(y^{\prime}\right) \subset \mathbf{U}$ for $r_{0} \leq r \leq \frac{1}{4}$, and using the smallness of $\mathbf{E}\left(T, y^{\prime}, 2 r\right)$ we deduce again a corresponding estimate for $\mathbf{E}(T, y, r), r_{0} \leq r \leq \frac{1}{4}$. For $0<r<r_{0}$ a similar estimate follows from the smallness of $\mathbf{E}\left(T, y, r_{0}\right)$ by the scaling argument above and Lemma 5.4.

Replacing $T$ by $\boldsymbol{\mu}_{2 \#}\left(T\left\llcorner\mathbf{C}_{1 / 2}\right)\right.$ and choosing $\epsilon$ small enough we may therefore assume that (6.3) holds for all $y \in \mathbf{U}$ and $0<r \leq 1-|y|$, where $y_{n} \geq 0$ is required additionally in the boundary case. (In Theorem 6.1 we have stated the conclusion for the radius $\frac{1}{34}$ instead of $\varrho=\frac{1}{17}$, because we have expanded $T$ by a factor 2.) This means that Lemma 5.4 can be applied to all the rescaled currents $\left(\boldsymbol{\mu}_{r \#}^{-1} \boldsymbol{\tau}_{x \#}^{-1} T\right)\left\llcorner\mathbf{C}\right.$ with $x \in \mathbf{p}^{-1}\{y\} \cap \operatorname{spt} T$, $0<r \leq 1-|y|$, and in the boundary case additionally $x=(y, 0)$ or $\mathbf{U}_{r}(y) \subset \mathbf{U}_{+}$. By Lemma 2.2 we may also assume that these currents have height $\leq 1$, i.e. support in $\mathbb{R}^{n} \times \mathbf{B}^{k}$.

Step 2. We prove that $\mathbf{C}_{1 / 17} \cap \operatorname{spt} T$ is the graph of a Lipschitz function defined on $\mathbf{U}_{1 / 17}$ in the case $T \in \mathcal{T}_{i}(n, k, m)$ and on $\mathbf{B}_{+} \cap \mathbf{U}_{1 / 17}$ in the case $T \in \mathcal{T}_{b}(n, k, 1)$. Moreover, $T\left\llcorner\mathbf{C}_{1 / 17}\right.$ is represented by the graph of $g$ with appropriate orientation and multiplicity.

For this we recall the definition of the good points $y$ in the proof of the Lipschitz approximation Lemma 3.1 (see (3.7)): $y \in \mathbf{U}_{\varrho}$ (and $y_{n} \neq 0$ in the boundary case) with $\mathbf{E}(T, y, r) \leq \eta$ for $0<r<1-\varrho$, where $\eta^{-1}=\operatorname{const}(n, \Lambda)$ (since we may assume $\omega \leq 1$ 
here). Comparing with (6.3) we see that the bad set is empty, if $\epsilon$ is chosen sufficiently small. This means that $\mathbf{C}_{\varrho} \cap$ spt $T$ in the interior case and $\mathbf{C}_{+} \cap \mathbf{C}_{\varrho} \cap \operatorname{spt} T$ in the boundary case equals graph $g$, where $g$ denotes here restriction of the Lipschitz approximation to $\mathbf{U}_{\varrho}$ or to $\mathbf{U}_{+} \cap \mathbf{U}_{\varrho}$ respectively. According to Lemma 3.1 we have $\operatorname{Lip} g \leq 1, g(0)=0$ in the interior case, $g$ has zero boundary values on $\mathbf{U}_{\varrho}^{n-1} \times\{0\}$ in the boundary case, and we may take $\varrho=\frac{1}{17}$. For the current $T_{g}$ representing graph $g$ with appropriate orientation and multiplicity we have $\mathbf{p}_{\#} T_{g}=\left(\mathbf{p}_{\#} T\right)\left\llcorner\mathbf{U}_{\varrho}\right.$. Then $T\left\llcorner\mathbf{C}_{\varrho}=T_{g}\right.$ or $T\left\llcorner\left(\mathbf{C}_{+} \cap \mathbf{C}_{\varrho}\right)=T_{g}\right.$ follows, because $y \mapsto(y, g(y))$ is biLipschitz.

In the boundary case $T \in \mathcal{T}_{b}(n, k, 1)$ we must also prove $\left(\mathbf{C}_{\varrho} \backslash \mathbf{C}_{+}\right) \cap \operatorname{spt} T \subset\left(\mathbf{U}^{n-1} \times\right.$ $\{0\}) \times\{0\}^{k}$. Suppose $x=(y, z) \in \mathbf{C}_{\varrho} \cap \operatorname{spt} T$ such that $y_{n}<0$ or $y_{n}=0$ and $z \neq 0$. Choose $r=\left|y_{n}\right|$ if $y_{n}<0$ or $r=\frac{1}{\sqrt{2}}|z|$ else. On account of $\operatorname{Lip} g \leq 1$ we then have $\mathbf{U}(x, r) \cap$ graph $g=\emptyset$ and $\mathbf{E}\left(T, y^{\prime}, 2 r\right) \geq 2^{-n} \mathbf{E}(T, y, r) \geq(2 r)^{-n} \mathbf{M}(T\llcorner\mathbf{U}(x, r))$, because $\mathbf{p}_{\#} T_{g}=\mathbf{p}_{\#} T$ and hence $\mathbf{p}_{\#}(T\llcorner\mathbf{U}(x, r))=0$. But from Lemma 2.1 we have a lower bound $\widetilde{C}_{1} \mathbf{M}\left(T\llcorner\mathbf{U}(x, r)) \geq r^{n}\right.$ with $\widetilde{C}_{1}=\operatorname{const}(n, \Lambda)$, hence $\mathbf{E}\left(T, y^{\prime}, 2 r\right) \geq 2^{-n} \widetilde{C}_{1}^{-1}$ follows. On the other hand, by choosing $\epsilon$ small enough we can achieve $\mathbf{E}\left(T, y^{\prime}, 2 r\right)<2^{-n} \widetilde{C}_{1}^{-1}$ in step 1 , and with this contradiction step 2 is finished.

For the next step in the regularity proof we need

6.2 Lemma. Suppose $g, h: \mathbb{R}^{n} \rightarrow \mathbb{R}^{k}$ are Lipschitz mappings and differentiable at $y \in \mathbb{R}^{n}$. Let $\vec{G}(y), \vec{H}(y)$ be the orientation vectors of graph $g$ and graph $h$. Then

$$
|D g(y)-D h(y)|^{2} \leq 4\left[1+(\operatorname{Lip} g)^{2}\right]^{n}\left[1+(\operatorname{Lip} h)^{2}\right]^{n}|\vec{G}(y)-\vec{H}(y)|^{2} .
$$

Proof. We have $\vec{G}(y)=\frac{\xi}{|\xi|}$ for $\xi=\overrightarrow{\mathbf{e}}+\sum_{i=1}^{n} \sum_{j=1}^{k} \xi_{i}^{j} \overrightarrow{\mathbf{e}}_{j}^{i}+\vec{r}$, where $\xi_{i}^{j}=\partial_{i} g^{j}(y)$, $\overrightarrow{\mathbf{e}}_{j}^{i}=e_{1} \wedge \ldots \wedge e_{i-1} \wedge e_{n+j} \wedge e_{i+1} \wedge \ldots \wedge e_{n}$ in terms of the canonical basis $e_{1}, \ldots, e_{n+k}$ of $\mathbb{R}^{n+k}$, and $\vec{r} \in \bigwedge_{n} \mathbb{R}^{n+k}$ is orthogonal to $\overrightarrow{\mathbf{e}}$ and all the $\overrightarrow{\mathbf{e}}_{j}^{i}$. Similarly $\vec{H}(y)=\frac{\zeta}{|\zeta|}$ with $\zeta=\overrightarrow{\mathbf{e}}+$ $\sum_{i=1}^{n} \sum_{j=1}^{k} \zeta_{i}^{j} \overrightarrow{\mathbf{e}}_{j}^{i}+\vec{s}$. The clain then follows from

$$
|\xi-\zeta|^{2} \geq\left|\left(\xi_{j}^{i}\right)-\left(\zeta_{j}^{i}\right)\right|^{2}=|D g(y)-D h(y)|^{2}
$$

and

$$
\begin{aligned}
|\xi-\zeta| & \leq|\xi|\left|\frac{\xi}{|\xi|}-\frac{\zeta}{|\zeta|}\right|+|\xi||\zeta|\left|\frac{1}{|\xi|}-\frac{1}{|\zeta|}\right| \\
& \leq|\xi|(1+|\zeta|)\left|\frac{\xi}{|\xi|}-\frac{\zeta}{|\zeta|}\right| \leq 2|\xi||\zeta|\left|\frac{\xi}{|\xi|}-\frac{\zeta}{|\zeta|}\right|
\end{aligned}
$$

where we have used $\xi \cdot \overrightarrow{\mathbf{e}}=1=\zeta \cdot \overrightarrow{\mathbf{e}},|\zeta| \geq 1$, and $|\xi|^{2} \leq\left[1+(\operatorname{Lip} g)^{2}\right]^{n}$ resp. $|\zeta|^{2} \leq$ $\left[1+(\operatorname{Lip} h)^{2}\right]^{n}$ in the last inequality.

We continue with the proof of the $\epsilon$-regularity-theorem:

Step 3 (Interior regularity). Here we treat the case $T \in \mathcal{T}_{i}(n, k, m)$ and define $D^{*} g(y)$ as the Lebesgue value of $D g$ at $y \in \mathbf{U}_{\varrho}$ whenever it exists. We consider $a, b \in \mathbf{U}_{\varrho}$ (with $\varrho=\frac{1}{17}$ ) and let $T_{a}=\boldsymbol{\tau}_{(a, g(a)) \#}^{-1} T$. By (6.3) we can apply Lemma 5.4 to $\boldsymbol{\mu}_{r_{0} \#} T_{a}\llcorner\mathbf{C}$, where $0<r_{0} \leq 1-|a|$, and we obtain a linear isometry, ${ }_{a}$ of $\mathbb{R}^{n+k}$ such that, by (5.40),

$$
\mathbf{E}\left(,{ }_{a \#} T_{a}, 0, r\right) \leq C_{26}\left[\left(\frac{r}{r_{0}}\right)^{2 \beta} \mathbf{E}\left(T, a, r_{0}\right)+\widehat{\mathrm{K}}(r)+\Omega(r)\right]
$$


for $0<r \leq \frac{1}{3} r_{0}$. (Note that we can write, ${ }_{a \#} T_{a}$ instead of , $a \#\left(T_{a}\left\llcorner\mathbf{C}_{r_{0} / 2}\right)\right.$ in (6.5), because $\left\|,{ }_{a}-\mathrm{id}\right\|$ is small and $T_{a}$ has height $\leq r_{0}$ in $\mathbf{C}_{r_{0}}$, cf. the end of step 1.) Representing , ${ }_{a}^{-1}\left(\mathbb{R}^{n} \times\{0\}^{k}\right)$ as the graph of a linear function $L_{a} \in \operatorname{Hom}\left(\mathbb{R}^{n}, \mathbb{R}^{k}\right)$, recalling Lip $g \leq 1$, and observing $\left\|L_{a}\right\| \leq 1$ on account of (5.39) and (6.1) (with $\epsilon$ small enough), we deduce from Lemma 6.2 , for $0<r \leq r_{0}$,

$$
\begin{aligned}
& \int_{\mathbf{U}_{r / 2}(a) \cap \mathbf{U}_{\varrho}}\left|D g(y)-L_{a}\right|^{2} d \mathcal{L}^{n} y \leq 2^{2 n+2} \int_{\mathbf{U}_{r / 2}(a) \cap \mathbf{U}_{\varrho}}\left|\vec{T}(y, g(y))-\left(\bigwedge_{n},{ }_{a}^{-1}\right) \overrightarrow{\mathbf{e}}\right|^{2} d \mathcal{L}^{n} y \\
& \quad \leq 2^{2 n+2} \int_{\mathbf{C}_{r / 2}(a)}\left|\vec{T}(x)-\left(\bigwedge_{n},{ }_{a}^{-1}\right) \overrightarrow{\mathbf{e}}\right|^{2} d\|T\| x \leq 2^{2 n+2} \int_{\mathbf{C}_{r}}\left|\overrightarrow{{ }_{a \#} T_{a}}-\overrightarrow{\mathbf{e}}\right|^{2} d\left\|,{ }_{a \#} T_{a}\right\| x \\
& \quad=2^{2 n+3} r^{n} \mathbf{E}\left(,{ }_{a \#} T_{a}, 0, r\right) .
\end{aligned}
$$

(We have used here, ${ }_{a}\left(\mathbf{C}_{r / 2} \cap \operatorname{spt} T_{a}\right) \subset \mathbf{C}_{r}$ which is true, because $T_{a}$ has height $\leq r$ in $\mathbf{C}_{r}$ and $\left\|,{ }_{a}-\mathrm{id}\right\|$ is small.) Letting $r \downarrow 0$ we conclude with $(6.5)$ that $D^{*} g(a)=L_{a}$ and

$$
\left(\bigwedge_{n},{ }_{a}^{-1}\right) \overrightarrow{\mathbf{e}}=\vec{T}^{*}(a, g(a)),
$$

the Lebesgue value of $\vec{T}$ at $(a, g(a))$ with respect to the measure $\|T\|=m \mathcal{H}^{n} L$ graph $g$.

From (6.5), (6.6) and the analogous inequality for $L_{b}=D^{*} g(b)$ with $r=2|b-a| \leq \frac{4}{17}$ we also deduce

$$
\begin{aligned}
& \left|D^{*} g(b)-D^{*} g(a)\right|^{2} \leq \frac{2^{n+1}}{\boldsymbol{\alpha}(n)|b-a|^{n}} \int_{\mathbf{U}_{r / 2}(a) \cap \mathbf{U}_{r / 2}(b) \cap \mathbf{U}_{\varrho}}\left|L_{a}-L_{b}\right|^{2} d \mathcal{L}^{n} \\
& \leq \frac{2^{2 n+2}}{\boldsymbol{\alpha}(n) r^{n}}\left[\int_{\mathbf{U}_{r / 2}(a) \cap \mathbf{U}_{\varrho}}\left|D g(y)-L_{a}\right|^{2} d \mathcal{L}^{n} y+\int_{\mathbf{U}_{r / 2}(b) \cap \mathbf{U}_{\varrho}}\left|D g(y)-L_{b}\right|^{2} d \mathcal{L}^{n} y\right] \\
& \leq C_{27}\left[r^{2 \beta} \mathbf{E}(T, 0,1)+\widehat{\mathbf{K}}(r)+\Omega(r)\right]
\end{aligned}
$$

with $C_{27}=\operatorname{const}(n, k, m, \lambda, \Lambda, \nu(\cdot), \beta)$. Since $r=2|b-a|$ and $\widehat{\mathrm{K}}(2 r)+\Omega(2 r) \leq 2^{2 \alpha}(\widehat{\mathrm{K}}(r)+$ $\Omega(r)$ ) the continuity modulus (6.2) for $D g$ is proved.

Step 4 (boundary regularity). Here we consider $T \in \mathcal{T}_{b}(n . k, 1)$ and first distinguish several special cases for $a, b \in \mathbf{B}_{+} \cap \mathbf{U}_{\varrho}\left(\varrho=\frac{1}{17}\right)$.

The special case 1 is $a, b \in \mathbf{U}_{\varrho}^{n-1} \times\{0\}$. In this case we can proceed exactly as in Step 3 , using the boundary version of Lemma 5.4 and $\mathrm{K}(r)^{2}+\widehat{\mathrm{M}}(r) \leq 5 \widehat{\mathrm{K}}(r)$. The result is that the Lebesgue values of $D g$ at $a, b$ exist (defined here with half balls $\mathbf{U}_{+} \cap \mathbf{U}_{r}(a)$, of course, instead of balls $\left.\mathbf{U}_{r}(a)\right)$ and satisfy

$$
\left|D^{*} g(b)-D^{*} g(a)\right|^{2} \leq C_{27}\left[|b-a|^{2 \beta} \mathbf{E}(T, 1)+\widehat{\mathrm{K}}(|b-a|)+\Omega(|b-a|)\right],
$$

if we redefine $C_{27}$ suitably.

The special case 2 is $a \in \mathbf{U}_{\varrho}^{n-1} \times\{0\}$ and $b \in \mathbf{U}_{\varrho}$ with $b_{n}>0$ and $|a-b| \leq 2 b_{n}$. We define $T_{a},{ }_{a}$ as in step 3 and have (6.5) again for $r_{0} \leq 1-|a|$ and $0<r<\frac{1}{3} r_{0}$. We next want to apply the interior regularity result from step 3 to , a\# $T$ in $\mathbf{C}_{r_{1}}(\widehat{b})$ where 
${ }_{a}(b, g(b))=(\widehat{b}, \widehat{c})$ and $r_{1}=\widehat{b}_{n}$. Recalling that , $a=\mathrm{id}$ and $g=0$ on $\mathbf{U}_{\varrho}^{n-1} \times\{0\}$, and $\operatorname{Lip} g \leq 1$, we see

$$
\left|b_{n}-r_{1}\right|=|b-\widehat{b}| \leq\left|(b, g(b))-{ }_{a}(b, g(b))\right| \leq \sqrt{2}\left\|,{ }_{a}-\mathrm{id}\right\| b_{n} .
$$

Since $\left\|,{ }_{a}-\mathrm{id}\right\|$ is small we conclude $\frac{4}{5} b_{n} \leq r_{1} \leq \frac{6}{5} b_{n}$ and $\mathbf{U}_{r_{1}}(\widehat{b}) \subset \mathbf{U}_{+} \cap \mathbf{U}_{4 b_{n}}(a)$, hence

$$
\begin{aligned}
\mathbf{E}\left(,{ }_{a \#} T, \widehat{b}, r_{1}\right) & \leq 5^{n} \mathbf{E}\left(,{ }_{a \#} T, a, 4 b_{n}\right) \\
& \leq 5^{n} C_{26}\left[\left(\frac{17}{4} b_{n}\right)^{2 \beta} \mathbf{E}\left(T, a, \frac{16}{17}\right)+\widehat{\mathrm{K}}\left(4 b_{n}\right)+\Omega\left(4 b_{n}\right)\right],
\end{aligned}
$$

by (6.5) applied with $r_{0}=\frac{16}{17}$ and $r=4 b_{n} \leq \frac{4}{17}$. (From (6.3) we could not obtain a bound on $\mathbf{E}\left(, a_{a \#} T, \widehat{b}, r_{1}\right)$ which is small for small $b_{n}>0$ as in $(6.10)$. This is the reason why we must apply the interior excess growth lemma to , ${ }_{a \#} T$ instead of $T$.)

Now, as in case 1 and step 3 the Lebesgue values $D^{*} g(a), D^{*} g(b)$ exist with

$$
\begin{aligned}
& r^{-n} \int_{\mathbf{U}_{\varrho}^{+} \cap \mathbf{U}_{r / 2}(a)}\left|D g(y)-D^{*} g(a)\right|^{2} d \mathcal{L}^{n} y \\
& \leq 2^{2 n+3} C_{26}\left[\left(\frac{r}{r_{0}}\right)^{2 \beta} \mathbf{E}\left(T, a, r_{0}\right)+\widehat{\mathrm{K}}(r)+\Omega(r)\right]
\end{aligned}
$$

for $0<r \leq \frac{1}{3} r_{0} \leq \frac{1}{3}(1-|a|)$, by the analogs of (6.5) and (6.6), and

$$
\begin{aligned}
& \int_{\mathbf{U}_{r / 3}(b) \cap \mathbf{U}_{\varrho}}\left|D g(y)-D^{*} g(b)\right|^{2} d \mathcal{L}^{n} y \\
& \leq 2^{2 n+2} \int_{\mathbf{C}_{r / 3}(b)}\left|\vec{T}(x)-\vec{T}^{*}(b, g(b))\right|^{2} d\|T\| x \\
& \leq 2^{2 n+2} \int_{\mathbf{C}_{r / 2}(\widehat{b})}\left|\overrightarrow{{ }_{a \#}} \vec{T}(x)-{\overrightarrow{{ }_{a \#}}}^{*}(\widehat{b}, \widehat{c})\right|^{2} d\left\|,{ }_{a \#} T\right\| x
\end{aligned}
$$

for $0<r \leq b_{n}$, by the analogs of (6.6), (6.7) at $b$ and the fact that $\left\|,{ }_{a}-\mathrm{id}\right\|$ is small, spt $T$ is the graph a function vanishing on $\mathbf{U}_{\varrho}^{n-1} \times\{0\}$ with Lipschitz constant $\leq 1$, and ${ }_{, a}\left(\mathbf{C}_{r / 3}(b) \cap \operatorname{spt} T\right) \subset \mathbf{C}_{r / 2}(\widehat{b}) \cap,{ }_{a} \operatorname{spt} T$.

Decreasing $\epsilon$ if necessary we see from (6.10) that, ${ }_{a \#} T$, after translating $(\widehat{b}, \widehat{c})$ to the origin and applying the homothety $\boldsymbol{\mu}_{r_{1}}^{-1}$, satisfies the hypotheses of Lemma 5.4. The analogs of (6.6), (6.7), (6.5) for , ${ }_{a \#} T, \widehat{b}, r \leq r_{1}$ instead of $T, a, r \leq r_{0}$ then give

$$
\begin{aligned}
2^{2 n+2} \int_{\mathbf{C}_{r / 2}(\widehat{b})}\left|\overrightarrow{{ }_{a \#}} \vec{T}(x)-\overrightarrow{, a \#}^{*}(\widehat{b}, \widehat{c})\right|^{2} d\left\|,{ }_{a \#} T\right\| x \\
\leq C_{28} r^{n}\left[\left(\frac{r}{r_{1}}\right)^{2 \beta} \mathbf{E}\left(,{ }_{a \#} T, \widehat{b}, r_{1}\right)+\widehat{\mathrm{K}}(r)+\Omega(r)\right],
\end{aligned}
$$

with $C_{28}=\operatorname{const}(n, k, m . \lambda, \Lambda, \beta)$. Combinig this with $(6.12)$ we have, for $0<r \leq \frac{1}{3} r_{1}$,

$$
\begin{aligned}
r^{-n} \int_{\mathbf{U}_{r / 3}(b) \cap \mathbf{U}_{\varrho}}\left|D g(y)-D^{*} g(b)\right|^{2} d \mathcal{L}^{n} y \\
\leq C_{28}\left[\left(\frac{r}{r_{1}}\right)^{2 \beta} \mathbf{E}\left(,{ }_{a \#} T, \widehat{b}, r_{1}\right)+\widehat{\mathrm{K}}(r)+\Omega(r)\right] .
\end{aligned}
$$


We now choose $r=\frac{1}{3} r_{1}$ in (6.14), $r=2|b-a|$ and $r_{0}=\frac{16}{17}$ in (6.11) and recall $\frac{2}{5}|b-a| \leq \frac{4}{5} b_{n} \leq r_{1}=\widehat{b}_{n} \leq \frac{6}{5} b_{n} \leq \frac{6}{5}|b-a|, \Omega(t \varrho) \leq t^{2 \alpha} \Omega(\varrho)$ and $\widehat{\mathrm{K}}(t \varrho) \leq t^{2 \alpha} \widehat{\mathrm{K}}(\varrho)$ for $t \geq 1$, to deduce

$$
\begin{gathered}
\int_{\mathbf{U}_{r_{1} / 9}(b) \cap \mathbf{U}_{\varrho}}\left|D g(y)-D^{*} g(b)\right|^{2} d \mathcal{L}^{n} y+\int_{\mathbf{U}_{\varrho}^{+} \cap \mathbf{U}_{|b-a|}(a)}\left|D g(y)-D^{*} g(a)\right|^{2} d \mathcal{L}^{n} y \\
\leq|b-a|^{n} C_{29}\left[|b-a|^{2 \beta} \mathbf{E}(T, 0,1)+\widehat{\mathbf{K}}(|b-a|)+\Omega(|b-a|)\right],
\end{gathered}
$$

where $C_{29}=\operatorname{const}(n, k, m, \lambda, \Lambda, \beta)$. Since $\mathbf{U}_{r_{1} / 9}(b) \cap \mathbf{U}_{\varrho}^{+} \cap \mathbf{U}_{|b-a|}(a)$ contains a half-ball of radius $\frac{1}{18} r_{1} \geq \frac{1}{45}|b-a|$ we conclude as in (6.8)

$$
\left|D^{*} g(b)-D^{*} g(a)\right|^{2} \leq C_{30}\left[|b-a|^{2 \beta} \mathbf{E}(T, 0,1)+\widehat{\mathrm{K}}(|b-a|)+\Omega(|b-a|)\right]
$$

with $C_{30}=\operatorname{const}(n, k, m, \lambda, \Lambda, \beta)$.

The special case 3 is $b, \tilde{b} \in \mathbf{U}_{\varrho}$ with $0<b_{n} \leq \tilde{b}_{n}$ and $|b-\tilde{b}| \leq \frac{2}{15} b_{n}$. In this case we choose $a \in \mathbf{U}_{\varrho}^{n-1} \times\{0\}$ such that $|a-b| \leq 2 b_{n}$ and $|a-\tilde{b}| \leq 2 \tilde{b}_{n}$. From (6.14) with $r=2|b-\tilde{b}|$ and from (6.10) we obtain, recalling $r_{1} \geq \frac{4}{5} b_{n}$,

$$
\begin{array}{r}
|b-\tilde{b}|^{-n} \int_{\mathbf{U}_{2|b-\tilde{b}| / 3}(b) \cap \mathbf{U}_{e}}\left|D g(y)-D^{*} g(b)\right|^{2} d \mathcal{L}^{n} y \leq C_{31}\left[|b-\tilde{b}|^{2 \beta} \mathbf{E}(T, 0,1)\right. \\
\left.\quad+\widehat{\mathrm{K}}(2|b-\tilde{b}|)+\Omega(2|b-\tilde{b}|)+\left|\frac{b-\tilde{b}}{b_{n}}\right|^{2 \beta} \widehat{\mathrm{K}}\left(4 b_{n}\right)+\left|\frac{b-\tilde{b}}{b_{n}}\right|^{2 \beta} \Omega\left(4 b_{n}\right)\right]
\end{array}
$$

with $C_{31}=\operatorname{const}(n, k, m, \lambda, \Lambda, \beta)$, and the same estimate is valid with $b, \tilde{b}$ interchanged. On account of $\beta \geq \alpha$ the exponent $2 \beta$ can be replaced by $2 \alpha$ in the last two summands, and from $\widehat{\mathrm{K}}(t \varrho)+\Omega(t \varrho) \leq t^{2 \alpha}(\widehat{\mathrm{K}}(\varrho)+\Omega(\varrho))$ for $t \geq 1$ and the fact that $\mathbf{U}_{2|b-\tilde{b}| / 3}(b) \cap \mathbf{U}_{2|b-\tilde{b}| / 3}(\tilde{b}) \cap \mathbf{U}_{\varrho}$ contains a half-ball of radius $\frac{1}{6}|b-\tilde{b}|$ we infer

$$
\left|D^{*} g(b)-D^{*} g(\tilde{b})\right|^{2} \leq C_{32}\left[|b-\tilde{b}|^{2 \beta} \mathbf{E}(T, 0,1)+\widehat{\mathrm{K}}(|b-\tilde{b}|)+\Omega(|b-\tilde{b}|)\right]
$$

with $C_{32}=\operatorname{const}(n, k, m, \lambda, \Lambda, \beta)$.

In the general case we consider $a, b \in \mathbf{B}_{+} \cap \mathbf{U}_{\varrho}\left(\varrho=\frac{1}{17}\right)$ with the aim to prove the estimate (6.2). In the special cases this estimate follows from (6.9), (6.15), and (6.16). If $a \in \mathbf{U}_{\varrho}^{n-1} \times\{0\}$ and $b \in \mathbf{U}_{+} \cap \mathbf{U}_{\varrho}$ with $|b-a|>2 b_{n}$, we apply the special case 1 to $a$ and $b^{\prime}=\left(b_{1}, \ldots, b_{n-1}, 0\right)$ and the special case 2 to $b^{\prime}$ and $b$, and (6.2) follows because $\left|a-b^{\prime}\right| \leq|a-b|$ and $\left|b-b^{\prime}\right| \leq|a-b|$. The only remaining case is $a, b \in \mathbf{U}_{\varrho}$ with $0<a_{n} \leq b_{n}$ and $|a-b|>\frac{2}{15} a_{n}$. In this case we apply the special case 1 to $a^{\prime}=\left(a_{1}, \ldots, a_{n-1}, 0\right)$ and $b^{\prime}=$ $\left(b_{1}, \ldots, b_{n-1}, 0\right)$, and the special case 2 to $a^{\prime}, a$ and to $b^{\prime}, b$. On account of $\left|a^{\prime}-b^{\prime}\right| \leq|a-b|$, $\left|a-a^{\prime}\right|=a_{n}<\frac{15}{2}|a-b|$, and $\left|b-b^{\prime}\right| \leq|b-a|+\left|a-a^{\prime}\right|+\left|a^{\prime}-b^{\prime}\right| \leq \frac{19}{2}|a-b|$ the estimate (6.2) then follows again from (6.9) and (6.15), since $\widehat{\mathrm{K}}(t \varrho)+\Omega(t \varrho) \leq t^{2 \alpha}(\widehat{\mathrm{K}}(\varrho)+\Omega(\varrho))$ for $t \geq 1$.

The preceding theorem is not completely satisfactory with regard to boundary regularity, for the following reason: if $T$ is $(F, \omega)$ minimizing for a smooth integrand $F$ and $\partial T$ is 
represented locally by an $(n-1)$-dimensional oriented submanifold $B$ of $\mathbb{R}^{n+k}$ with multiplicity 1 and with continuity modulus $\kappa$ for the tangent plane field of $B$, then we can flatten the boundary locally by transforming $B$ into $\left(\mathbf{U}^{n-1} \times\{0\}\right) \times\{0\}^{k}$ with a diffeomorphism $\Phi^{-1}$ of class $C^{1, \kappa(\cdot)}$ and apply Theorem 6.1 to the transformed curent $\tilde{T}=\Phi_{\#}^{-1} T$ and to the transformed integrand $\tilde{F}=\Phi_{\#} F$ (provided the hypotheses of the theorem are satisfied by $\tilde{T}$ and $\tilde{F})$. However, the continuity modulus of $\tilde{F}$ and $D_{(2)} \tilde{F}$ with respect to the space variable will not be better than $\kappa$, in general, hence Theorem 6.1 will only give $C^{1}$ regularity for $T$ with the modulus $(\widehat{\mathrm{K}}+\Omega)^{1 / 2}$. For example, if $\kappa(r)=\operatorname{const} r^{\beta}$ with $0<\beta<1$ and $\omega(r)=$ const $r^{2 \beta}$ then we obtain only the modulus const $r^{\beta /(2-\beta)}$ for the tangent plane field of $T$, wheras the expected boundary regularity of $T$ in this situation is $C^{1, \beta}$. Using the full strength of Lemma 5.4, however, we can prove the continuity modulus $\left(\mathrm{K}^{2}+\Omega\right)^{1 / 2}$ for the tangent plane field of $T$, i.e. the optimal $C^{1, \beta}$ boundary regularity in the example. For this we need the following variant of Lemma 5.3 for nonlinear mappings:

6.3 Lemma (excess comparison for diffeomorhisms). Suppose $\Phi$ is a $C^{1}$ diffeomorphism between open sets of $\mathbb{R}^{n+k}$, $\sup \|D \Phi\| \leq L$ and $\sup \left\|D \Phi^{-1}\right\| \leq L$ with $1 \leq L<\infty$, and $T \in \mathcal{R}_{n}\left(\mathbb{R}^{n+k}\right)$ such that $\mathbf{p}_{\#}\left(T\left\llcorner\mathbf{C}_{r}(y)\right)\right.$ and $\mathbf{p}_{\#}\left(\left(\Phi_{\#} T\right)\left\llcorner\mathbf{C}_{\tilde{r}}(\tilde{y})\right)\right.$ have nonnegative multiplicities and $\mathbf{C}_{\tilde{r}}(\tilde{y}) \cap \operatorname{spt}\left(\Phi_{\#} T\right)$ is contained in $\Phi\left(\mathbf{C}_{r}(y) \cap \operatorname{spt} T\right)$. Then we have

$$
\tilde{r}^{n} \mathbf{E}\left(\Phi_{\#} T, \tilde{y}, \tilde{r}\right) \leq 2 L^{n} r^{n} \mathbf{E}(T, y, r)+n^{2} L^{3 n-2} \mathbf{M}\left(T\left\llcorner\mathbf{C}_{r}(y)\right) \sup _{\mathbf{C}_{r}(y) \cap \operatorname{spt} T}\|D \Phi-\mathrm{id}\|^{2} .\right.
$$

Proof. From the area formula [Fe, 3.2.20, 4.1.30] and (1.24) we obtain

$$
\begin{aligned}
2 \tilde{r}^{n} \mathbf{E}\left(\Phi_{\#} T, \tilde{y}, \tilde{r}\right) & =\int_{\mathbf{C}_{\tilde{r}}(\tilde{y})}\left|\overrightarrow{\Phi_{\#} \vec{T}}-\overrightarrow{\mathbf{e}}\right|^{2} d\left\|\Phi_{\#} T\right\| \\
& \leq \int_{\mathbf{C}_{r}(y)}\left|\overrightarrow{\Phi_{\#}} \vec{T}(\Phi(x))-\overrightarrow{\mathbf{e}}\right|^{2}\left|\left(\bigwedge_{n} D \Phi(x)\right) \vec{T}(x)\right| d\|T\| x \\
& \leq 2 L^{n} \int_{\mathbf{C}_{r}(y)}\left[|\vec{T}-\overrightarrow{\mathbf{e}}|^{2}+\left|\left(\bigwedge_{n} D \Phi\right) \vec{T}-\right|\left(\bigwedge_{n} D \Phi\right) \vec{T}|\vec{T}|^{2}\right] d\|T\|,
\end{aligned}
$$

because $L^{-n} \leq\left|\left(\bigwedge_{n} D \Phi\right) \vec{T}\right| \leq L^{n}$ and $\overrightarrow{\Phi_{\#} T}(\Phi(x))=\left|\left(\bigwedge_{n} D \Phi(x)\right) \vec{T}(x)\right|^{-1}\left(\bigwedge_{n} D \Phi(x)\right) \vec{T}(x)$. The claim then follows with

$$
\left|\left(\bigwedge_{n} D \Phi(x)\right) \vec{T}(x)-\vec{T}(x)\right| \leq\|D \Phi-\mathrm{id}\| \sum_{j=0}^{n-1}\|D \Phi(x)\|^{j} .
$$

It is clear that a variant of the Lemma holds for $\Phi$ only biLipschitz, where $D \Phi$ above has to be interpreted as the approximative differential with respect to the rectifiable set underlying $T$.

For the formulation of the optimal small excess boundary regularity theorem we now fix some notations and assumptions: We consider $T=T\left\llcorner\mathbf{C} \in \mathcal{R}_{n}\left(\mathbb{R}^{n+k}\right)\right.$ such that $\partial T$ is represented in $\mathbf{C}$ by the graph of a $C^{1}$ function $\left(\varphi_{0}, \ldots, \varphi_{k}\right)=\varphi: \mathbf{U}^{n-1} \rightarrow \mathbb{R}^{1+k}$, taken with suitable orientation and multiplicity 1 . We assume $\varphi(0)=0, D \varphi(0)=0$, and

$$
\left\|D \varphi\left(y^{\prime}\right)-D \varphi\left(y^{\prime \prime}\right)\right\| \leq \kappa\left(\left|y^{\prime}-y^{\prime \prime}\right|\right) \quad \text { for } y^{\prime}, y^{\prime \prime} \in \mathbf{U}^{n-1}
$$


where $\kappa$ is a continuity modulus for the tangent plane field of the boundary $(\partial T)\llcorner\mathbf{C}$. We assume $\kappa$ sufficiently small so that $\mathbf{U}$ is divided into two components by graph $\varphi_{0}=$ $\mathbf{p}(\operatorname{graph} \varphi)$, and for $\mathbf{p}_{\#} T$ we assume that it has multiplicities 1 on $D_{+}=\left\{\left(y^{\prime}, y_{n}\right) \in \mathbf{U}\right.$ : $\left.y_{n}>\varphi_{0}\left(y^{\prime}\right)\right\}$ and multiplicity 0 on $D_{-}=\left\{\left(y^{\prime}, y_{n}\right) \in \mathbf{U}: y_{n}<\varphi_{0}\left(y^{\prime}\right)\right\}$, i.e. $\mathbf{p}_{\#} T=\mathbf{E}^{n}\left\llcorner D_{+}\right.$. For the integrand $F$ we require all conditions from Section 1 in particular the bound $\Lambda$, the ellipticity constant $\lambda$, and the modulus $\nu$ for $\xi \mapsto D_{(2)}^{2} F(x, \xi)$ from (1.4), but we denote the continuity modulus for $x \mapsto F(x, \xi)$ and $x \mapsto D_{2} F(x, \xi)$ by $\mu$ this time, i.e. instead of (1.5), (1.6) we have

$$
\begin{gathered}
|F(x, \xi)-F(\tilde{x}, \xi)| \leq \mu(|x-\tilde{x}|)|\xi|, \\
\left\|D_{(2)} F(x, \xi)-D_{(2)} F(\tilde{x}, \xi)\right\| \leq \mu(|x-\tilde{x}|),
\end{gathered}
$$

and we do not make use of the special modulus introduced in (1.7), (1.8) (and denoted $\mu$ there). For the functions $\kappa, \mu, \omega$ we assume all the conditions stated in Section 1. In particular there exists $0<\alpha<1$ such that $r^{-\alpha} \kappa(r), r^{-\alpha} \mu(r)$ and $r^{-\alpha} \sqrt{\omega(r)}$ are nonincreasing functions of $r>0$. With $\mu$ we associate the function $\widehat{\mu}$ by (1.9) and with $\kappa, \widehat{\mu}, \omega$ the functions $\mathrm{K}, \widehat{\mathrm{M}}, \Omega$ by (1.15). We then have

6.4 Theorem (optimal boundary $\epsilon$-regularity theorem). For $\alpha \leq \beta<1$ there exists a positive constant $\epsilon_{1}(n, k, \lambda, \Lambda, \nu(\cdot), \beta)$ sucht that if $T, F$ satify the assumptions above, $T$ is $(F, \omega)$ minimizing in $\mathbf{C}$ and

$$
\mathbf{E}(T, 1)+\mathrm{K}(1)^{2}+\widehat{\mathrm{M}}(1)+\Omega(1) \leq \epsilon_{1},
$$

then $T\left\llcorner\mathrm{C}_{1 / 50}\right.$ is represented by the graph, taken with multiplicity 1 , of a continuously differentiable function $g: \mathbf{U}_{1 / 50} \cap \mathbf{p}(\operatorname{spt} T) \rightarrow \mathbb{R}^{k}$. Moreover, the derivative $D g$ has the following modulus of continuity:

$$
|D g(a)-D g(b)| \leq C_{41}\left[r^{2 \beta} \mathbf{E}(T, 1)+\mathrm{K}(r)^{2}+\widehat{\mathrm{M}}(r)+\Omega(r)\right]^{1 / 2}
$$

for $a, b \in \mathbf{U}_{1 / 50} \cap \mathbf{p}(\operatorname{spt} T)$ with $|a-b| \leq r$, where $C_{41}=\operatorname{const}(n, k, \lambda, \Lambda, \beta)$.

For example, if the boundary is of class $C^{1, \beta}$, i.e. $\kappa(r) \leq \operatorname{const} r^{\beta}$ for small $r>0$, if the integrand is smooth $\left(\mu(r) \leq\right.$ const $r^{2 \beta /(1+\beta)}$ is sufficient, as then $\widehat{\mathrm{M}}(r) \leq$ const $r^{2 \beta}$ follows $)$, and if $\omega(r) \leq$ const $r^{2 \beta}$, then (6.18) gives Hölder continuity of $D g$ up to the boundary with exponent $\beta \in] 0,1[$. This is, of course, the best possible boundary regularity result, if the prescribed boundary is merely of class $C^{1, \beta}$. If the boundary is of Dini class $C^{1, \kappa(\cdot)}$, where $r \mapsto r^{-\beta} \kappa(r)>0$ is decreasing for all $0<\beta<1$, then Theorem gives $C^{1, \mathrm{~K}(\cdot)}$ Dini boundary regularity, provided $\mathrm{K}(1)=\int_{0}^{1} \frac{1}{\varrho} \kappa(\varrho) d \varrho$ is finite and $\widehat{\mathrm{M}}(r), \Omega(r)$ are dominated by $\mathrm{K}(r)$ as $r \downarrow 0$ (e.g. $\mu(r) \leq$ const $r^{\beta}, \omega(r) \leq$ const $r^{2 \beta}$ as above, for some $0<\beta<1$ ).

Proof. We define $\Phi(\tilde{x})=\tilde{x}+\left(0, \varphi\left(\tilde{x}_{1}, \ldots, \tilde{x}_{n-1}\right)\right) \in \mathbb{R}^{n-1} \times \mathbb{R}^{1+k}$ for $\tilde{x} \in \mathbf{C}$ so that $\Phi^{-1}(x)=x-\left(0, \varphi\left(x_{1}, \ldots, x_{n-1}\right)\right)$. If $\epsilon_{1}$, and hence also $\kappa(1) \leq \mathrm{K}(1)$, is sufficiently small, we have $\operatorname{Lip} \varphi \leq \kappa(1) \leq \frac{1}{7}$ and $\operatorname{Lip} \Phi \leq \frac{8}{7}, \operatorname{Lip} \Phi^{-1} \leq \frac{8}{7}$. Then $\Phi\left(\mathbf{C}_{7 / 8}\right) \subset \mathbf{C}$ follows because $\Phi(0)=0$, and $\Phi$ acts on $\{y\} \times \mathbb{R}^{n}$ as a translation. Letting $\tilde{T}=\left(\boldsymbol{\mu}_{7 / 8 \#}^{-1} \Phi_{\#}^{-1} T\right)\llcorner\mathbf{C})$ and $\tilde{F}(\tilde{x}, \tilde{\xi})=\left(\frac{8}{7}\right)^{n} F\left(\Phi\left(\frac{7}{8} \tilde{x}, \bigwedge_{n} D \Phi\left(\frac{7}{8} \tilde{x}\right) \tilde{\xi}\right)\right.$ we verify that $\tilde{T} \in \mathcal{T}_{b}(n, k, 1)$ is $(\tilde{F}, \tilde{\omega})$ minimizing in $\mathrm{C}$ with $\tilde{\omega}(r)=\omega\left((\operatorname{Lip} \Phi) \frac{7}{8} r\right) \leq \omega(r)$. The transformed integrand $\tilde{F}$ satisfies (1.1)-(1.4) and (1.12) with bound $\tilde{\Lambda} \leq C \Lambda$, modulus $\tilde{\nu}(r) \leq C[\nu(C r)+\Lambda r]$, and ellipticity constant 
$\tilde{\lambda} \geq C^{-1} \lambda$ (by[Fe, 5.1.4]), where $C=\operatorname{const}(n, k)$. For the modulus $\tilde{\kappa}$ associated with $\tilde{F}$ by (1.5), (1.6) we obtain $\tilde{\kappa}(r) \leq C\left[\mu\left((\operatorname{Lip} \Phi) \frac{7}{8} r\right)+\Lambda \kappa\left(\frac{7}{8} r\right)\right] \leq C[\mu(r)+\Lambda \kappa(r)]$, and for the modulus $\tilde{\mu}$ associated with $\tilde{F}$ by (1.7), (1.8) we find, using the special structure of $\Phi$, i.e. the independence of $D \Phi(\tilde{x})$ from $\tilde{x}_{n}, \ldots, \tilde{x}_{n+k}$, the estimate $\tilde{\mu}(r) \leq \mu\left((\operatorname{Lip} \Phi) \frac{7}{8} r\right) \leq C \mu(r)$. It follows that $\tilde{\mathrm{X}}(r)$, defined by (5.33) and (1.15), (1.9) in terms of $\tilde{\kappa}, \tilde{\mu}, \tilde{\omega}$, does not exceed $C\left[\mathrm{~K}(r)^{2}+\mathrm{M}(r)^{2}+\widehat{\mathrm{M}}(r)+\Omega(r)\right]$, where $\mathrm{K}, \mathrm{M}, \widehat{\mathrm{M}}, \Omega$ are defined in terms of $\kappa, \mu, \omega$ as in (1.15), (1.9). If $\epsilon_{1}$, and hence also $\widehat{\mu}(1) \leq \widehat{\mathrm{M}}(1)$, is small enough, then we see $\mu(r)^{2} \leq 16 \widehat{\mu}(r)$ and $\mathrm{M}(r)^{2} \leq 16 \widehat{\mathrm{M}}(r)$ for $0<r \leq 1$, recalling the discussion following (1.9). Consequently,

$$
\tilde{\mathrm{X}}(r) \leq \operatorname{const}(n, k)\left[\mathrm{K}(r)^{2}+\widehat{\mathrm{M}}(r)+\Omega(r)\right] \quad \text { for } 0<r \leq 1 .
$$

We also note that

$$
\mathbf{E}(\tilde{T}, 1) \leq \operatorname{const}(n)\left[\mathbf{E}(T, 1)+\kappa\left(\frac{7}{8}\right)^{2}\right],
$$

by Lemma 6.3. It follows from (6.17), (6.19), (6.20) and $\kappa(1) \leq \mathrm{K}(1)$ that $\tilde{T}$ satisfies the hypotheses of Lemma 5.4 , provided $\epsilon_{1}$ is chosen sufficiently small.

In the rest of the proof we will need additional smallness conditions for $\epsilon_{1}$ in various arguments, and we will tacitly assume that $\epsilon_{1}$ has been chosen to satisfy these conditions. By $C_{34}, C_{35}, \ldots$ we denote constants depending only on $n, k, \lambda, \Lambda, \beta$. As in Step 1 of the proof of Theorem 6.1 we next establish uniform smallness

$$
\mathbf{E}(\tilde{T}, y, r)+\mathrm{K}(r)^{2}+\widehat{\mathrm{M}}(r)+\Omega(r) \leq C_{34} \epsilon_{1},
$$

for $y \in \mathbf{B}_{1 / 2}$ with $y_{n} \geq 0$ and for $r>0$ with $r \leq 1-|y|$. In the case $y_{n}=0, \frac{1}{2} \leq r \leq 1-|y|$ this follows from (6.17) and (6.20) by passing to the scaled current $\left(\boldsymbol{\mu}_{r \#}^{-1} \boldsymbol{\tau}_{(y, 0)}^{-1} \tilde{T}\right)\llcorner\mathbf{C}$, and from the excess bound (5.41) together with (6.19) we obtain (6.21) also when $y_{n}=0$, $0<r<\frac{1}{2}$. In the case $y_{n}>0$ we deduce (6.21) for $\left(\frac{7}{8}\right)^{2} y_{n} \leq r \leq 1-|y|$ by applying (6.21) with center $y^{\prime}=\left(y_{1}, \ldots, y_{n-1}, 0\right)$ and radius $r^{\prime}=2 y_{n}$ when $2 y_{n} \leq 1-\left|y^{\prime}\right|$ and with center 0 and radius 1 when $2 y_{n}>1-\left|y^{\prime}\right|$ (which implies $\left.r \geq\left(\frac{7}{8}\right)^{2} y_{n}>\left(\frac{7}{8}\right)^{2} \frac{1}{4}\right)$. Using this with $r=y_{n}$ we see from Lemma 6.3 and

$$
\mathbf{M}\left(\tilde{T}\left\llcorner\mathbf{C}_{r}(y)\right) \leq r^{n}[\boldsymbol{\alpha}(n)+\mathbf{E}(\tilde{T}, y, r)]\right.
$$

that $\left(\left(\boldsymbol{\mu}_{49 r / 64}^{-1} \boldsymbol{\tau}_{x}^{-1}\right)_{\#} T\right)\left\llcorner\mathbf{C}\right.$ for $x \in \operatorname{spt} T$ with $\mathbf{p} x=\mathbf{p} \Phi\left(\frac{7}{8} y, 0\right)$ satisfies the hypotheses of Lemma 5.4 in the interior situation (with respect to the corresponding transformation of the integrand $F$ ). From the conclusion (5.41) of this lemma we get (6.21) also for $0<r<\left(\frac{7}{8}\right)^{2} y_{n}$ by using Lemma 6.3 again (and the analogue of (6.22)). (Note that we cannot apply the interior case of lemma 5.4 directly to $\tilde{T}$, because we did not assume that $\int_{0}^{1} \frac{1}{\varrho} \sqrt{\widehat{\kappa}(\varrho)} d \varrho$ is finite.) Combining (6.21) with the height estimate of Lemma 2.2 we find that the height of $\left(\boldsymbol{\mu}_{r \#}^{-1} \boldsymbol{\tau}_{\tilde{x} \#}^{-1} \tilde{T}\right)\left\llcorner\mathbf{C}\right.$ does not exceed $\frac{1}{3}$, say, if $\tilde{x} \in \operatorname{spt} \tilde{T}, \mathbf{p} \tilde{x} \in \mathbf{B}_{1 / 2} \cap \mathbf{B}_{+}$, and $0<2 r<1-|\mathbf{p} \tilde{x}|$. Applying the diffeomorphism $\Phi$ we obtain corresponding height estimates for $T$, e.g. for $0<r \leq \frac{7}{16}$ the height of $T\left\llcorner\mathbf{C}_{r}\right.$ does not exceed $\frac{2}{3}\left(\frac{8}{7}\right)^{2} r<r$.

Exactly as in Step 2 of the proof for Theorem 6.1 we see from (6.21) that $\tilde{T}\left\llcorner\mathbf{C}_{1 / 34}\right.$ is represented by the graph, taken with multiplicity 1 , of a Lipschitz function $\tilde{g}: \mathbf{U}_{1 / 34} \cap$ $\mathbf{B}_{+} \rightarrow \mathbb{R}^{k}$ with Lip $\tilde{g} \leq 1$ and $\tilde{g}=0$ on $\mathbf{U}_{1 / 34}^{n-1} \times\{0\}$. Consequently, $T$ is represented in 
$\mathbf{C}_{1 / 50}$ (since $\left(\frac{7}{8}\right)^{2} \frac{1}{34}<\frac{1}{50}$ ) by the graph of a function $g: \mathbf{U}_{1 / 50} \cap \mathbf{p}(\operatorname{spt} T) \rightarrow \mathbb{R}^{k}$ such that graph $g=\mathbf{C}_{1 / 50} \cap \Phi \circ \boldsymbol{\mu}_{7 / 8}(\operatorname{graph} \tilde{g}), g\left(y^{\prime}, \varphi_{0}\left(y^{\prime}\right)\right)=\left(\varphi_{1}\left(y^{\prime}\right), \ldots, \varphi_{k}\left(y^{\prime}\right)\right)$ for $\left(y^{\prime}, \varphi_{0}\left(y^{\prime}\right)\right) \in$ $\mathbf{C}_{1 / 50} \cap \mathbf{p}(\operatorname{spt} \partial T)$, and Lip $g \leq 2$.

From the excess decay Lemma 5.4 for $\tilde{T}$ we obtain the existence of a linear isometry, 0 of $\mathbb{R}^{n+k}$ with , $0=$ id on $\left(\mathbb{R}^{n-1} \times\{0\}\right) \times\{0\}^{k},\left\|,{ }_{0}-\mathrm{id}\right\| \leq C_{35} \epsilon_{1}$, and

$$
\mathbf{E}\left({ }_{0 \# \#}\left(\tilde{T}\left\llcorner\mathbf{C}_{1 / 2}\right), r\right) \leq C_{35}\left[r^{2 \beta} \mathbf{E}(\tilde{T}, 1)+\mathrm{K}(r)^{2}+\widehat{\mathrm{M}}(r)+\Omega(r)\right]\right.
$$

for $0<r<\frac{1}{3}$. Letting $\Psi=,{ }_{0}^{\circ} \boldsymbol{\mu}_{8 / 7} \circ \Phi \circ \boldsymbol{\mu}_{7 / 8} \circ,{ }_{0}^{-1}$ we have $\left(\Psi_{\#}, 0 \#\left(\tilde{T}\left\llcorner\mathbf{C}_{1 / 2}\right)\right)\left\llcorner\mathbf{C}_{1 / 3}=\right.\right.$ $\left(,{ }_{0 \#} \boldsymbol{\mu}_{8 / 7 \#}\left(T\left\llcorner\mathbf{C}_{7 / 16}\right)\right)\left\llcorner\mathbf{C}_{1 / 3}\right.\right.$ and

$$
\|D \Psi(\tilde{x})-\mathrm{id}\|=\left\|,{ }_{0} D \Phi\left(\frac{7}{8},{ }_{0}^{-1} \tilde{x}\right),{ }_{0}^{-1}-\mathrm{id}\right\|=\left\|D \Phi\left(\frac{7}{8},{ }_{0}^{-1} \tilde{x}\right)-\mathrm{id}\right\| \leq \kappa\left(\frac{7}{8}|\tilde{x}|\right) .
$$

On account of the smallness of $\left\|,{ }_{0}-\mathrm{id}\right\|$ and the controlled height of $\tilde{T}\left\llcorner\mathbf{C}_{1 / 2}\right.$ and $T\left\llcorner\mathbf{C}_{7 / 16}\right.$ we can therefore apply Lemma 6.3 to deduce with (6.23), the analog of (6.22), and $\kappa(r) \leq$ $\mathrm{K}(r)$, the estimate

$$
\mathbf{E}\left(,_{0 \#}\left(T\left\llcorner\mathbf{C}_{7 / 16}\right), r\right) \leq C_{36}\left[r^{2 \beta} \mathbf{E}(T, 1)+\mathrm{K}(r)^{2}+\widehat{\mathrm{M}}(r)+\Omega(r)\right]\right.
$$

for $0<r \leq \frac{1}{4}$.

Consider now $x \in \operatorname{spt} T$ with $\mathbf{p} x=y=\left(0, \ldots, 0, y_{n}\right)$ and $0<y_{n} \leq \frac{1}{8}$. For $\widehat{x}={ }_{0} x$ and $\widehat{y}=\mathbf{p} \widehat{x}$ we then have $\widehat{y}=\left(0, \ldots, 0, \widehat{y}_{n}\right)$ with $\frac{2}{3} y_{n} \leq \widehat{y}_{n} \leq \frac{3}{2} y_{n}$, because , ${ }_{0}=\mathrm{id}$ on $\left(\mathbb{R}^{n-1} \times\{0\}\right) \times\{0\}^{k},\left\|,{ }_{0}-\mathrm{id}\right\|$ is small, $T\left\llcorner\mathbf{C}_{r}\right.$ has height at most $r$ for $0<r \leq \frac{7}{16}$. Therefore $\mathbf{C}_{y_{n} / 2}(\widehat{y})$ is contained in $\mathbf{C}_{2 y_{n}}$, and from (6.24) we obtain

$$
\begin{aligned}
\mathbf{E}\left(,{ }_{0 \#}(T\right. & \left.\left\llcorner\mathbf{C}_{7 / 16}\right), \widehat{y}, \frac{1}{2} y_{n}\right) \\
& \leq 4^{n} C_{36}\left[\left(2 y_{n}\right)^{2 \beta} \mathbf{E}(T, 1)+\mathrm{K}\left(2 y_{n}\right)^{2}+\widehat{\mathrm{M}}\left(2 y_{n}\right)+\Omega\left(2 y_{n}\right)\right] .
\end{aligned}
$$

Since $\mathbf{C}_{y_{n} / 2}(\widehat{y})$ does not intersect the support of , $0 \#\left(\partial T\left\llcorner\mathbf{C}_{7 / 16}\right)\right.$, the hypotheses of the interior excess decay estimate in Lemma 5.4 are satisfied for $\left(\boldsymbol{\mu}_{y_{n} / 2}^{-1},{ }_{0} \boldsymbol{\tau}_{x}^{-1}\right)_{\#}\left(T\left\llcorner\mathbf{C}_{7 / 16}\right)\right)\llcorner\mathbf{C}$ and for the corresponding transformation of the integrand $F$. Consequently, there exists a linear isometry, ${ }_{x}$ of $\mathbb{R}^{n+k}$, close to the identity, such that for $T_{x}=\boldsymbol{\tau}_{x \#}^{-1}\left(T\left\llcorner\mathbf{C}_{7 / 16}\right)\right.$ we have

$$
\mathbf{E}\left({ }_{x \#} T_{x}, r\right) \leq C_{26}\left[\left(\frac{r}{y_{n}}\right)^{2 \beta} \mathbf{E}\left(,{ }_{0 \#}\left(T\left\llcorner\mathbf{C}_{7 / 16}\right), r\right)+\widehat{\mathrm{M}}(r)+\Omega(r)\right]\right.
$$

for $0<r \leq \frac{1}{6} y_{n}$. Combining this with (6.25), and using $\left(r / y_{n}\right)^{2 \beta} \Omega\left(2 y_{n}\right) \leq\left(r / y_{n}\right)^{2 \alpha} \Omega\left(2 y_{n}\right) \leq$ $2^{2 \alpha} \Omega(r) \leq 2^{2 \beta} \Omega(r)$ and the analogous inequality for $\widehat{\mathrm{M}}$ we infer

$$
\mathbf{E}\left(,{ }_{x \#} T_{x}, r\right) \leq C_{37}\left[r^{2 \beta} \mathbf{E}(T, 1)+\mathrm{K}(r)^{2}+\widehat{\mathrm{M}}(r)+\Omega(r)\right]
$$

for $x \in \operatorname{spt} T$ with $\mathbf{p} x=\left(0, \ldots, 0, y_{n}\right), 0<y_{n} \leq \frac{1}{8}$, and $0<r \leq \frac{1}{6} y_{n}$.

Now, choosing $\epsilon_{1}$ sufficiently small we obtain linear isometries $\Delta_{a}$ corresponding to $a \in$ $\mathbf{C}_{1 / 8} \cap \operatorname{spt} \partial T$ such that $\left\|\Delta_{a}-\mathrm{id}\right\| \leq \operatorname{const}(n, k) \kappa(|a|)$ and the scaled currents $\left(\left(\boldsymbol{\mu}_{4} \Delta_{a} \boldsymbol{\tau}_{a}^{-1}\right)_{\#}\right.$ 
$\left(T\left\llcorner\mathbf{C}_{7 / 16}\right)\right)\llcorner\mathbf{C}$ together with the correspondingly scaled version of $F$ satisfy all the assumptions which we have used for $T$ above to derive (6.24) and (6.26). Here, the smallness of the excess follows from (6.17) and Lemma 5.3. More precisely, with a mass estimate as in (6.22) we obtain the following estimate, valid for $a \in \mathbf{C}_{1 / 8} \cap \operatorname{spt} \partial T, T_{a}=\boldsymbol{\tau}_{a \#}^{-1}\left(T\left\llcorner\mathbf{C}_{7 / 16}\right)\right.$, some linear isometry, ${ }_{a}$ of $\mathbb{R}^{n+k}$ close to the identity, and $0<r \leq \frac{1}{16}$ :

$$
\begin{aligned}
\mathbf{E}\left(,{ }_{a \#} T_{a}, r\right) & \leq C_{38}\left[r^{2 \beta}\left(\mathbf{E}(T, 1)+\kappa(|a|)^{2}\right)+\mathrm{K}(r)^{2}+\widehat{\mathrm{M}}(r)+\Omega(r)\right] \\
& \leq C_{39}\left[r^{2 \beta} \mathbf{E}(T, 1)+\mathrm{K}(r)^{2}+\widehat{\mathrm{M}}(r)+\Omega(r)\right] .
\end{aligned}
$$

Here we have used $r^{\beta} \kappa(|a|) \leq r^{\alpha} \kappa(1) \leq \kappa(r) \leq \mathrm{K}(r)$. Moreover, if $b \in \mathbf{C}_{1 / 32} \cap$ spt $T \backslash \operatorname{spt} \partial T$ and $a$ is a nearest point to $b$ in spt $\partial T$, then $|a|<\frac{1}{16}$ and $\mathbf{p} \boldsymbol{\mu}_{4} \Delta_{a} \boldsymbol{\tau}_{a}^{-1}(b)=\left(0, \ldots, 0, y_{n}\right)$ with $0<y_{n} \leq 4|b-a|<\frac{1}{8}$. Hence (6.26) implies, by the same reasoning, the existence of a linear isometry, ${ }_{b}$ of $\mathbb{R}^{n+k}$ close to the identity such that $T_{b}=\boldsymbol{\tau}_{b \#}^{-1}\left(T\left\llcorner\mathbf{C}_{7 / 16}\right)\right.$ satisfies

$$
\mathbf{E}\left(,{ }_{b \#} T_{b}, r\right) \leq C_{40}\left[r^{2 \beta} \mathbf{E}(T, 1)+\mathrm{K}(r)^{2}+\widehat{\mathrm{M}}(r)+\Omega(r)\right]
$$

for $0<r \leq \frac{1}{24} y_{n}$. Since $4|b-a| \leq \sqrt{2} y_{n}$, because $\boldsymbol{\mu}_{4 \#} \Delta_{a \#} \boldsymbol{\tau}_{a \#}^{-1}\left(T\left\llcorner\mathbf{C}_{7 / 16}\right)\right.$ has height in $\mathbf{C}_{r}$ not exceeding $r$ for $0<r \leq \frac{7}{16}$, we see that (6.28) is valid in particular for $b \in$ $\mathbf{C}_{1 / 32} \cap \operatorname{spt} T \backslash \operatorname{spt}(\partial T)$ and $0<r \leq \frac{1}{9} \operatorname{dist}(\mathbf{p} b, \mathbf{p}(\operatorname{spt}(\partial T))$.

With (6.27) and (6.28) we can now proceed exactly as in Step 4 of the proof of Theorem 6.1 in order to establish the asserted continuity modulus (6.18) for $D g$. One uses the fact that $T\left\llcorner\mathbf{C}_{1 / 50}\right.$ is represented by the graph of $g$ with $\operatorname{Lip} g \leq 2$ and estimates $|D g(a)-D g(b)|^{2}$ for $a, b \in \mathbf{C}_{1 / 50} \cap \mathbf{p}(\operatorname{spt} T)$ distinguishing the three special cases $a$, $b \in \mathbf{p}(\operatorname{spt} \partial T)$, resp. $a \in \mathbf{p}(\operatorname{spt} \partial T)$ and $b \notin \mathbf{p}(\operatorname{spt} \partial T)$, resp. $a, b \notin \mathbf{p}($ spt $\partial T)$ with $|a-b| \leq \frac{1}{9} \operatorname{dist}(a, \mathbf{p}(\operatorname{spt} \partial T)) \leq \frac{1}{9} \operatorname{dist}(b, \mathbf{p}(\operatorname{spt} \partial T))$. The general case is reduced to the special cases by the triangle inequality. As the reasoning is clear from the proof of Theorem 6.1 we can omit details.

\section{Proof of the boundary regularity theorem}

For completeness we indicate here how the boundary regularity theorem stated in the introduction follows from the $\epsilon$-regularity theorem. The proof follows [Ha1, Section 3] closely, with simple modifications to treat almost minimizing currents. We first observe that the following compactness lemma for almost minimizing currents holds:

7.1 Lemma (compactness for sequences of almost minimizing currents). Suppose $T_{i} \in \mathcal{R}_{n}\left(\mathbb{R}^{n+k}\right)$ is $\left(F_{i}, \omega_{i}\right)$ minimizing in the open subset $D$ of $\mathbb{R}^{n+k}$ for $i=1,2, \ldots$, where the parametric integrands $F_{i}$ satify (1.1) uniformly and converge to $F$ uniformly on compact subsets of $D \times \bigwedge_{n} \mathbb{R}^{n+k}$ as $i \rightarrow \infty$ and where $\limsup _{i \rightarrow \infty} \omega_{i} \leq \omega$ holds on $] 0, \infty$ [ with a nondecreasing function $\omega:] 0, \infty[\rightarrow[0, \infty]$ which is continuous from the right with $\omega(0+)=$ 0 . If $T_{i} \rightarrow T_{0}$ holds locally on $D$ with respect to the flat metric distance and $\sup _{i} \mathbf{M}\left(T_{i}\right)<\infty$ then $T_{0} \in \mathcal{R}_{n}\left(\mathbb{R}^{n+k}\right)$ is $(F, \omega)$ minimizing in $D$. Moreover, every subset $A$ of $D$ with positive distance to $\left(\operatorname{spt} T_{0}\right) \cup \bigcup_{i=1}^{\infty}$ spt $\partial T_{i}$ intersects only finitely many of the sets $\operatorname{spt} T_{i}$.

The proof is a straightforward adaptation of [Ha1, Lemma 3.5] which in turn is modelled after [Fe, 5.4.2]. It uses only the definition of almost minimality, standard properties of 
convergence with respect to the flat distance [Fe, 4.1.24], and the lower mass bound from Lemma 2.1 which holds uniformly for the $T_{i}$ with sufficiently large $i$ by the hypotheses on the integrands $F_{i}$ and the functions $\omega_{i}$. (Note that $\omega_{i}(r) \leq 1+\omega(r)<\infty$ for some $r>0$ and all sufficiently large $i$.) We omit the details and proceed to the

Proof of the boundary regularity theorem. We argue by contradiction verifying also the assertions made in Section 0 about the dependence of the constant $\vartheta>0$ on the data. If the theorem were false we would have, after applying linear isometries to transform the point $a$ to the origin and the tangent space of the boundaries at $a$ to $\mathbb{R}^{n-1} \times$ $\{0\}^{k+1} \subset \mathbb{R}^{n+k}$ and after suitable scalings by homotheties of $\mathbb{R}^{n+k}$, sequences of currents $T_{i} \in \mathcal{R}_{n}\left(\mathbb{R}^{n+k}\right)$, parametric integrands $F_{i}$, and $C^{1, \beta}$ functions $\varphi_{i}: \mathbf{U}^{n-1}(0,1) \rightarrow \mathbb{R}^{1+k}$, such that no $T_{i}$ satisfies the conclusion of Theorem 6.4 locally at 0 and the following assertions hold:

$$
\begin{aligned}
& T_{i} \text { is }\left(F_{i}, \omega_{i}\right) \text { minimizing in } \mathbf{U}(0,1)=\mathbf{U}^{n+k}(0,1) \text { with } \omega_{i}(r)=\sigma_{i} r^{\beta} \\
& \lim _{i \rightarrow \infty} \sigma_{i}=0
\end{aligned}
$$

$\left(\partial T_{i}\right)\left\llcorner\mathbf{U}(0,1)\right.$ is represented by the graph of $\varphi_{i}$ taken with suitable orientation and multiplicity 1 ; morover $\varphi(0)=0, D \varphi(0)=0$, and

$$
\left\|D \varphi\left(y^{\prime}\right)-D \varphi\left(y^{\prime \prime}\right)\right\| \leq \kappa_{i}\left|y^{\prime}-y^{\prime \prime}\right|^{\beta} \quad \text { for } y^{\prime}, y^{\prime \prime} \in \mathbf{U}^{n-1},
$$

with $\lim _{i \rightarrow \infty} \kappa_{i}=0$;

$\mathbf{M}\left(T_{i}\llcorner\mathbf{U}(0,1)) \leq \frac{1}{2} \boldsymbol{\alpha}(n)+\frac{1}{i} ;\right.$

$F_{i}$ satisfies the structure conditions (1.1)-(1.12) with $\lim _{i \rightarrow \infty} \mu_{i}=0$

and with $\Lambda, \lambda, \nu(\bullet)$ independent of $i$.

Moreover, as the $F_{i}$ satisfy a uniform Lipschitz condition on each compact subset of $\mathbf{U}(0,1) \times$ $\bigwedge_{n} \mathbb{R}^{n+k}$ we may assume, after passing to a subsequence, that for some continuous integrand $F_{0}: \mathbf{U}(0,1) \times \bigwedge_{n} \mathbb{R}^{n+k} \rightarrow\left[0, \infty\left[\right.\right.$ (which has constant coefficients, because $\mu_{i} \rightarrow 0$ ) we have

$$
F_{i} \rightarrow F_{0} \text { uniformly on compact subsets of } \mathbf{U}(0,1) \times \bigwedge_{n} \mathbb{R}^{n+k} \text { as } i \rightarrow \infty \text {. }
$$

Now, since $\mathbf{M}\left(T_{i}\llcorner\mathbf{U}(0,1))+\mathbf{M}\left(\left(\partial T_{i}\right)\llcorner\mathbf{U}(0,1))\right.\right.$ is bounded, by (7.3) and (7.2), we may apply the local version of the compactness theorem [Si1, 31.3] (cf. the reasoning in the proof of [Fe, 5.4.3]) to deduce, passing to a subsequence again, convergence $T_{i} \rightarrow T_{0} \in \mathcal{R}_{n}\left(\mathbb{R}^{n+k}\right)$ locally on $\mathbf{U}(0,1)$ in the flat metric distance. Clearly,

$$
\mathbf{M}\left(T_{0}\right) \leq \frac{1}{2} \boldsymbol{\alpha}(n)
$$

holds, by (7.3), and

$$
\mathbf{U}(0,1) \cap \operatorname{spt}\left(\partial T_{0}\right)=\mathbf{U}^{n-1}(0,1) \times\{0\}^{1+k} \subset \mathbb{R}^{n+k}
$$

by (7.2). Using the nonexpanding map $f(y, z)=\left(y_{1}, \ldots, y_{n-1},\left|\left(y_{n}, z\right)\right|\right)$ from $\mathbb{R}^{n} \times \mathbb{R}^{k}$ to $\mathbb{R}^{n-1} \times[0, \infty[$ one gets from $(7.6)$

$$
\mathbf{M}\left(f_{\#} T_{0}\right) \leq \int_{\mathbf{U}(0,1)}\left|\left(\bigwedge_{n} D f\right) \vec{T}_{0}\right| d\left\|T_{0}\right\| \leq \int_{\mathbf{U}(0,1)} d\left\|T_{0}\right\| \leq \frac{1}{2} \boldsymbol{\alpha}(n),
$$


and since, for $\mathbf{U}=\mathbf{U}^{n}(0,1)$,

$$
\mathbf{U} \cap \operatorname{spt}\left(f_{\#} T_{0}\right) \subset f\left(\mathbf{U}^{n-1} \times\{0\}^{1+k}\right)=\mathbf{U}^{n-1} \times\{0\} \subset \mathbf{U}
$$

by (7.7) and the definition of $f$, one deduces with the constancy theorem [Fe, 4.1.7] that $f_{\#} T_{0}$ represents the half ball $\mathbf{U}_{+}$with multiplicity +1 or -1 and that $\left|\left(\bigwedge_{n} D f\right) \vec{T}_{0}\right|=1$ holds $\left\|T_{0}\right\|$-almost everywhere. This means that $\vec{T}_{0}(y, z)$ is an orientation vector of the $n$ dimensional subspace $\mathbb{R}(y, z)+\mathbb{R}^{n-1} \times\{0\}$ of $\mathbb{R}^{n+k}$ for $\left\|T_{0}\right\|$-almost all $(y, z) \in \mathbb{R}^{n} \times\left(\mathbb{R}^{k} \backslash\{0\}\right)$, and slicing with the map $(y, z) \mapsto\left|\left(y_{n}, z\right)\right|^{-1}\left(y_{n}, z\right) \in S^{k}$ one sees, using also (7.6), that $T_{0}$ represents an $n$-dimensional half ball in $\mathbb{R}^{n+k}$ with equator $\mathbf{U}^{n-1} \times\{0\}$. (See [Br2, 4.5] where the argument of [Ha2, Corollary 1] is adapted to the present situation.) After a suitable orthogonal transformation of $\mathbb{R}^{n+k}$ we may assume $T_{0}=\left(\mathbf{E}^{n}\left\llcorner\mathbf{U}_{+}\right) \times \boldsymbol{\delta}_{0}^{k}\right.$.

By Lemma 7.1, $T_{0}$ is absolutely minimizing for $F_{0}$, and for every $0<\delta<1$ the set $\{(y, z) \in \mathbf{B}(0,1-\delta):|z| \geq \delta\}$ intersects only finitely many of the sets $\operatorname{spt} T_{i}$. It is now clear from (7.1)-(7.4) and the constancy theorem [Fe, 4.1.7] that the currents

$$
\tilde{T}_{i}=\left(\boldsymbol{\mu}_{1-\delta}^{-1}\right)_{\#}\left(T_{i}\left\llcorner\mathbf{U}^{n}(0,1-\delta) \times \mathbf{U}^{k}(0, \delta)\right) \in \mathcal{R}_{n}(\mathbf{C})\right.
$$

for $\delta>0$ fixed sufficiently small and for $i$ sufficiently large satisfy the hypotheses of the boundary case in Theorem 6.4 for the integrands $\tilde{F}_{i}$ and functions $\tilde{\omega}_{i}$ obtained from $F_{i}$ and $\omega_{i}$ by scaling with $\boldsymbol{\mu}_{1-\delta}^{-1}$. Therefore, for $i$ sufficiently large the currents $\tilde{T}_{i}$ are regular at the origin as desribed in the conclusion of Theorem 6.4. This contradicts, however, our assumption on the sequence $T_{i}$.

In the interior situation $a \in \operatorname{spt} T \backslash \operatorname{spt}(\partial T)$ one can conclude with a similar reasoning that the $(F, \omega)$ minimizing current $T$ is regular locally at $a$, provided the $n$-dimensional density of $\|T\|$ has a local minimum at $a$ (on the complement of a set of $\|T\|$ measure zero) and Tan $(\operatorname{spt} T, a)$ is an $n$-dimensional subspace of $\mathbb{R}^{n+k}$ (cf. [Fe, 5.3.16] and [Bo, VIII]). The interior case of Theorem 6.4 then gives the same modulus of continuity for the field of the tangent $n$-planes to spt $T$ near $a$ as in our boundary regularity theorem.

\section{References}

[All1] Allard, W.K.: On the first variation of a varifold. Annals of Math. 95 (1972), 417-491.

[All2] Allard, W.K.: On the first variation of a varifold: boundary behaviour. Annals of Math. 101 (1975), $418-446$.

[Alm1] Almgren, F. J.: Existence and regularity almost everywhere of solutions to elliptic variational problems among surfaces of varying topological type and singularity structure. Annals of Math. 87 (1968), 321-391.

[Alm2] Almgren, F. J.: Existence and regularity almost everywhere of solutions to elliptic variational problems with constraints. Mem. Am. Math. Soc. 165 (1976).

[Alm3] Almgren, F. J.: Q-valued functions minimizing Dirichlet's integral and the regularity of area minimizing rectifiable currents up to codimension two. Princeton Univ. 1984, 3745 g; see also Bull. Amer. Math. Soc.8 (1983), 327-328.

[Alm4] Almgren, F. J.: Optimal isoperimetric inequalities. Indiana Univ. Math. J. 35 (1986), 451-547.

[Bo] Bombieri, E.: Regularity theory for almost minimal currents. Arch. Rational Mech. Anal. 7 (1982), $99-130$. 
[Br1] Brothers, J. E.: The structure of solutions to Plateau's problem in the $n$-sphere. J. Diff. Geometry 11 (1976), 387-400.

[Br2] Brothers, J. E.: Existence and structure of tangent cones at the boundary of an area minimizing integral current. Indiana Univ. Math. J. 26 (1977), 1027-1044.

[Ca] Campanato, S.: Equazioni ellittiche del $\mathrm{II}^{e}$ ordine e spazi $\mathcal{L}^{2, \lambda}$. Ann. Mat. Pura Appl. 69 (1965), 321-380.

[DeG] DeGiorgi, E.: Frontiere orientate di misura minima. Sem. Mat. Norm. Sup. Pisa, 1960-61.

[Du1] Duzaar, F.: On the existence of surfaces with prescribed mean curvature and boundary in higher dimensions. Analyse non linéaire 10 (1993), 191-214.

[Du2] Duzaar, F.: Boundary regularity for area minimizing currents with prescribed volume. J. of Geom. Anal. 7 (1997), 585-592.

[DF1] Duzaar, F., Fuchs, M.: On the existence of integral currents with prescribed mean curvature vector. Manuscripta math. 67 (1990), 41-67.

[DF2] Duzaar, F., Fuchs, M.: A general existence theorem for integral currents with prescribed mean curvature form. Bolletino U.M.I. (7) 6-B (1992), 901-912.

[DGa] Duzaar, F., Gastel, A.: Nonlinear elliptic systems with Dini continuous coefficients. Preprint SFB 288, Berlin.

[DGr] Duzaar, F., Grotowski, J.F.: Partial regularity for nonlinear elliptic systems: The method of $A$ harmonic approximation. To appear in Manuscripta. Math.

[DGG] Duzaar, F., Gastel, A., Grotowski, J.F.: Partial regularity for almost minimizing functions of quasiconvex integrands. Preprint SFB 288, Berlin.

[DS1] Duzaar, F., Steffen, K.: $\lambda$ minimizing currents. Manuscripta math. 80 (1993), 403-447.

[DS2] Duzaar, F., Steffen, K.: Boundary regularity for minimizing currents with prescribed mean curvature. Calc. Var. 1 (1993), 355-406.

[DS3] Duzaar, F., Steffen, K.: Existence of hypersurfaces with prescribed mean curvature in Riemannian manifolds. Indiana Univ. Math. J. 45 (1996), 1045-1093.

[Fe] Federer, H.: Geometric measure theory. Springer-Verlag, Berlin Heidelberg New York, 1969.

[Fe1] Federer, H.: The singular set of area minimizing rectifiable currents with codimension one and of area minimizing flat chains modulo two with arbitrary codimension. Bull. Amer. Math. Soc. 76 (1970), $767-771$.

[Gi] Giaquinta, M.: Multiple Integrals in the Calculus of Variations and Nonlinear Elliptic Systems. Princeton NJ, Princeton University Press, 1983.

[GMT] Gonzalez, E., Massari, U., Tamanini, I.: On the regularity of boundaries of sets minimizing perimeter with a volume constraint. Indiana Univ. Math. J. 32 (1983), 25-37.

[Ha1] Hardt, R.: On boundary regularity for integral currents or flat chains modulo two minimizing the integral of an elliptic integrand. Comm. Partial Differ. Equations 2 (1977), 1163-1232.

[Ha2] Hardt, R.: Uniqueness of nonparametric area minimizing currents. Indiana Univ. Math. J. 26 (1977), $65-71$.

[HaL] Hardt, R., Lin, F.-H.: Tangential regularity near the $C^{1}$ boundary. Proc. Symposia Pure Math. 44 (1986), 245-253.

[HS] Hardt, R., Simon, L.: Boundary regularity and embedded solutions for the oriented Plateau problem. Annals of Math. 110 (1979), 439-486.

[Li] Lin, F.-H.: Regularity for a class of parametric obstacle problems. Thesis, Univ. of Minnesota, 1985.

[Ma] Massari, U.: Esistenza e regolarità delle ipersuperfici di curvatura media assegnata in $\mathbb{R}^{n}$. Arch. Rational Mech. Anal. 55 (1974), 357-382. 
[MM] Massari, U., Miranda, M.: Minimal surfaces of codimension one. Mathematical studies, vol. 91, North-Holland, Amsterdam 1984.

[Mi] Miranda, M.: Frontiere orientate con ostacoli. Ann. Univ. Ferrara 16 (1971), 29-37.

[Mo] Morrey, C.B.: Multiple integrals in the calculus of variations. Springer-Verlag, Berlin Heidelberg New York, 1966.

[SS] Schoen, R., Simon, L.: A new proof of the regularity for rectifiable currents which minimize parametric elliptic functionals. Indiana Univ. Math. J. 31 (1982), 413-434.

[SSA] Schoen, R., Simon, L., Almgren, F.J.: Regularity and singularity estimates on hypersurfaces minimizing parametric elliptic variational integrals. Acta Math. 139 (1977), 217-265.

[Si1] Simon, L.: Lectures on geometric measure theory. Proc. CMA, Vol. 3, ANU Canberra, 1983.

[Si2] Simon, L.: Theorems on regularity and singularity of energy minimizing maps. Birkhäuser-Verlag, Basel Boston Berlin, 1996.

[Sv1] Šverak, V.: Quasiconvex functions with subquadratic growth. Proc. Roy. Soc. London A 433 (1991), $223-225$.

[Sv2] Šverak, V.: Rank-one convexity does not imply quasiconvexity. Proc. Roy. Soc. Edinburgh 120 (1992), 185-189.

[Ta1] Tamanini, I.: Regularity results for almost minimal hypersurfaces in $\mathbb{R}^{N}$. Quaderni Dip. Mat. Univ. Lecce, Q1 (1984).

[Ta2] Tamanini, I.: Boundaries of Cacciopoli sets with Hölder-continuous normal vector. J. reine Angew. Math. 334 (1982), 27-39.

[Ta3] Tamanini, I.: Variational problems of least area type with constraints. Ann. Univ. Ferrara 34 (1988), $183-217$.

Mathematisches Institut der Humboldt-Universität zu Berlin, Unter den Linden 6, D-10099 Berlin, Germany

and

Mathematisches Institut der Heinrich-Heine-Universität Düsseldorf Universitätstraße 1, D-40225 Düsseldorf, Germany. 